\title{
Continuous-Time Quantum Walks: Models for Coherent Transport on Complex Networks
}

\author{
Oliver Mülken and Alexander Blumen \\ Theoretical Polymer Physics, University of Freiburg, Hermann-Herder-Straße 3, 79104 Freiburg, Germany
}

(Dated: June 2, 2018)

\begin{abstract}
This paper reviews recent advances in continuous-time quantum walks (CTQW) and their application to transport in various systems. The introduction gives a brief survey of the historical background of CTQW. After a short outline of the theoretical ideas behind CTQW and of its relation to classical continuous-time random walks (CTRW) in Sec. 2, implications for the efficiency of the transport are presented in Sec. 3. The fourth section gives an overview of different types of networks on which CTQW have been studied so far. Extensions of CTQW to systems with long-range interactions and with static disorder are discussed in section V. Systems with traps, i.e., systems in which the walker's probability to remain inside the system is not conserved, are presented in section IV. Relations to similar approaches to the transport are studied in section VII. The paper closes with an outlook on possible future directions.
\end{abstract}

PACS numbers: $05.60 . \mathrm{Gg}, 05.60 . \mathrm{Cd}, 71.35 .-\mathrm{y}$

\section{Contents}

\section{Introduction}
A. Quantum Walks
B. Experimental implementations of CTQW
C. Transport in complex systems

\section{Definitions and terminology of CTQW}
A. Connectivity of network
B. Transfer matrix for CTRW
C. Hamiltonian for CTQW
D. Example: A discrete ring

\section{Efficiency of CTQW and CTRW}
A. Examples

\section{CTQW on networks}

A. Deterministic networks

1. Two dimensional regular networks

2. Star-like networks

3. Complete graph

4. Dendrimers

5. Husimi-cacti

6. Glued Cayley trees

B. Fractals

1. Sierpinski Gaskets

C. Statistical networks

1. Small-world networks

2. Erdös-Rényi networks

3. Scale-free networks

4. Apollonian networks

\section{Extensions}
A. Systems with long-range interactions
B. Systems with disorder and localization

\section{Systems with absorption}
A. Average survival probability
B. Regular networks

1. Ring with traps
2. Line with traps

3. Line with traps and long-range interactions

C. Fractals

1. Hyperbranched fractals

D. Random networks

1. Disordered system with one trap

VII. Relations between CTRW/CTQW and other approaches

A. Phase space approaches

1. WF for a ring

2. Rings with energetic disorder

3. Long-range interaction cycles

4. Disordered networks

B. Quantum master equations

1. Decoherence on rings

2. Dimer with traps

VIII. Outlook

Acknowledgements

References

\section{INTRODUCTION}

Transport of mass, charge or energy is the basis of many physical, chemical or biological processes. Such transfer mechanisms and their efficiency depend on the underlying structure of the system, range from polymer physics to solid state physics to biological physics to even quantum computation, see Refs. [1-6] for reviews. The underlying structures could be, for example, simple crystals, as in solid state physics [7], more complex molecular aggregates like polymers [8], or general network structures [2].

In quantum mechanics, the potential a particle is moving in specifies the Hamiltonian of the system, which determines the time evolution. For instance, the dynamics of electrons in a simple crystal is described by the Bloch ansatz [7, 9, 10], 
which mirrors their behavior in metals quite accurately. Over the years these models have been refined and extended to address different phenomena, such as the dynamics of atoms in optical lattices and the Anderson localization in systems with energetic disorder [11]. In quantum chemistry, Hückel's molecular-orbital theory allows to define a Hamiltonian for more complex structures, such as molecules [12]. This is also related to transport in polymers, where the connectivity of the polymer plays a fundamental role in its dynamical and relaxational properties [13]. There, (classical) transport processes can be modeled by continuous-time random walk (CTRW) approaches [5, 6, 8]. An important example is the motion of Frenkel excitons, whose high-temperature dynamics is often governed by a master equation with an appropriate (classical) transfer operator which determines the temporal evolution of the system [14, 15]. CTRW have proven to be a very useful and general tool in describing incoherent transport in various settings, ranging, for instance, from percolation on fractals [16] to electronic energy transfer in glassy systems [17, 18]. Also, anomalous diffusional behavior has been successfully modeled by CTRW using suitable waiting time distributions [19, 20].

\section{A. Quantum Walks}

The tight-binding approximation used in sold state physics as well as in Hückel's theory is equivalent to the so-called quantum walks, which model purely coherent quantum dynamics of excitations on networks [4, 21-24]. What matters is that the constituting elements (spins, atoms, molecules, etc.) are of the same type, in the simplest cases acting as twolevel systems. Now, the extension of classical random walks ideas to the quantum domain is not unique and allows variants, of which two types capture most of the interest: discretetime quantum (random) walks (introduced by Aharonov et al., using an additional internal "coin" degree of freedom [22]), and continuous-time quantum walks (CTQW) (introduced by Farhi and Gutmann, where the connection to CTRW uses the analogy between the quantum mechanical Hamiltonian and the classical transfer matrix [21]). Recently, Strauch has shown how these two versions are related [25]. Thus, CTQW and CTRW act as the two extreme cases of purely coherent and purely incoherent transport, respectively.

In quantum information theory, quantum walks are used extensively as algorithmic tools for quantum computation [26]. Here, the most prominent examples are Shor's algorithm [27] and Grover's algorithm [28]. The latter is a quantum algorithm for finding an item in an (unsorted) database of qubits. Such an algorithm can also be related to CTQW [29, 30]. The idea of using computers built on quantum mechanical principles instead of classical computers goes back to Feynman, who already formulated an early version of quantum walks in 1982 [31]. From a conceptual point of view, quantum walks are closely related to quantum cellular automata, see, e.g., [32]. Using CTQW, the transfer of information in complex systems was discussed by Christandl et al. in the context of perfect state transfer [33].
CTQW are also in close relation to the so-called quantum graphs (QG), see, e.g., [34-37]. However, unlike CTQW, QG take into account detailed properties of each bond of the graph explicitly; in QG, bonds may be directed and have distinct lengths. The connections between discrete Laplacians on discrete QG and periodic orbits were recently investigated by Smilansky [38].

\section{B. Experimental implementations of CTQW}

Simple theoretical models have always been very useful for our understanding of physics. In quantum mechanics, next to the harmonic oscillator, the particle in a box provides much insight into the quantum world (e.g. [39]). The problem of a quantum mechanical particle moving in an infinite box has been reexamined in [40-42]: Remarkably, even this simple system shows complex but regular spacetime probability structures (so-called quantum carpets). In solid state physics and quantum information theory, one of the most simple systems is associated with a particle moving in a regular periodic potential.

In recent years, arrangements close to ideal theoretical models have been tailored using, e.g., ultra-cold atoms in optical lattices, see [43] and references therein. For both quantum walk variants, experimental implementations have been proposed, based on microwave cavities [44], on Rydberg atoms [45] or on ground state atoms [46] in optical lattices or in optical cavities [47], or using the orbital angular momentum of photons [48]. Other experimental proposals connected to CTQW are based on waveguide arrays [49] or on structured clouds of Rydberg atoms [50].

\section{Transport in complex systems}

The range of systems where CTQW can be used to study transport is clearly not restricted to simple (symmetric) models. In particular, CTQW have been proven to be very useful in describing the dynamics of excitations in various complex systems, as will be shown throughout this review.

Since CTQW can be related to the tight-binding approximation in solid state physics, CTQW are also related to chains of coupled spin $1 / 2$ particles. If all but one spins are prepared in the same state, say, spin down, and a single spin in the spin up state, one can map this onto a chain of coupled two-level systems with a single initial excitation at the node which is identified with the spin up particle [24, 51, 52]. The essential dynamics is then equivalent to a CTQW on a linear network.

However, also the dynamics in topological disordered systems can be modeled by CTQW. Take for instance a gas of highly excited ultra-cold Rydberg atoms. At ultra-low temperatures the configuration of the atoms can be thought of as being frozen on the time-scale on which the following transport process is taking place [53-55]: The dynamics is started by exciting one (or several) of the Rydberg atoms in a higher state which is resonantly coupled to a lower Rydberg state [45, 50]. Since essentially only two different Rydberg states 
are involved, one can map this again onto a network of coupled two-level systems.

Also biological systems share some properties of simple network models. Recent experiments on the light-harvesting complexes of algae have renewed the interest in studying the dynamics of excitations in such systems [56, 57]. Here, an initial excitation (a Frenkel exciton) is created by absorbing a solar photon. The exciton is then transfered along a network of (bacterio-) chlorophylls (BChl), the chromophores, to the reaction center, where the excitation energy is converted into chemical energy. Now, the network of the BChls can be considered to be static and stable with defined couplings between the BChls, at all (even ambient) temperatures of interest. Moreover, the Frenkel exciton can be viewed as a quasi particle moving along the network. Therefore, also here the transport can be modeled by CTQW, at least at (very) low temperatures. The incoherent exciton transport in dendrimers can be efficiently modelled by random walks, see, for instance, [58-61]. However, for certain systems there is also experimental evidence for coherent interchromophore transport processes [62,63]. Recent investigations of biological light-harvesting systems range from the Fenna-MatthewsOlsen complex [56] to marine algae [57]. There have also been recent theoretical efforts to understand the coherent features of exciton dynamics found in recent experiments, see, e.g., [64-68].

Clearly, such biological systems cannot be thought of as being isolated from their environment. Therefore, in these cases the purely coherent description by CTQW is of limited value. However, it is also possible to extend the CTQW approach to take the coupling to the environment into account, which leads to models related to quantum master equations [69], see also Sec.VIIB

\section{DEFINITIONS AND TERMINOLOGY OF CTQW}

\section{A. Connectivity of network}

Networks involved in the CTQW and CTRW dynamics are characterized by the form in which their sites are connected. Starting point of a network is a collection of $N$ nodes, which are then joined by bonds, the connectivity matrix $\boldsymbol{A}$ mirroring the way in which the bonding occurs.

The $N \times N$ connectivity matrix $\boldsymbol{A}$ has the elements $A_{k j}$, where

$$
A_{k j}= \begin{cases}f_{j} & \text { for } k=j \\ -1 & \text { if } k \text { and } j \text { connected } A_{k j}=A_{j k} \\ 0 & \text { else }\end{cases}
$$

where $f_{j}$ is the number of bonds emanating from node $j$. This matrix has interesting and useful properties: (a) $\boldsymbol{A}$ is real and symmetric, (b) all its eigenvalues $\lambda_{n}$ are real and $\lambda_{n} \geq 0$, and (c) $\boldsymbol{A}$ has a single smallest eigenvalue which is $\lambda_{1}=0$.

One can associate with every node of the network a basis vector in an $N$-dimensional vector space. Now, these basis vectors form a complete orthonormal basis, which, for in- stance, is given by

$$
|1\rangle=\left(\begin{array}{c}
1 \\
0 \\
\vdots \\
0
\end{array}\right), \quad|2\rangle=\left(\begin{array}{c}
0 \\
1 \\
\vdots \\
0
\end{array}\right), \quad \ldots,|N\rangle=\left(\begin{array}{c}
0 \\
\vdots \\
0 \\
1
\end{array}\right)
$$

Take as an example a ring-like network of $N$ nodes, with only nearest neighbor connections and $|N+1\rangle \equiv|1\rangle$, then the matrix $\boldsymbol{A}$ reads:

$$
A_{i j}=\left(\begin{array}{cccccc}
2 & -1 & 0 & \ldots & & -1 \\
-1 & 2 & -1 & 0 & \ldots & 0 \\
0 & \ddots & \ddots & \ddots & \ddots & \vdots \\
\vdots & & & & & \\
& & & -1 & 2 & -1 \\
-1 & 0 & \ldots & & -1 & 2
\end{array}\right)
$$

Writing $\boldsymbol{A}$ in a quantum mechanical fashion using the projection operators $|l\rangle\langle k|$, leads to

$$
\boldsymbol{A}=\sum_{l} 2|l\rangle\langle l|-| l-1\rangle\langle l|-| l+1\rangle\langle l|
$$

Having now specified the network through the connectivity matrix $\boldsymbol{A}$, one is interested in the dynamics over the network. First, one distinguishes between purely classical, i.e., incoherent dynamics and purely quantum-mechanical, i.e., coherent dynamics. The main question one wishes to answer is: What is the probability to be at node $k$ after time $t$ when starting at node $j$ ? While here the focus is mainly on localized initial conditions at a single node $j$, in general, the initial state can be any distribution involving all the nodes.

\section{B. Transfer matrix for CTRW}

We start by considering classical transport. Let the initial node be $j$, such that the initial state of the system is $|j\rangle$, and denote the transition probability to go in time $t$ from node $j$ to node $k$ by $p_{k, j}(t)$. Therefore, the initial condition is $\langle k \mid j\rangle \equiv$ $p_{k, j}(0)=\delta_{k, j}$, where $\delta_{k, j}=1$ for $k=j$ and 0 otherwise. The state after time $t$ is $|j ; t\rangle$, such that the overlap with node $k$ reads $\langle k \mid j ; t\rangle \equiv p_{k, j}(t)$.

The dynamics resulting in the state $|j ; t\rangle$ follows from the transition rates per unit time between two nodes. Those transition rates are the elements of the so-called transfer matrix $\boldsymbol{T}, T_{k j} \equiv\langle k|\boldsymbol{T}| j\rangle$, which is therefore related to the spatial gain or loss. One can now write the temporal change of the probability after time $\epsilon \ll 1$ as

$$
p_{k, j}(\epsilon)=p_{k, j}(0)+\epsilon T_{k j}=\delta_{k, j}+\epsilon T_{k j} .
$$

Assuming a Markovian process, the following master equation can be shown to hold [5, 6]:

$$
\frac{d}{d t} p_{k, j}(t)=\sum_{l} T_{k l} p_{l, j}(t)
$$


This equation defines the CTRW, which (depending on the specific form of the transfer matrix) have been applied to various problems in physics, chemistry, biology, and also social sciences.

In the simplest case, where the rates for all bonds are equal, say, $\gamma$, the transfer matrix is related to the connectivity matrix through $\boldsymbol{T}=-\gamma \boldsymbol{A}$. Then the master equation describes a diffusive motion over the network.

The formal solution of Eq.(6) is $p_{k, j}(t)=\left\langle k\left|e^{\boldsymbol{T} t}\right| j\right\rangle=$ $\left\langle k\left|e^{-\gamma \boldsymbol{A} t}\right| j\right\rangle$. Denoting the eigenstates of $\boldsymbol{A}$ by $\left|q_{n}\right\rangle$, one has

$$
p_{k, j}(t)=\left\langle k\left|e^{-\gamma \boldsymbol{A} t}\right| j\right\rangle=\sum_{n} e^{-\lambda_{n} \gamma t}\left\langle k \mid q_{n}\right\rangle\left\langle q_{n} \mid j\right\rangle .
$$

Since the eigenvalues are positive $\left(\lambda_{n}>0\right.$ for $n>1$ and $\lambda_{1}=0$ ), the long-time limit follows directly: For $t \gg 1$ in the sum of Eq. (7) all exponential terms but one decay quickly to zero. The only term which survives is the one for $\lambda_{1}=0$, with the corresponding eigenstate $\left|q_{1}\right\rangle=\frac{1}{N} \sum_{l}|l\rangle$. Therefore, the long-time limit of all transition probabilities is $\lim _{t \rightarrow \infty} p_{k, j}(t)=1 / N$, which is independent of the connectivity of the network. This means that every CTRW whose transfer matrix follows directly from the connectivity matrix will eventually decay at long times to the equipartition value $1 / N$, a fact which is sometimes referred to as "ground state dominance".

\section{Hamiltonian for CTQW}

Turning now to the quantum-mechanical dynamics, one faces the fact that the transport has to be formulated in Hilbert space. For this one assumes that the states $|j\rangle$ representing the nodes span the whole accessible Hilbert space. As before, it is assumed that the states are orthonormal and complete, i.e., $\langle k \mid j\rangle=\delta_{k, j}, \sum_{j}|j\rangle\langle j|=1$. The dynamics is then governed by a specific Hamiltonian $\boldsymbol{H}$, such that Schrödinger's equation for the transition amplitudes $\alpha_{k, j}(t) \equiv\langle k \mid j ; t\rangle$ reads

$$
\frac{d}{d t} \alpha_{k, j}(t)=-i \sum_{l} H_{k, l} \alpha_{l, j}(t) \text {. }
$$

Similar to the CTRW, the formal solution for the transition amplitudes is given by

$$
\alpha_{k, j}(t)=\left\langle k\left|e^{-i \boldsymbol{H} t}\right| j\right\rangle,
$$

where $e^{-i \boldsymbol{H} t}$ is the quantum-mechanical time-evolution operator. The transition probabilities follow as usual as $\pi_{k, j}(t) \equiv$ $\left|\alpha_{k, j}(t)\right|^{2}$.

Comparing Eq. (8) to Eq. (6) one immediatly notices the very similar structure of the two equations, except for the imaginary unit $i$ appearing in Eq.(8). However, while Eq. (6) is an equation for the transition probabilities $p_{k, j}(t)$, Eq. (8) is an equation for the transition amplitudes $\alpha_{k, j}(t)$.

One can push the similarities further by identifying the quantum-mechanical Hamiltonian $\boldsymbol{H}$ with the classical transfer matrix $\boldsymbol{T}$, i.e., $\boldsymbol{H} \equiv-\boldsymbol{T}$. This approach, pioneered by Farhi and Gutmann in Ref. [21], allows to compare the two extremes of transport on the same topology. On the one hand there is the purely incoherent CTRW, while on the other hand, there is Schrödinger's equation which now defines CTQW. Assuming again that all transition rates between different connected nodes are the same, the Hamiltonian can be directly related to the connectivity, $\boldsymbol{H}=\gamma \boldsymbol{A}$. Therefore, the underlying topological network is determined both for CTRW and for CTQW by the connectivity matrix $\boldsymbol{A}$. This allows to study the role of the connectivity in parallel for CTRW and for CTQW.

Let us donote the eigenvalues of $\boldsymbol{H}$ by $E_{n}$ and the eigenstates of $\boldsymbol{H}$ by $\left|\Psi_{n}\right\rangle$. Evidently, the eigenvalues and eigenstates of $\boldsymbol{H}$ and $\boldsymbol{T}$ are practically the same; nonetheless, we will keep the distinction between $\boldsymbol{H}$ and $\boldsymbol{T}$ due to later purposes. Now, the quantum-mechanical transition probabilities read

$$
\pi_{k, j}(t)=\left|\sum_{n} e^{-i E_{n} t}\left\langle k \mid \Psi_{n}\right\rangle\left\langle\Psi_{n} \mid j\right\rangle\right|^{2}
$$

Unlike the situation for CTRW, for CTQW there is no unique long-time limit of $\pi_{k, j}(t)$ due to the unitary time evolution. In order to compare to the long-time behavior of the CTRW one uses the long time average [70, 71]

$$
\begin{aligned}
& \chi_{k, j} \equiv \lim _{T \rightarrow \infty} \frac{1}{T} \int_{0}^{T} d t \pi_{k, j}(t) \\
& =\sum_{n, m} \delta_{E_{n}, E_{m}}\left\langle k \mid \Psi_{n}\right\rangle\left\langle\Psi_{n} \mid j\right\rangle\left\langle j \mid \Psi_{m}\right\rangle\left\langle\Psi_{m} \mid k\right\rangle,
\end{aligned}
$$

where $\delta_{E_{n}, E_{m}}=1$ if $E_{n}=E_{m}$ and 0 otherwise. The longtime average $\chi_{k, j}$ still depends on the initial and final nodes $j$ and $k$.

\section{Example: A discrete ring}

As an example which illustrates the differences and similarities between CTQW and CTRW we consider a discrete ring of $N$ nodes, whose connectivity matrix is given above in Eq.(3). This one-dimensional structure with the periodic boundary conditions $|N+1\rangle=|1\rangle$ allows for a full analytical solution. When realizing that the matrix $\boldsymbol{A}$ is nothing but a tight-binding matrix for a particle moving in a regular onedimensional crystal, one readily obtains the eigenvalues and eigenstates from a Bloch ansatz [7, 10]. Namely, the Bloch states are linear combinations of the localized states $|j\rangle$ and are given by

$$
\left|\Psi_{\theta}\right\rangle=\frac{1}{\sqrt{N}} \sum_{j=1}^{N} e^{-i \theta j}|j\rangle .
$$

Now the energy is obtained as

$$
E_{\theta}=2-2 \cos \theta
$$

For small $\theta$ the energy is given by $E_{\theta} \approx \theta^{2}$ which resembles the energy spectrum of a free particle. 
By inverting Eq.(12) one may describe the state $|j\rangle$ localized at node $j$ as a Wannier state [7, 10]

$$
|j\rangle=\frac{1}{\sqrt{N}} \sum_{\theta} e^{i \theta j}\left|\Psi_{\theta}\right\rangle .
$$

Since the states $|j\rangle$ span the whole accessible Hilbert space, one has $\langle k \mid j\rangle=\delta_{k j}$ and, via Eq.(12), also $\left\langle\Psi_{\theta^{\prime}} \mid \Phi_{\theta}\right\rangle=\delta_{\theta^{\prime} \theta}$. Then the transition amplitude reads [72]

$$
\begin{aligned}
\alpha_{k j}(t) & =\frac{1}{N} \sum_{\theta, \theta^{\prime}}\left\langle\Psi_{\theta^{\prime}}\left|e^{-i \theta k} e^{-i \boldsymbol{H} t} e^{i \theta^{\prime} j}\right| \Psi_{\theta}\right\rangle \\
& =\frac{1}{N} \sum_{\theta} e^{-i E_{\theta} t} e^{-i \theta(k-j)} .
\end{aligned}
$$

In an analogous way one obtains the classical transition probabilities [72]

$$
p_{k, j}(t)=\frac{1}{N} \sum_{\theta} e^{-\lambda_{\theta} t} e^{-i \theta(k-j)},
$$

because the Bloch states are also the eigenstates $\left|q_{n}\right\rangle$.

The periodic boundary condition for a $1 d$ lattice of size $N$ requires that $\theta=2 n \pi / N$ with $n \in] 0, N]$. Now Eq.(15) is given by

$$
\alpha_{j k}(t)=\frac{e^{-i 2 t}}{N} \sum_{n} e^{i 2 t \cos (2 n \pi / N)} e^{-i 2 \pi n(k-j) / N} .
$$

For small $\theta$, this is directly related to the results obtained for a quantum particle in a box [40-42], because then $E_{n} \sim n^{2}$. Indeed, some features found for the particle in a box can also be found in the case of a CTQW on a discrete ring. For the particle in a box the initial condition is restored after some revival time. In fact the probability to find the particle at a certain position in the box is a periodic function.

Analogously but not completely similarily, for the CTQW on the ring, the initial (localized) condition is only partially restored. Only small rings of sizes $N=1,2,3,4$, and 6 lead to a full revival of the initial condition. All other sizes only lead to partial revivals. The revival time $\tau$ is given by $\alpha_{k, j}(\tau)=\alpha_{k, j}(0)$. Since the transition amplitudes are given as a sum over all modes $n$, see Eq.(17), one has for each mode $n$ its revival time [72]

$$
\tau_{n}=\frac{r \pi}{2}\left[1+\cot ^{2}(n \pi / N)\right],
$$

where $r \in \mathbb{N}$ (without any loss of generality one sets $r=1$ ). From Eq. (18) one finds that $\tau_{n}>\tau_{n+1}$ for $\left.\left.n \in\right] 0, N / 2\right]$ and $\tau_{n}<\tau_{n+1}$ for $\left.\left.n \in\right] N / 2, N\right]$. For certain values of $n$, $\tau_{n}$ will be of order unity, e.g. for $n=N / 2 \tau_{n}=\pi / 2$. However, for $n<<N$, Eq. 18 yields $\tau_{n}=N^{2} / 2 \pi n^{2} \equiv \tau_{0} / n^{2}$, which is analogous to the particle in the box and where $\tau_{0}$ is a universal revival time.

Because the revival times $\tau_{n}$ have large variations in value, one compares these times to the actual time needed by the CTQW for travelling through the lattice. As mentioned earlier, interference effects in the return probability $\pi_{1,1}(t)$ are (a) $\mathrm{N}=21$

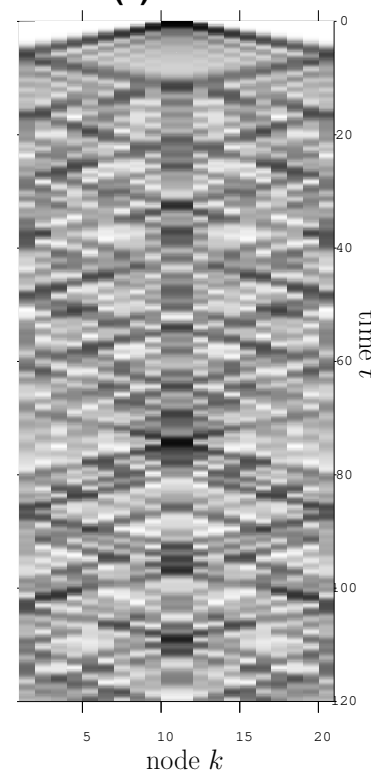

(a) $\mathrm{N}=20$

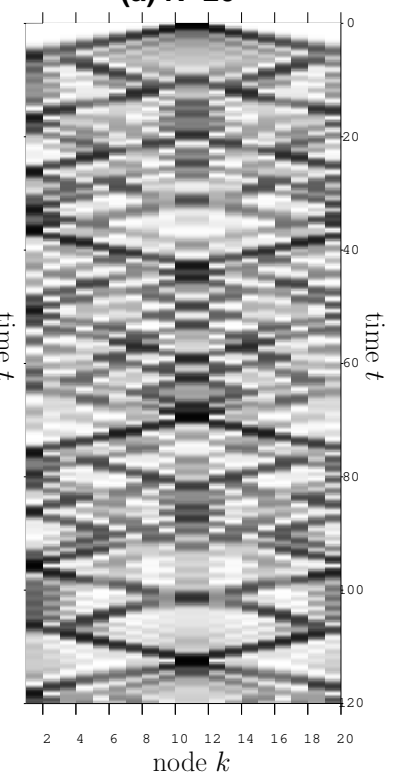

FIG. 1: Contour plot of the probability for a CTQW on a circle of length (a) $N=21$ and (b) $N=20$ over long times $t$. Dark regions denote high probabilities. From [72].

seen after a time $t \approx N / 2$. The first revival time has to be larger than this, because there cannot be any revival unless the wave reaches its starting node again. Our calculations [72] suggest that the first revival time will be of order $\tau_{0}$. From Fig. 1 1 one sees that the first (incomplete) revival occurs for $N=20$ at $t \approx 70>20^{2} / 2 \pi$ and for $N=21$ at $t \approx 75>21^{2} / 2 \pi$.

Since the transition probabilities are easily obtained from the Bloch ansatz, also the long-time averages can be estimated analytically. Depending on the number of nodes in the network, the long-time averages are, [72], for even $N$ :

$$
\chi_{k, j}= \begin{cases}1 / N^{2} & k \neq j \\ 1 / 2 N & k=j \text { and } k=j+N / 2\end{cases}
$$

and for odd $N$ :

$$
\chi_{k, j}= \begin{cases}1 / N^{2} & k \neq j \\ 1 / N & k=j\end{cases}
$$

Thus, unlike in the classical CTRW case the long-time average is not equipartitioned but "remembers" the initial condition. Moreover, also the symmetry arising from the even or odd number $N$ of nodes is reflected in the $\chi_{k, j}$.

\section{EFFICIENCY OF CTQW AND CTRW}

The performance of CTQW and CTRW depends to a large extent on the connectivity of the underlying network, i.e., on its topology. One focuses on the return probabilities $\pi_{j, j}(t)$ and $p_{j, j}(t)$. Now, if these probabilities decay quickly with 
time, the probabilities $1-\pi_{j, j}(t)$ and $1-p_{j, j}(t)$, to be at any but the initial node, increase quickly. This implies a fast transport through the network. In order to make a global statement on the performance, one considers the average return probabilities [73]

$$
\bar{\pi}(t) \equiv \frac{1}{N} \sum_{j} \pi_{j, j}(t) \quad \text { for CTQW }
$$

and

$$
\bar{p}(t) \equiv \frac{1}{N} \sum_{j} p_{j, j}(t) \quad \text { for CTRW. }
$$

Again, a quick decay of $\bar{\pi}(t)[\bar{p}(t)]$ implies a fast propagation through the network, while a slow decay implies a slow propagation.

For CTRW the average return probabilities simplify considerably; one has namely

$$
\begin{aligned}
\bar{p}(t) & =\frac{1}{N} \sum_{n} e^{-\lambda_{n} t}\left\langle\Psi_{n}\left|\sum_{j}\right| j\right\rangle\left\langle j \mid \Psi_{n}\right\rangle \\
& =\frac{1}{N} \sum_{n} e^{-\lambda_{n} t}
\end{aligned}
$$

which only depends on the eigenvalues $\lambda_{n}$ of the transfer matrix $\boldsymbol{T}$ but not on its eigenstates. By making use of the Cauchy-Schwarz inequality one gets a similar expression for CTQW. The average return probabily $\bar{\pi}(t)$ is related to the average return amplitude $\bar{\alpha}(t)$ by [73]

$$
\begin{aligned}
\bar{\pi}(t) & =\frac{1}{N} \sum_{j}\left|\alpha_{j, j}(t)\right|^{2} \\
& \geq\left|\frac{1}{N} \sum_{j} \alpha_{j, j}(t)\right|^{2} \equiv|\bar{\alpha}(t)|^{2} .
\end{aligned}
$$

Similar to $\bar{p}(t)$ for CTRW, $|\bar{\alpha}(t)|^{2}$ only depends on the eigenvalues $E_{n}$ of the Hamiltonian $\boldsymbol{H}$ :

$$
\begin{aligned}
|\bar{\alpha}(t)|^{2} & =\left|\frac{1}{N} \sum_{n} e^{-i E_{n} t}\left\langle\Psi_{n}\left|\sum_{j}\right| j\right\rangle\left\langle j \mid \Psi_{n}\right\rangle\right|^{2} \\
& =\left|\frac{1}{N} \sum_{n} e^{-i E_{n} t}\right|^{2} .
\end{aligned}
$$

Having a quantity which only requires the calculation of the eigenvalues considerably shortens the computation time, especially for very large systems.

Clearly, due to the oscillating terms in Eq.(25), the lower bound $|\bar{\alpha}(t)|^{2}$ will oscillate in most cases. Thus, to compare to the decay of the classical $\bar{p}(t)$ one uses the envelope of $|\bar{\alpha}(t)|^{2}$. Note that when one identifies the Hamiltonian of the CTQW with the transfer matrix of the CTRW, the eigenvalues of both are the same, i.e., $E_{n}=\lambda_{n}$. Thus, formally the difference in dynamics is only due to the different functional form of Eq.(23) and Eq.(25). Nonetheless, this can lead to drastic effects.
As already discussed, for CTRW the long-time limit of the transition probabilies reaches the equipartition value $1 / N$. In the same way, the long-time limit of $\bar{p}(t)$ is given by $1 / N$. In contrast, for CTQW neither $\bar{\pi}(t)$ nor $|\bar{\alpha}(t)|^{2}$ decay to a given value at long times, but rather oscillate around the corresponnding long-time average which for $\bar{\pi}(t)$ is given by [74]

$$
\begin{aligned}
\bar{\chi} & \equiv \lim _{T \rightarrow \infty} \frac{1}{T} \int_{0}^{T} d t \bar{\pi}(t) \\
& =\frac{1}{N} \sum_{n, m} \delta_{\lambda_{n}, \lambda_{m}}\left|\left\langle j \mid \psi_{n}\right\rangle\right|^{2}\left|\left\langle j \mid \psi_{m}\right\rangle\right|^{2} .
\end{aligned}
$$

Again, on can obtain a lower bound which does not depend on the eigenvectors [74]:

$$
\bar{\chi} \geq \frac{1}{N^{2}} \sum_{n, m} \delta_{\lambda_{n}, \lambda_{m}} \equiv \bar{\chi}_{l b} .
$$

\section{A. Examples}

The difference in the global efficiencies of CTQW and CTRW is illustrated by considering two distinct examples: the discrete ring of $N$ nodes and the star with one core node and $N-1$ nodes attached to it, see Fig. 2

The eigenvalues (and also eigenstates) of the discrete ring have already been discussed. From these results it is straightforward to calculate the average return probabilities. Since the ring's eigenstates are Bloch states, one can easily verify that the lower bound $|\bar{\alpha}(t)|^{2}$ is exact, i.e., in this case also $\bar{\pi}(t)$ does not depend on the eigenstates. Therefore, the average return probabilities are given by [73]

$$
\bar{\pi}(t)=\mid \frac{1}{N} \sum_{n=1}^{N} \exp \left[-\left.i 2 t(1-\cos (2 \pi n / N))\right|^{2}\right.
$$

for CTQW and

$$
\bar{p}(t)=\frac{1}{N} \sum_{n=1}^{N} \exp [-2 t(1-\cos (2 \pi n / N))]
$$

for CTRW. In the limit of large $N$, i.e., for continuous $\theta=$ $2 \pi n / N$, one can replace the sums by integrals which leads to

$$
\bar{p}_{\gamma}(t)=\int d E \rho(E) \exp (-E t)
$$

and to

$$
\bar{\pi}_{\gamma}(t)=\left|\int d E \rho(E) \exp (-i E t)\right|^{2},
$$

where $\rho(E)$ is the density of states (DOS). Then one obtains for CTQW $\bar{\pi}(t) \sim\left|J_{0}(2 t)\right|^{2}$, which for $t \gg 1$ can be approximated by $\bar{\pi}(t) \sim \sin ^{2}(2 t+\pi / 4) / t[75]$. Since the maximum of the sine function is 1 , the envelope of $\bar{\pi}(t)$ decays as $t^{-1}$. For CTRW there is only a single sum and no additional quadrature, such that a similar calculation leads to 
$\bar{p}(t) \sim t^{-1 / 2}$. This temporal decay will also be present for finite systems. The time-range over which it will be visible depends on the value of $N$. Obviously, for small $N$ the longtime behavior will be reached faster than for larger $N$.

Thus, we just established that for CTQW on a ring the exponent of the decay power-law is twice as large as the one for CTRW. This fact holds for a wide class of systems. Namely, all networks whose density of states $\rho(E) \equiv \frac{1}{N} \sum_{n=1}^{N} \delta(E-$ $E_{n}$ ) follows a power-law, $\rho(E) \sim\left(E E_{m}-E^{2}\right)^{\nu}$, will show this feature. Here, $E_{m}$ is the maximal eigenvalue (one assumes the minimum eigenvalue to be zero). Since the interest is in the large $t$ behavior, $\bar{p}(t)$ will be mainly determined by the small eigenvalues, such that for $t \gg 1$ one has $\rho(E) \sim E^{\nu}$. It is straighforward to show that for CTRW

$$
\bar{p}(t) \sim t^{-(1+\nu)} .
$$

This scaling feature at not too short times is well known, see, e.g., [76], where $2(1+\nu) \equiv d_{s}$ is sometimes called the spectral or fracton dimension. For CTQW, also $|\bar{\alpha}(t)|^{2}$ will be in general determined by the small eigenvalues. For $\rho(E) \sim E^{\nu}$ one obtains $|\bar{\alpha}(t)|=\bar{p}(t)$. Here, all quantum mechanical oscillations vanish, because one considers only the leading term of the DOS. Therefore, the envelope of the lower bound for CTQW scales as [73]

$$
\operatorname{env}\left[|\bar{\alpha}(t)|^{2}\right] \sim t^{-2(1+\nu)} .
$$

In other cases of interest (e.g., stars, see below) the DOS has highly degenerate eigenvalues [74]. As an extreme case there may exist a single eigenvalue $E_{l}$, whose degeneracy, $D_{l}$, is of the order $\mathcal{O}(N)$, whereas all others are of the order $\mathcal{O}(1)$ or less. By writing

$$
\bar{\alpha}(t)=\frac{1}{N}\left[D_{l} e^{-i E_{l} t}+\sum_{E_{n} \neq E_{l}} D_{n} e^{-i E_{n} t}\right]
$$

one obtains, up to order $\mathcal{O}\left(1 / N^{2}\right)$ [74]:

$$
|\bar{\alpha}(t)|^{2} \approx \frac{D_{l}}{N^{2}}\left\{D_{l}+\sum_{E_{n} \neq E_{l}} D_{n} 2 \cos \left[\left(E_{l}-E_{n}\right) t\right]\right\} .
$$

The first term on the right-hand side of eq. (35) is of order $\mathcal{O}(1)$, while the second term is of order $\mathcal{O}(1 / N)$. Therefore, for few highly degenerate eigenvalues, the lower bound $|\bar{\alpha}(t)|^{2}$ will not show a decay to values which fluctuate about $1 / N$ but will rather fluctuate around $1-1 / N$ at all times. Also, $\bar{\pi}(t)$ will not decay but will fluctuate around the same value, since $|\bar{\alpha}(t)|^{2}$ is a lower bound.

An example of a system with a single highly degenerate eigenvalue is the star, having one core node and $N-1$ nodes directly connected to the core but not to each other, see Fig.2. The eigenvalue spectrum has a very simple structure. There are 3 distinct eigenvalues, namely $E_{1}=0, E_{2}=1$, and $E_{3}=$ $N$, with degeneracies $D_{1}=1, D_{2}=N-2$, and $D_{3}=1$, respectively. Therefore, one gets [74]

$$
\begin{aligned}
\bar{p}(t) & =\frac{1}{N}\left[1+(N-2) \mathrm{e}^{-t}+\mathrm{e}^{-(N-2) t}\right] \\
|\bar{\alpha}(t)|^{2} & =\frac{1}{N^{2}}\left|1+(N-2) \mathrm{e}^{-\mathrm{i} t}+\mathrm{e}^{-\mathrm{i}(N-2) t}\right|^{2} .
\end{aligned}
$$

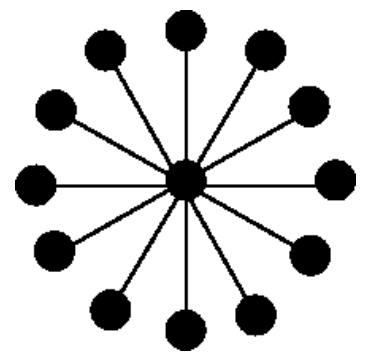

FIG. 2: Star with $N-1=12$ arms.

(a) Ring with $\mathrm{N}=1000$

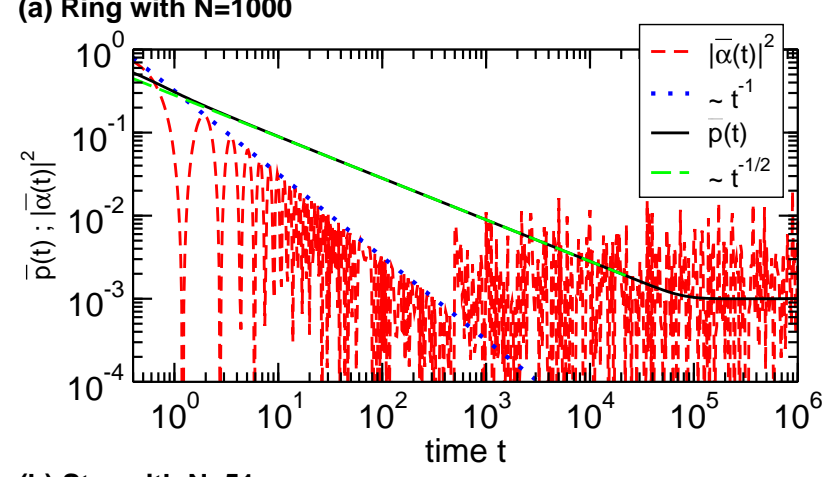

(b) Star with $\mathrm{N}=51$

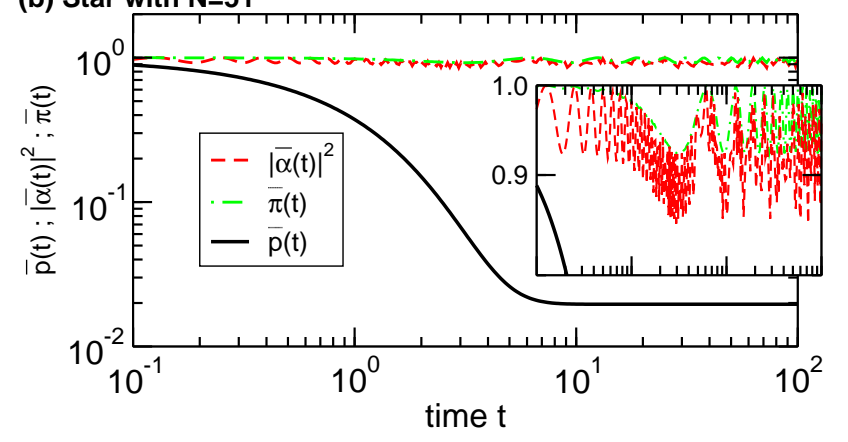

FIG. 3: (Colour on-line). Panel (a): $\bar{p}(t)$ and $|\bar{\alpha}(t)|^{2}$ with the appropriate scaling $t^{-1 / 2}$ and $t^{-1}$, respectively, for a ring of size $N=1000$. Panel (b): $\bar{p}(t), \bar{\pi}(t)$, and $|\bar{\alpha}(t)|^{2}$ for a star with $N=51$ nodes. The inset shows a close-up of $\bar{\pi}(t)$ and $|\bar{\alpha}(t)|^{2}$ in the same time interval. From [74].

Obviously, only the term $|(N-2) \exp (-\mathrm{i} t)|^{2} / N^{2}=(N-$ $2)^{2} / N^{2}$ in Eq. (37) is of order $\mathcal{O}(1)$. All the other terms are of order $\mathcal{O}(1 / N)$ or $\mathcal{O}\left(1 / N^{2}\right)$ and, therefore, cause only small oscillations (fluctuating terms) around or negligible shifts (constant terms) from $(N-2)^{2} / N^{2} \approx 1-1 / N$.

Figure 3 shows the temporal behavior of $\bar{\pi}(t),|\bar{\alpha}(t)|^{2}$, and $\bar{p}(t)$ for a ring (uppper panel) and for a star (lower panel). The difference, especially for CTQW, is dramatic. While in both cases the CTRW decay to the equipartition value $1 / N$, this is not so for the CTQW. For the ring $|\bar{\alpha}(t)|^{2}$ (thus also $\bar{\pi}(t)$ ) follows the $t^{-1}$ decay at intermediate times and oscillates around the long-time average at long times. For the star, on the other hand, there is no decay for both $|\bar{\alpha}(t)|^{2}$ and $\bar{\pi}(t)$. There are only some oscillations around the value $1-1 / N$.

In the two examples above the efficiencies of the two processes are vastly different. For the ring there is a considerable 


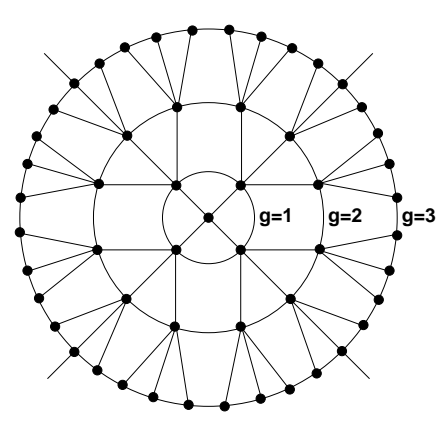

FIG. 4: Spidernet graph of generation $g=3$ with $N=53=1+$ $4 \sum_{k=1}^{g} 3^{k-1}$ nodes.

increase in efficiency for CTQW, because for it the probability to return to the origin decays much faster than for CTRW. The contrary is true for the star. Here, the CTRW still decay while the CTQW remain close to unity. This implies that the probability to visit other places than the initial node is - on average - very low. Note that $\bar{\pi}(t),|\bar{\alpha}(t)|^{2}$, and $\bar{p}(t)$ are averaged over all nodes of the network. For the ring all nodes are equivalent, but the star has the core node which stands out from the rest. Therefore, the temporal evolution is very different when the CTQW starts at the core or at one of the peripheral nodes. When starting at the core, due to the rotational symmetry of the network, the walk can be mapped into a walk involving only two nodes. When starting at the periphery, there is no such simple mapping. Since all but one node are at the periphery, they lead to the poor performance of the CTQW when compared to the CTRW.

Salimi studied the situation over the semi-regular spidernet graph [77]. This graph is build in a hierarchical, radially symmetric manner, i.e., one starts from a single core node and builds up the network generation after generation, as indicated in Fig. 4. The procedure used [73, 74, 77] also leads to powerlaw behaviors for both CTQW and CTRW [77]. When one starts at the core node, one can map the dynamics onto a line, where all the states corresponding to the nodes belonging to the same generation are summed up to form a new state, representing this generation. Salimi showed that in this case the return to the origin (core node) follows a power-law which for CTRW goes as $t^{-3 / 2}$ while for CTQW it goes as $t^{-3}$. Therefore, a behavior similar to that discussed around Eqs. (32) and (33) is also found here, since the exponent of the CTQW decay is twice as large as the exponent of the CTRW decay.

\section{CTQW ON NETWORKS}

\section{A. Deterministic networks}

\section{Two dimensional regular networks}

CTQW on regular $2 d$ structures carry over many of the properties of their $1 d$ counterparts, since $2 d$ regular networks ca be envisaged to be the direct product of two $2 d$ structures, vide infra. However, some care is in order, since the symme-

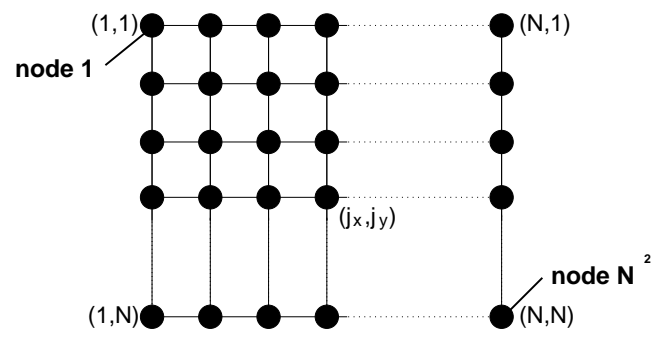

FIG. 5: Sketch of a square network arranged as a regular lattice with the appropriate numbering of the nodes. Note that the actual geometrical realization can be much more flexible, see text for details. From [71].

tries observed in 1d are not always observed in 2d [71].

Consider now $2 d$ regular structures of side length $N$, thus, they contain $N^{2}$ nodes giving rise to $N^{2}$ basis states [71]. In a pair notation one sets $|\boldsymbol{j}\rangle=\left|j_{x}, j_{y}\right\rangle$, where $j_{x}$ and $j_{y}$ are integer labels in the two directions, with $j_{x}, j_{y} \in[1, N]$, see Fig.5. This labeling of the states is not to be confused with the labeling of the adjacency matrix. Note that capital bold letters denote matrices, while small bold letters denote the nodes and the states.

The focus in solid state physics is on systems where Born - von Karman periodic boundary conditions (PBC) are assumed. Now, for an internal site of the network (not on an edge or in a corner), the Hamiltonian acting on a state $|\boldsymbol{j}\rangle=\left|j_{x}, j_{y}\right\rangle$ reads

$$
\begin{aligned}
\boldsymbol{H}\left|j_{x}, j_{y}\right\rangle & =2\left|j_{x}, j_{y}\right\rangle-\left|j_{x}+1, j_{y}\right\rangle-\left|j_{x}-1, j_{y}\right\rangle \\
& +2\left|j_{x}, j_{y}\right\rangle-\left|j_{x}, j_{y}+1\right\rangle-\left|j_{x}, j_{y}-1\right\rangle
\end{aligned}
$$

PBC extend this equation to all the sites of the network by interpreting every integer $j_{x}$ and $j_{y}$ to be taken modulus $N$. With this generalization, the time independent SE

$$
\boldsymbol{H}\left|\Psi_{\boldsymbol{\theta}}\right\rangle=E_{\boldsymbol{\theta}}\left|\Psi_{\boldsymbol{\theta}}\right\rangle
$$

admits (similar to the 1d case) the following Bloch eigenstates

$$
\left|\Psi_{\boldsymbol{\theta}}\right\rangle=\frac{1}{N} \sum_{j_{x}, j_{y}=1}^{N} e^{-i(\boldsymbol{\theta} \cdot \boldsymbol{j})}|\boldsymbol{j}\rangle .
$$

as solutions, where $\boldsymbol{\theta} \cdot \boldsymbol{j}$ stands for the scalar product with $\boldsymbol{\theta}=\left(\theta_{x}, \theta_{y}\right)$. The usual Bloch relation can be obtained by projecting $\left|\Psi_{\boldsymbol{\theta}}\right\rangle$ on the state $|\boldsymbol{j}\rangle$ such that $\Psi_{\boldsymbol{\theta}}(\boldsymbol{j}) \equiv\left\langle\boldsymbol{j} \mid \Psi_{\boldsymbol{\theta}}\right\rangle=$ $e^{-i(\boldsymbol{\theta} \cdot \boldsymbol{j})} / N$, thus $\Psi_{\boldsymbol{\theta}}\left(j_{x}+1, j_{y}+1\right)=e^{-i\left(\theta_{x}+\theta_{y}\right)} \Psi_{\boldsymbol{\theta}}\left(j_{x}, j_{y}\right)$. The PBC restrict the allowed values of $\theta$. In the present case [71] (side length $N)$, the PBC require that $\Psi_{\boldsymbol{\theta}}\left(N+1, j_{y}\right)=$ $\Psi_{\boldsymbol{\theta}}\left(1, j_{y}\right)$ and $\Psi_{\boldsymbol{\theta}}\left(j_{x}, N+1\right)=\Psi_{\boldsymbol{\theta}}\left(j_{x}, 1\right)$. It follows that one must have $\theta_{x}=2 n \pi / N$ and $\theta_{y}=2 l \pi / N$, where $n$ and $l$ are integers and $n, l \in[1, N]$. It is now a simple matter to verify that the $\left|\Psi_{\boldsymbol{\theta}}\right\rangle$ also obey $\left\langle\Psi_{\boldsymbol{\theta}} \mid \Psi_{\boldsymbol{\theta}^{\prime}}\right\rangle=\delta_{\boldsymbol{\theta}, \boldsymbol{\theta}^{\prime}}$ and $\sum_{\theta}\left|\Psi_{\theta}\right\rangle\left\langle\Psi_{\theta}\right|=1$.

Furthermore, from Eqs. (39) and (40) the energy is obtained as

$$
E_{\boldsymbol{\theta}}=4-2 \cos \theta_{x}-2 \cos \theta_{y}=E_{\theta_{x}}+E_{\theta_{y}},
$$


with $E_{\theta_{x}}=2-2 \cos \theta_{x}$ and $E_{\theta_{y}}=2-2 \cos \theta_{y}$. Under PBC the two-dimensional eigenvalue problem separates into two one-dimensional problems.

The transition amplitude at time $t$ from state $|\boldsymbol{j}\rangle$ to state $|\boldsymbol{k}\rangle$ is now, using Eq. (40) twice, [71]:

$$
\begin{aligned}
\alpha_{\boldsymbol{k}, \boldsymbol{j}}(t) & =\frac{1}{N^{2}} \sum_{\boldsymbol{\theta}, \boldsymbol{\theta}^{\prime}}\left\langle\Psi_{\boldsymbol{\theta}^{\prime}}\left|e^{-i\left(\boldsymbol{\theta}^{\prime} \cdot \boldsymbol{k}\right)} e^{-i \boldsymbol{H} t} e^{i(\boldsymbol{\theta} \cdot \boldsymbol{j})}\right| \Psi_{\boldsymbol{\theta}}\right\rangle \\
& =\frac{1}{N^{2}} \sum_{\boldsymbol{\theta}} e^{-i E_{\boldsymbol{\theta}} t} e^{-i \boldsymbol{\theta} \cdot(\boldsymbol{k}-\boldsymbol{j})}
\end{aligned}
$$

In the limit $N \rightarrow \infty$, the sums in Eq. (42) may be changed to integrals; by making use of Eq.41) one obtains

$$
\begin{aligned}
\lim _{N \rightarrow \infty} \alpha_{\boldsymbol{k}, \boldsymbol{j}}(t)= & \frac{e^{-i 4 t}}{4 \pi^{2}} \int_{-\pi}^{\pi} d \theta_{x} e^{-i \theta_{x}\left(k_{x}-j_{x}\right)} e^{i 2 t \cos \theta_{x}} \\
& \times \int_{-\pi}^{\pi} d \theta_{y} e^{-i \theta_{y}\left(k_{y}-j_{y}\right)} e^{i 2 t \cos \theta_{y}},
\end{aligned}
$$

such that

$$
\lim _{N \rightarrow \infty} \alpha_{\boldsymbol{k}, \boldsymbol{j}}(t)=i^{k_{x}-j_{x}} i^{k_{y}-j_{y}} e^{-i 4 t} J_{k_{x}-j_{x}}(2 t) J_{k_{y}-j_{y}}(2 t)
$$

where $J_{n}(x)$ is the Bessel function of the first kind [78]. Thus, on a network topologically equivalent to a square lattice with PBC the transition amplitude between the nodes $j$ and $\boldsymbol{k}$ is given by

$$
\lim _{N \rightarrow \infty} \pi_{\boldsymbol{k}, \boldsymbol{j}}(t)=\left[J_{k_{x}-j_{x}}(2 t) J_{k_{y}-j_{y}}(2 t)\right]^{2} .
$$

Now, for finite networks the long-time average $\chi_{k, j}$ gives more insight into the dynamics for different network sizes $N$. In the $2 \mathrm{~d}$ case one has [71]

$$
\begin{aligned}
\chi_{\boldsymbol{k}, \boldsymbol{j}} & =\lim _{T \rightarrow \infty} \frac{1}{T} \int_{0}^{T} d t\left|\sum_{n}\left\langle\boldsymbol{k}\left|e^{-i \boldsymbol{H} t}\right| \boldsymbol{q}_{n}\right\rangle\left\langle\boldsymbol{q}_{n} \mid \boldsymbol{j}\right\rangle\right|^{2} \\
& =\sum_{n, m}\left\langle\boldsymbol{k} \mid \boldsymbol{q}_{n}\right\rangle\left\langle\boldsymbol{q}_{n} \mid \boldsymbol{j}\right\rangle\left\langle\boldsymbol{j} \mid \boldsymbol{q}_{m}\right\rangle\left\langle\boldsymbol{q}_{m} \mid \boldsymbol{k}\right\rangle \\
& \times\left(\lim _{T \rightarrow \infty} \frac{1}{T} \int_{0}^{T} d t e^{-i\left(\lambda_{n}-\lambda_{m}\right) \gamma t}\right) \\
& =\sum_{n, m} \delta_{\lambda_{n}, \lambda_{m}}\left\langle\boldsymbol{k} \mid \boldsymbol{q}_{n}\right\rangle\left\langle\boldsymbol{q}_{n} \mid \boldsymbol{j}\right\rangle\left\langle\boldsymbol{j} \mid \boldsymbol{q}_{m}\right\rangle\left\langle\boldsymbol{q}_{m} \mid \boldsymbol{k}\right\rangle .
\end{aligned}
$$

One notes that the integral in Eq.46a equals 1 if $\lambda_{n}=\lambda_{m}$ and 0 otherwise, i.e., it equals $\delta_{\lambda_{n}, \lambda_{m}}$. Given that some eigenvalues of $\boldsymbol{H}$ are degenerate, the sum in Eq.46b) can contain terms belonging to different eigenstates $\left|\boldsymbol{q}_{n}\right\rangle$ and $\left|\boldsymbol{q}_{m}\right\rangle$. Equation 46b provides a numerically very efficient way of computing the $\chi_{\boldsymbol{k}, \boldsymbol{j}}$. Remarkably, one finds that the $\chi_{\boldsymbol{k}, \boldsymbol{j}}$ depend in an unexpected way on the exact value of the size $N$ of the finite network under study.
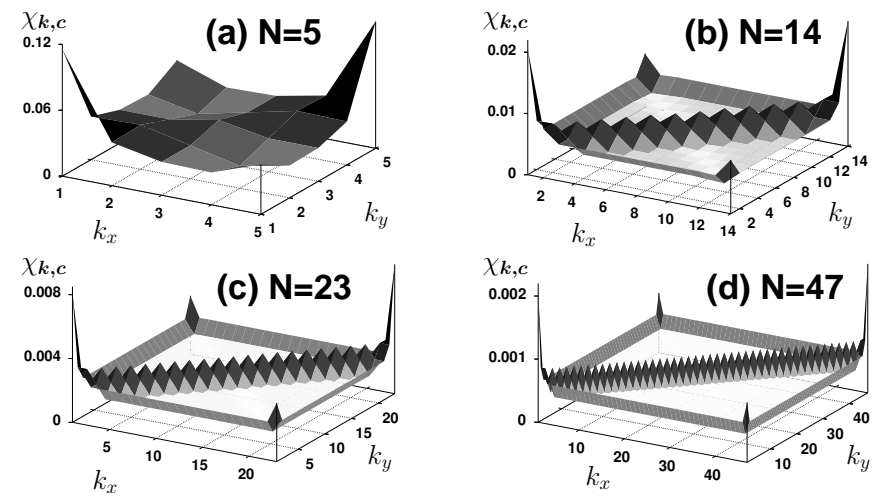

FIG. 6: LPs $\chi_{k, c}$ to be at node $\boldsymbol{k}$ when starting at the corner node $\boldsymbol{c}=(1,1)$ for networks of sizes (a) $N=5$, (b) $N=14$, (c) $N=23$, and (d) $N=47$. From [71].
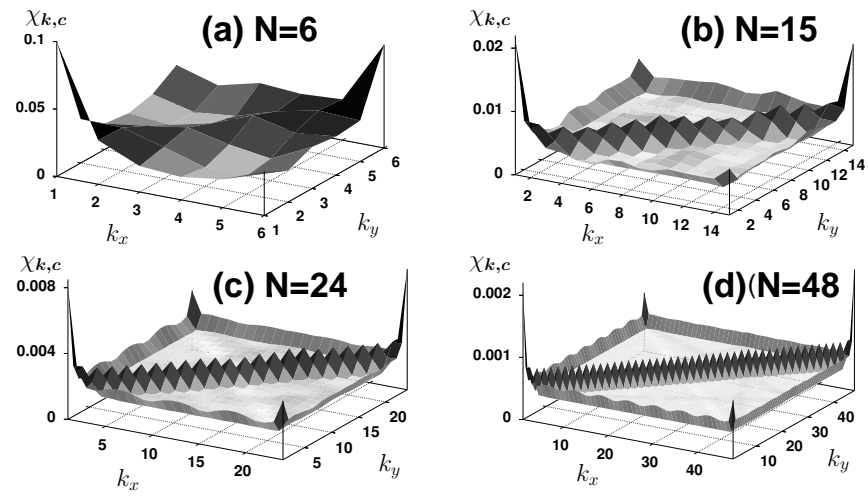

FIG. 7: LPs $\chi_{\boldsymbol{k}, \boldsymbol{c}}$ to be at node $\boldsymbol{k}$ when starting at the corner node $\boldsymbol{c}=(1,1)$ for networks of sizes (a) $N=6$, (b) $N=15$, (c) $N=24$, and (d) $N=48$. One may note the asymmetries by comparing to Fig[6 From [71].

When starting at a corner node $c=(1,1)$, one often finds that the LPs for the starting node and its "mirror" node $\boldsymbol{o c}=(N, N)$ are equal. Figure 6 shows the $\chi_{\boldsymbol{k}, \boldsymbol{c}}$ obtained by going from the corner node $\boldsymbol{c}=(1,1)$ to the other nodes for networks of sizes $N=5, N=14, N=23$, and $N=47$.

However, for some particular network sizes the distributions of the LPs turn out to be asymmetric. For instance, for a network of size $N=15$ the LP $\chi_{o c, c}$ for the CTQW starting at node $c$ to be at the opposite corner node $\boldsymbol{o c}$ is less than the LP $\chi_{c, c}$ to be at the initial node. The same is true for the nodes along the edges of the network. Figure 7 shows that such asymmetries occur for networks of sizes $N=6, N=15$, $N=24$, and $N=48$ (the asymmetries are best seen by looking at $\chi_{c, c}$ and $\left.\chi_{o c, c}\right)$. The smallest network where asymmetries in the distribution of the LPs are detected has $N=6$. The next ones are found for $N=12,15,18,21,24,30,36, \cdots$.

An asymmetric LP distribution is particularly evident in the difference between $\chi_{\boldsymbol{c}, \boldsymbol{c}}$ and $\chi_{\boldsymbol{c}, \boldsymbol{c}, \boldsymbol{c}}$. Thus, as an overview Fig 8 presents as a function of $N$ a plot of the $\left(\chi_{c, c}-\right.$ $\left.\chi_{o c, c}\right) N^{2}$ values obtained. Note that all $N$ values in Fig 8 for which $\left(\chi_{c, c}-\chi_{o c, c}\right) \neq 0$ are divisible by 3 . However, the converse is not true, one finds symmetric LP distributions for 


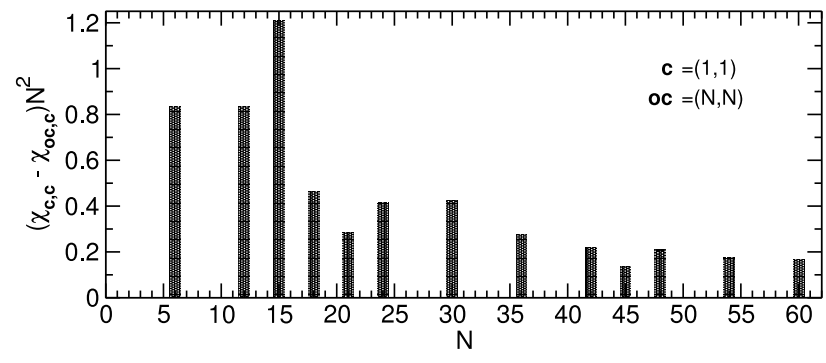

FIG. 8: Differences between the LPs for CTQW that start at $c=$ $(1,1)$ to be at $\boldsymbol{c}, \chi_{\boldsymbol{c}, \boldsymbol{c}}$, or to be at its "mirror" node $\boldsymbol{o c}=(N, N)$, $\chi_{o c, c}$, as a function of the network size $N$, for $1 \leq N \leq 60$. From [71].

the networks with $N=3,9,27,33,39, \cdots$.

The above effect is also observed for non-square networks, where the number of nodes in the two directions are not equal [79]. While for periodic boundary conditions in both directions there is no asymmetry, there are observable asymmetries both for periodic and for open boundary conditions in only one direction as well as for open boundary conditions in both directions.

One now fixes $N$ to be $N=15$ and varies $M$, taking $4 \leq M \leq 30$. Figure 9 a) shows for rectangles the difference between the LPs on the initial corner $\chi_{c, c}$ and on the corner $\chi_{o c, c}$. In the analysed range, $4 \leq M \leq 30$, the value $\chi_{c, c}-\chi_{o c, c}$ displays varying patterns. For $4 \leq M \leq 13$ one can associate $\chi_{c, c}-\chi_{o c, c}=0$ to odd values of $M$ and $\chi_{c, c}-\chi_{o c, c} \neq 0$ to even values of $M$; for larger $M$ the situation becomes more complex. Figure 9 (b) displays the situation for cylinders with $N=15$ and $4 \leq M \leq 30$. Here the plot shows $\chi_{\boldsymbol{c}, \boldsymbol{c}}-\chi_{\boldsymbol{c}_{y}, \boldsymbol{c}}$, with $c_{y} \equiv(1, N)$. Figure 9 (c) shows the situation for cylinders with $M=15$ and $4 \leq N \leq 30$. This last case looks quite regular, with non-vanishing values only for $N=10,15$, and 30 . Hence, Figs. 9 (b) and 9 (c) $(N<M)$ show for cylinders that changes in radius lead to more asymmetric situations than changes in length.

An indication of the origin of the asymmetries is obtained by briefly reviewing some of the details of the calculations of the LPs. Since the Hamiltonian of the problem separates in the two directions, it is easy to show that the transition probabilities $\pi_{\boldsymbol{k}, \boldsymbol{j}}(t)$ can be written as the product of the two separate probabilities for each direction [79], i.e.

$$
\begin{aligned}
\pi_{\boldsymbol{k}, \boldsymbol{j}}(t) & =\pi_{k_{x}, j_{x}}(t) \pi_{k_{y}, j_{y}}(t) \\
& =\left|\alpha_{k_{x}, j_{x}}(t)\right|^{2}\left|\alpha_{k_{y}, j_{y}}(t)\right|^{2},
\end{aligned}
$$

with $\alpha_{k_{x}, j_{x}}(t)=\sum_{\theta_{x}} \exp \left(-i \lambda_{\theta_{x}} t\right)\left\langle k_{x} \mid \Psi_{\theta_{x}}\right\rangle\left\langle\Psi_{\theta_{x}} \mid j_{x}\right\rangle$, and similarly for the $y$-direction.

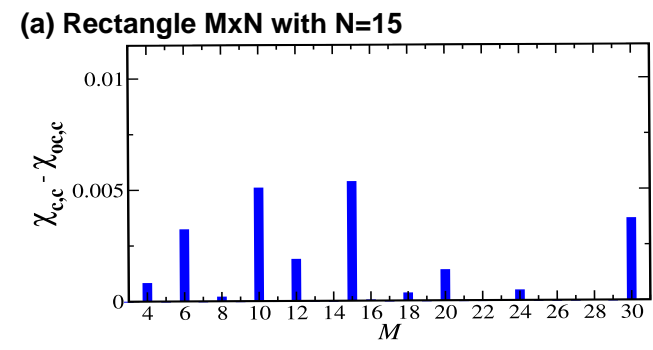

(b) Cylinder $\mathrm{MxN}$ with $\mathrm{N}=15$

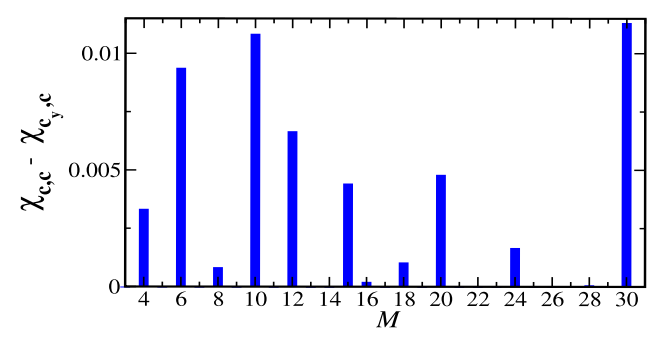

(c) Cylinder MxN with $M=15$

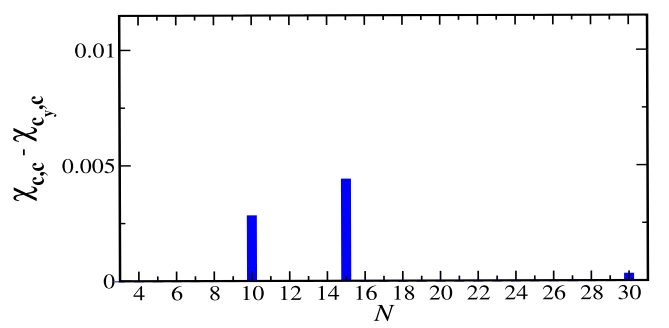

FIG. 9: (a) Rectangles $M \times N$ with $N=15$ and varying $M$ : Differences between the LPs for CTQW that start at $\boldsymbol{c}=(1,1)$ to be at $\boldsymbol{c}$, $\chi_{\boldsymbol{c}, \boldsymbol{c}}$, and to be at $\boldsymbol{o c}=(M, N), \chi_{\boldsymbol{o c}, \boldsymbol{c}}$. (b) Cylinders $M \times N$ with $N=15$ and varying $M$ : Differences between the LPs for CTQW that start at $\boldsymbol{c}=(1,1)$ to be at $\boldsymbol{c}$, and to be at the node $\boldsymbol{c}_{\boldsymbol{y}}=(1, N)$. (c) Cylinders $M \times N$ with $M=15$ and varying $N$ : Differences between the LPs for CTQW that start at $\boldsymbol{c}=(1,1)$ to be at $\boldsymbol{c}$, and to be at the node $\boldsymbol{c}_{\boldsymbol{y}}=(1, N)$. From [79].

Now, according to Eq. (46a), the LPs are given by [79]

$$
\begin{aligned}
\chi_{\boldsymbol{k}, \boldsymbol{j}}= & \lim _{T \rightarrow \infty} \frac{1}{T} \int_{0}^{T} d t \pi_{k_{x}, j_{x}}(t) \pi_{k_{y}, j_{y}}(t) \\
= & \sum_{\theta_{x}, \theta_{x}^{\prime}, \theta_{y}, \theta_{y}^{\prime}} F_{\boldsymbol{k}, \boldsymbol{j}} \lim _{T \rightarrow \infty} \frac{1}{T} \int_{0}^{T} d t \\
& \times \exp \left[-i t\left(\lambda_{\theta_{x}}-\lambda_{\theta_{x}^{\prime}}+\lambda_{\theta_{y}}-\lambda_{\theta_{y}^{\prime}}\right)\right],
\end{aligned}
$$

where $F_{k, j}$ is a time independent function, which depends on the eigenstates associated with $\theta_{x}, \theta_{x}^{\prime}, \theta_{y}$, and $\theta_{y}^{\prime}$. Because of the limit in the time integral in Eq. (48), there are only contributions to $\chi_{\boldsymbol{k}, \boldsymbol{j}}$ if a value $\left(\lambda_{\theta_{x}}-\lambda_{\theta_{x}^{\prime}}\right)$ for the $x$-direction has a counterpart $-\left(\lambda_{\theta_{y}}-\lambda_{\theta_{y}^{\prime}}\right)$ in the $y$-direction.

A careful analysis of the differences $\left(\lambda_{\theta_{x}}-\lambda_{\theta_{x}^{\prime}}\right)$ indicates where the asymmetries stem from. For finite chains one ob- 
tains $\left(\lambda_{\theta_{x}}-\lambda_{\theta_{x}^{\prime}}\right)=2 \cos \theta_{x}^{\prime}-2 \cos \theta_{x}$. For simplicity one considers now finite $N \times N$ networks with OBCs, see [71], because then the eigenvalues are the same in both directions. It turns out that for $\theta_{x} \neq \theta_{x}^{\prime}$ the value $\left(\lambda_{\theta_{x}}-\lambda_{\theta_{x}^{\prime}}\right)$ appears only once or twice for all symmetric cases. However, for the asymmetric cases, some of the $\left(\lambda_{\theta_{x}}-\lambda_{\theta_{x}^{\prime}}\right)$ values (again for $\theta_{x} \neq \theta_{x}^{\prime}$ ) appear more than twice. Therefore, there are more contributions to $\chi_{\boldsymbol{k}, \boldsymbol{j}}$ in the asymmetric cases than in the symmetric cases.

\section{Star-like networks}

Networks which are not regular but, yet, have symmetry properties which can be exploited, are star-like networks. As already mentioned in Sec. IIIA and also discussed in [73, 74], these networks consist of a central core node (with label 1) to which each of the remaining $N-1$ nodes is attached by an individual bond, see Fig. 22 The Hamiltonian has then the following structure

$$
\boldsymbol{H}=(N-1)|1\rangle\langle 1|+\sum_{j=2}^{N}(|j\rangle\langle j|-| 1\rangle\langle j|-| j\rangle\langle 1|) \text {. }
$$

Using the Gram-Schmidt orthonormalization procedure, $\mathrm{Xu}$ obtained the eigenstates of $\boldsymbol{H}$ [80]

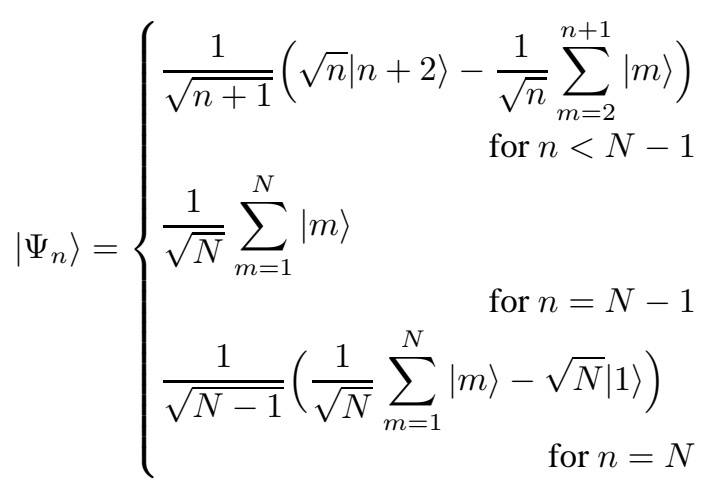

This yields analytic expressions for the transition probabilities, e.g.,

$$
\pi_{1,1}(t)=\frac{N^{2}-2 N+2}{N^{2}}+\frac{2(N-1)}{N^{2}} \cos (N t) .
$$

One easily verifies that for $\cos (N t)=1$, i.e., $t=2 \pi r / N$ (where $r$ is an integer), there is a perfect revival. Moreover, for $t=(2 r+1) \pi / N$ all the probabilitiy is distributed over all but the core node. This is not true if one starts the CTQW at any of the other $(N-1)$ nodes, then one has

$$
\begin{aligned}
\pi_{2,2}(t) & =\left[\left(N^{4}-4 N^{3}+5 N^{2}-2 N+2\right)\right. \\
& +\left(2 N^{3}-6 N^{2}+4 N\right) \cos (t) \\
& +\left(2 N^{2}-4 N\right) \cos ((N-1) t) \\
& +(2 N-2) \cos (N t)] \frac{1}{N^{2}(N-1)^{2}}
\end{aligned}
$$

(a) 6-fold finite path star graph
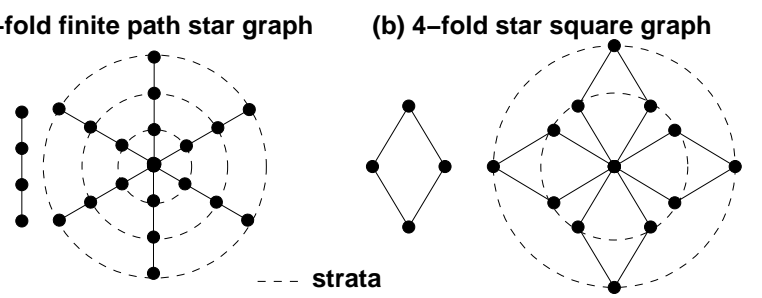

FIG. 10: (a) Star with 6 arms composed of finite segments of length 4. (b) Star with 4 arms composed of circles of length 4 . The dashed lines are guides to the eye, connecting all nodes belonging to given strata, see text.

where, without loss of generality, the node 2 was chosen as initial node. One sees, especially for large $N$, that the probabilities are mainly localized on the initial node. For all initial conditions, the classical transition probabilities $p_{k, j}(t)$ approach the equipartition value $1 / N$.

Having determined the probabilities to return or to still be at the origin allows to calculate the average return probability $\bar{\pi}(t)$, see Sec. III] One has thus

$$
\bar{\pi}(t)=\frac{1}{N} \sum_{k=1}^{N} \pi_{k, k}(t)=\frac{1}{N}\left[\pi_{1,1}(t)+(N-1) \pi_{2,2}(t)\right],
$$

which leads to the results given in Eq. (37).

The strong dependence on the initial conditions also carries over to the long-time averages $\chi_{k, j}$. One obtains namely

$$
\begin{aligned}
& \chi_{1,1}=\left(N^{2}-2 N+2\right) / N^{2}, \\
& \chi_{2,1}=2 / N^{2} \\
& \chi_{2,2}=\left(N^{4}-4 N^{3}+5 N^{2}-2 N+2\right) /\left[N^{2}(N-1)^{2}\right], \\
& \chi_{3,2}=2\left(N^{2}-N+1\right) /\left[N^{2}(N-1)^{2}\right] .
\end{aligned}
$$

Thus, for large $N$ the probability will be concentrated at the initial node.

In a slightly more general setup, Salimi considered CTQW on networks (called star graphs in [81]) with are built from several sub-networks such that all sub-networks share a single node, see Fig. 10. The adjacency matrix is then a direct product of the separate adjacency matrices with additonal entries for the newly created bonds.

By considering so-called strata, i.e., sets formed by all the nodes at the same chemical distance from the central node, see the dashed lines in Fig.10, Salimi calculated the transition probabilities to go from the central node to the different strata. For a star with $N$ arms each of which having 2 nodes, see 
Fig. 2 in [81], he obtains for the transition probabilities

$$
\begin{aligned}
\pi_{1,1}(t) & =\left|\frac{1+N \cos (\sqrt{N+1} t)}{N+1}\right|^{2}, \\
\pi_{2,1}(t) & =\left|\frac{\sqrt{N} \sin (\sqrt{N+1} t)}{\sqrt{N+1}}\right|^{2}, \\
\text { and } \pi_{3,1}(t) & =\left|\frac{N(1-\cos (\sqrt{N+1} t)}{(N+1) \sqrt{N}}\right|^{2} .
\end{aligned}
$$

In the case of a star made out of rings of length 4 , such that all rings share a single node, there are again 2 strata and the central node, see Fig. 10 b) and [81]. The transition probabilities are given by

$$
\begin{aligned}
\pi_{1,1}(t) & =\left|\frac{1+N \cos (\sqrt{2(N+1)} t)}{N+1}\right|^{2}, \\
\pi_{2,1}(t) & =\left|\frac{\sqrt{N} \sin (\sqrt{2(N+1)} t)}{\sqrt{N+1}}\right|^{2}, \\
\text { and } \pi_{3,1}(t) & =\left|\frac{N(1-2 \cos (\sqrt{N+1} t)}{2(N+1) \sqrt{N}}\right|^{2},
\end{aligned}
$$

results very similar to the above.

For strata more distant from the core, one can still compute the transition probabilities for different $N$. Evidently, the $N=1$ case corresponds to a semi-infinite line, while the $N=2$ case is equivalent to the infinite line, i.e., the transition probabilities are given by Bessel functions.

Letting $N$ go to infinity, Salimi showed that in all cases considered above the transition probabilities reduce to

$$
\begin{aligned}
\pi_{1,1}(t) & =\cos ^{2} t, \\
\pi_{2,1}(t) & =\sin ^{2} t, \\
\text { and } \pi_{3,1}(t) & =0,
\end{aligned}
$$

which is equivalent to the result for a dimer, i.e., a complete graph consisting of two nodes.

\section{Complete graph}

The complete graph (for finite $N$ ), where all nodes are mutually connected with each other, shares some properties with the star graph. The Hamiltonian now reads

$$
\boldsymbol{H}=(N-1) \sum_{j=1}^{N}|j\rangle\left\langle j\left|-\sum_{k \neq j}\right| j\right\rangle\langle k| .
$$

The graph has two distinct eigenvalues $E_{N}=0$ and $E_{n}=N$ for $n=1, \ldots, N-1$. Xu showed that, similar to the star [73, 74, 80], one can calculate the eigenstates using the GramSchmidt orthonormalization procedure [80]. For the transition probabilities he then obtains

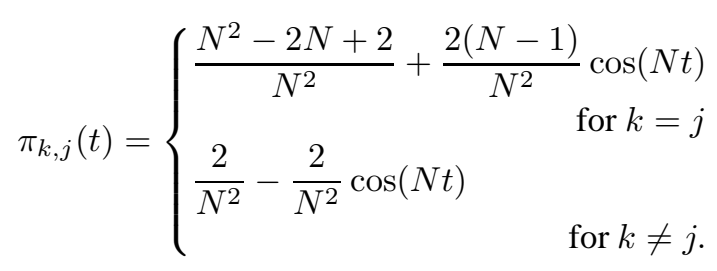

Therefore, the transition probabilities have exactly the same form as the ones for the star graph, when the excitation starts at the core.

\section{Dendrimers}

Star graphs of length 1 can also be viewed as being dendrimers (Cayley-trees) of first generation. The stucture of dendrimers is exemplified in Fig. 11 for dendrimers of generations $G=2$ and $G=3$, with functionality $f=3$, see also [82]. In general, the functionality $f$ gives the number of bonds emanating from each node; the generation $G$ refers to all the nodes whose shortest distance (in bond units) from the central node is not larger than $G$. Note that the number of nodes belonging to the $g$-th generation (where $G \geq g \geq 1$ ) is $3 \cdot 2^{g-1}$ and that it grows exponentially with $g$. Moreover, the total number of nodes in the dendrimer of generation $G$ is $N=3 \cdot 2^{G}-2$.

The connectivity matrix of these dendrimers has a very simple structure. One has $A_{i i}=3$ for all the nodes in the first $G-1$ generations and $A_{i i}=1$ for the nodes in generation $G$. The bonds are represented by the off-diagonal matrix elements $A_{i j}$. Here, every node in generation $g \geq 1$ is connected to two consecutively numbered nodes in generation $g+1$ and to one node in generation $g-1$.

The eigenmodes of such dendrimers were studied in [83]. There, for the dendrimers of generations $G=1$ and $G=2$, the eigenvalues and eigenvectors of $\boldsymbol{A}$ were explicitly calculated. The eigenvectors determine the eigenmodes of the dendrimer, see e.g. Fig. 3 of [83]. It was further shown that there are $G+1$ nondegenerate eigenvalues, one of which is always $\lambda_{0}=0$. (a) $G=2$

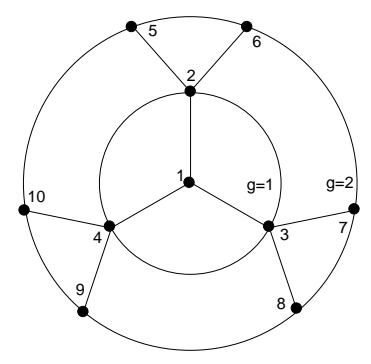

(b) $\mathrm{G}=3$

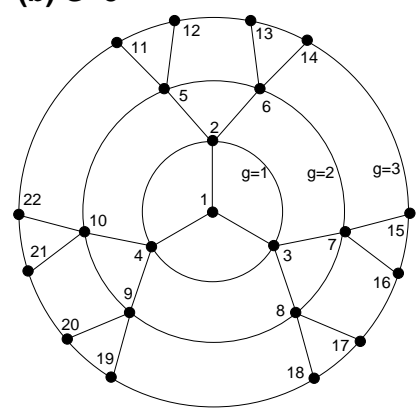

FIG. 11: Dendrimers of functionality $f=3$ and generation $G=2$ (left) and $G=3$ (right). From [82]. 
(a)

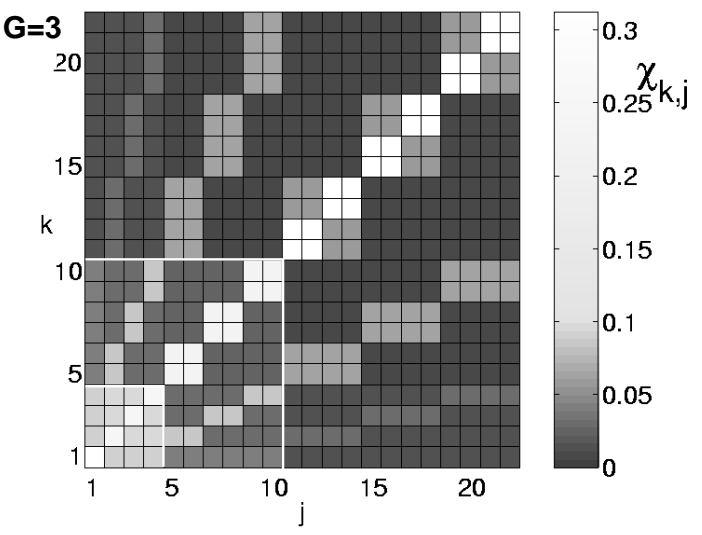

(b)

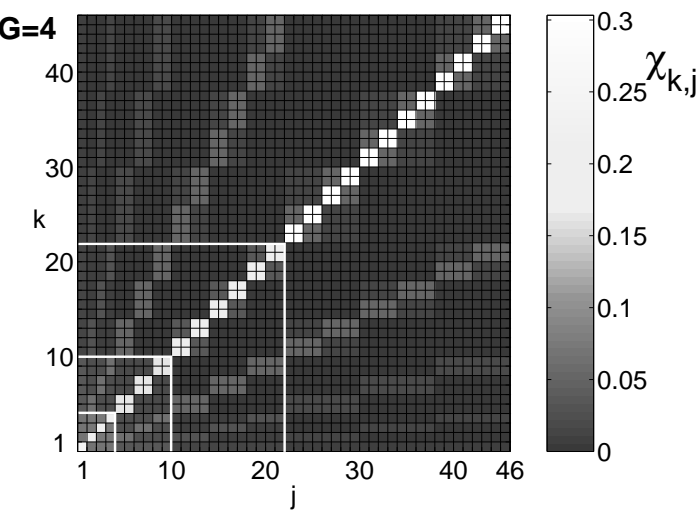

FIG. 12: Limiting probabilities for dendrimers with (a) $G=3$ and (b) $G=4$. The white lines indicate the limiting distributions for the dendrimers of generations smaller than $G$. From [82].

When an excitation starts at the central node 1 , the dynamics of this excitation over the dendrimer can be mapped onto a line. Remarkably, for the $G=2$ dendrimer the transition probabilities are fully periodic when the coherent excitation starts from the central node 1 (the same holds for the $G=1$ dendrimer, too). Note that due to rotational symmetry, the transition probabilities from the central node to nodes belonging to the same generation are equal. Because of this one only has to list three different transition probabilities. It follows that there is a perfect revival of the initial state, which resembles results obained for continuous [40,41] and discrete quantum carpets [72, 84].

If the initial excitation is placed at one of the nodes of the outermost generation $g=G$ of the dendrimer, the picture changes. Classically, the propagation through the dendrimer gets to be much slower than in the previous case, see e.g. $[58,85,86]$. Nevertheless, eventually the excitation will classically propagate through the whole graph and in the long time limit the probability will be equipartitioned among all nodes. Quantum mechanically this effect is even more dramatic. The main fraction of $\pi_{k, j}(t)$ stays in a small region closely connected by bonds to the initial node $j$, and the transfer to other sites is highly unlikely. Also at long times the limiting probability for the excitation to reach the other branches of the dendrimer stays very low.

Classically, the LP is equipartitioned among all the nodes,

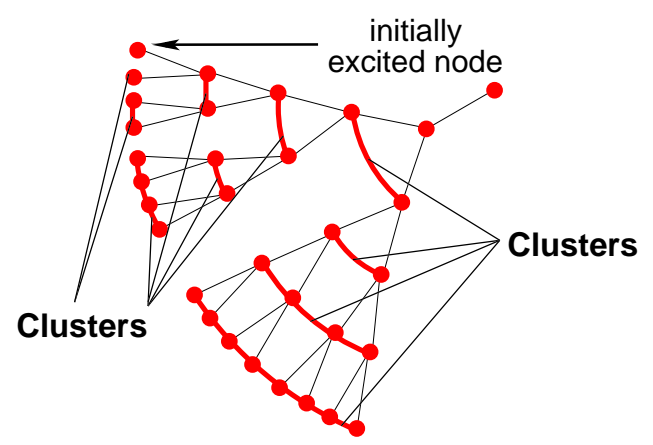

FIG. 13: (Color online). Clusters of the same limiting probability $\chi_{k, j}$ in the branch with the initial excitation for a dendrimer of generation $G$. Nodes connected by thick (red) lines belong to the same cluster. From [82].

i.e. $\lim _{t \rightarrow \infty} p_{k, j}(t)=1 / N$ for all nodes. Quantum mechanically this is not the case. Figure 12 shows the LPs $\chi_{k, j}$ as a contour plot [82]. Bright shadings correspond to high values of the LP, whereas dark shadings correspond to low LPs. The diagonal has high values, meaning that an excitation starting at node $j$ has a high LP to be found again at node $j$. The structures of the LP distributions of dendrimers are self-similar, generation after generation.

Furthermore, different nodes $k$ and $l$ may have the same LP, $\chi_{k, j}=\chi_{l, j}$. One hence combines all the nodes having (up to our numerical precision, $10^{-10}$ ) the same LP into a cluster. Note, however, that the separation of the nodes into clusters depends on the initially excited node, namely on $j$. For an excitation starting at the center (node 1), the clusters correspond exactly to the different generations of the dendrimer. In the general case, when starting from a non-central node, one still finds from Fig. 12 that nodes belonging to the same cluster also belong to the same generation (the converse is not necessarily true).

For larger dendrimers, while the general cluster pattern is preserved, some details change. Figure 13 shows the situation for a dendrimer of dimension $G=5$, for an excitation starting at a peripheral node. Again one indicates clusters by connecting nodes by thick (red) lines. A change to be noticed is that for $G \geq 5$ the initially excited node does not form anymore a cluster with its next-nearest node of the same generation. It appears as if such two nodes only belong to the same cluster when the dendrimer has $G \leq 4$. Thus the total number of clusters is $N_{C} \equiv\left(G^{2}+G+6\right) / 2$ for $G \geq 5$ and $N_{C}-1$ for $G \leq 4$.

\section{Husimi-cacti}

As shown in [87], it may happen that the excitation occupies preferentially the bonds between the branching points of the dendrimer. Then, the essential underlying structure is different and is given by sites localized at the mid-points of the bonds. The situation is exemplified in Fig.14(a), starting from a dendrimer of generation 2 (open circles) and indicating the mid-points of the bonds by filled circles. Connecting neigh- 


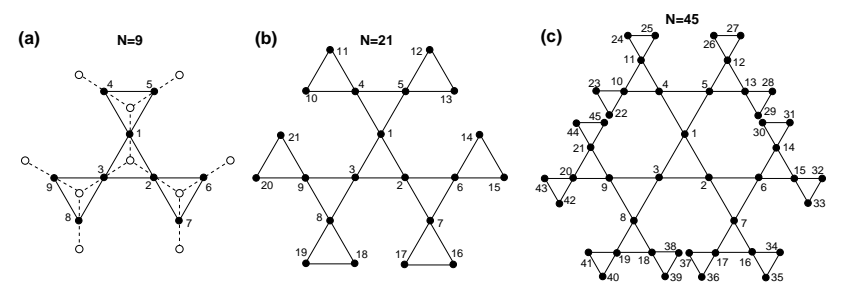

FIG. 14: Finite Husimi cacti (filled circles) of size (a) $N=9$, (b) $N=21$, and (c) $N=45$. (a) also shows with dashed lines and open circles the corresponding dendrimer. From [88].

(a) $\mathrm{N}=21$

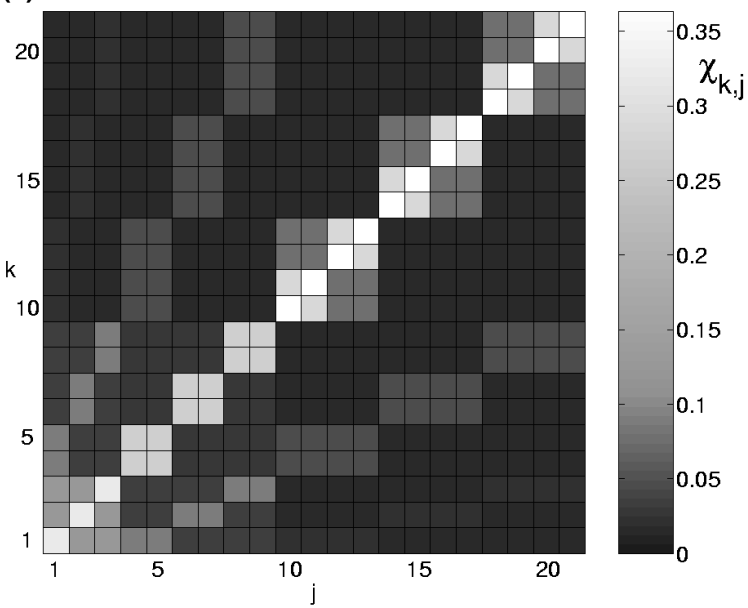

(b) $\mathrm{N}=45$

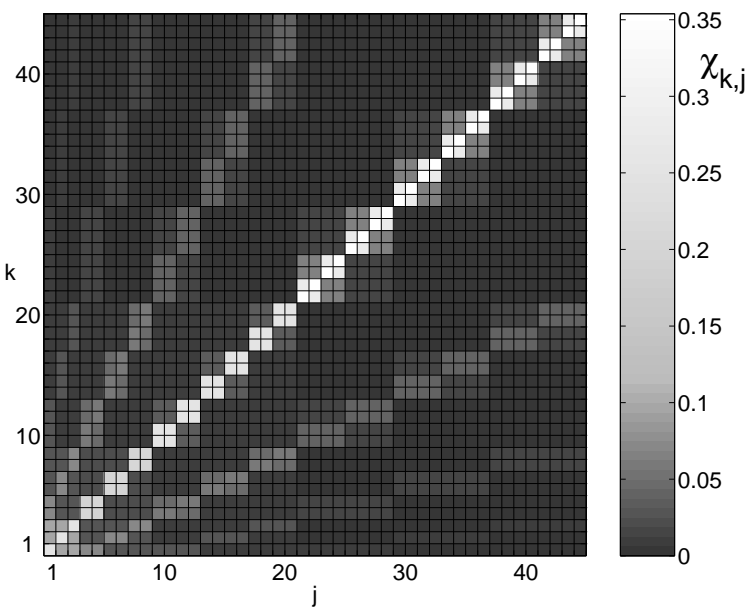

FIG. 15: Limiting probabilites for finite Husimi cacti of sizes (a) $N=21$ and (b) $N=45$. From [88].

boring filled circles by new bonds, one is led to the so-called Husimi cactus. Figure 14 shows three finite Husimi cacti of sizes $N=9,21$, and 45 .

In the same way as for the dendrimer, one finds numerically that for the finite Husimi cactus consisting of 21 nodes, as depicted in Fig. 14, the TPs are nearly periodic when the initial excitation is placed on one of the (symmetrically equivalent) nodes 1,2 , or 3 of the inner triangle [88].

Also in the long time limit, the similarities to the dendrimer

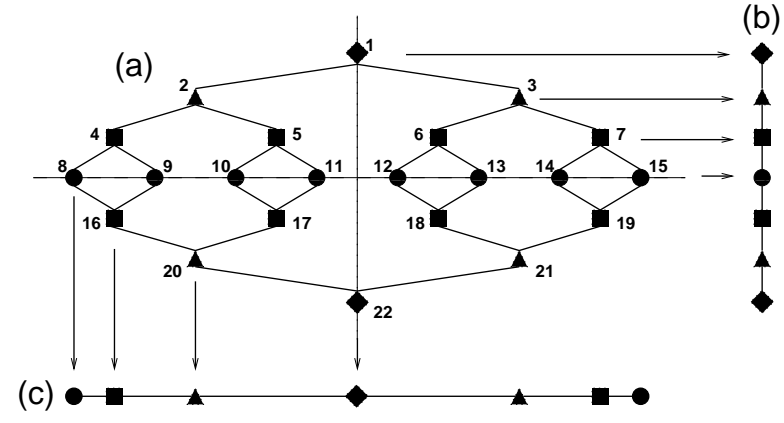

(d)

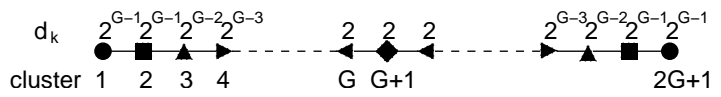

FIG. 16: (a) Graph consisting of two Cayley trees of generation $G=3$. (b) horizontal projection of the graph following Ref. [89], (c) vertical projection of the same graph. (d) Vertical projection of a similar graph, obtained from two Cayley trees of general generation $G$, indicating the new nodes (clusters) and the $d_{k}$, see text for details. From [23].

are obvious. Figure 15 presents the LPs for two sizes of Husimi cacti, $N=21$ and $N=45$. The LP distributions are self-similar generation after generation. Furthermore, for each size there are LPs having the same value, i.e. $\chi_{k, j}=\chi_{l, j}$. One collects LPs of the same value into clusters. Depending on where the excitation starts, the clustering is different. This in analogous to the previous results for dendrimers. Since both dendrimers and Husimi cacti lead to similar results, one can conclude that here the loops have no significant effect on the transition probabilities.

\section{Glued Cayley trees}

One particular example which shares the properties of both regular and hyperbranched networks is a network which is composed of two Cayley-trees with the same number of generations, where the nodes of the last generation are shared by both trees, see Fig. 16 .

Now, depending on the initial condition, the dynamics of the CTQW over such a network can change dramatically. While the transport from the top to the bottom node is very fast [89] and comparable to the dynamics on a finite regular one-dimensional network, the transport from the left-most node to the right-most node is very slow [23] compared to the transport in dendrimers or Husimi cacti when the excitation starts at a peripheral node [82, 88].

The authors of Refs. [21] and [89] have analyzed CTQW over such networks, focussing on walks which start at the top node, and looking for the amplitude of being at the bottom node at time $t$. The problem can then be simplified by considering only states which are totally symmetric superpositions of states $|k\rangle$, involving all the nodes $k$ in each row of Fig.16(a), as indicated schematically in Fig. 16(b). The transport gets then mapped onto a one-dimensional CTQW [89]. 
Other initial conditions for the CTQW are, indeed, possible, especially when considering the high symmetry of the underlying graphs. Note that, using for instance the site enumeration of Fig. 16 (a), a CTQW from node 8 to node 15 is equivalent to a CTQW from, say, node 10 to node 14 . The graph's symmetry suggests to collect groups of such nodes into clusters, while focussing on the transport from left to right. It is then natural to view the nodes $8,9,10$, and 11 as belonging to the first cluster. The second cluster consists then of the nodes $4,5,16$, and 17 , all of which are directly connected by one bond to the nodes of the first cluster. The nodes 2 and 20 of the third cluster are all nodes directly connected by one bond to the nodes of the second cluster, while at the same time not belonging to the first cluster. In general, all the nodes of the $(k+1)$ st cluster are connected by one bond to nodes of the $k$ th cluster and at the same time do not belong to the $(k-1)$ st cluster.

Let us denote the number of nodes in cluster $k$ by $d_{k}$. The transport occurs now from a cluster to the next, by which the original graph gets mapped onto a line in which one new node corresponds to a group of original nodes of the graph. For a new node at position $k \in[2, G]$ one finds that $d_{k}=2^{G-k+1}$, the same being true for the mirror node value, i.e., $d_{k}=$ $d_{2 G+2-k}$. Note that for the end nodes $d_{1}=d_{2 G+1}=2^{G-1}$, the same holds for the nodes next to them. Moreover, for the middle node $d_{G+1}=2$.

One now focuses on the transport via the states which are totally symmetric, normalized, linear state-combinations for all the original nodes in each cluster. Thus, for the $k$ th cluster, whose sites are denoted by $n$, one has as a new state [23]

$$
\left|a_{k}\right\rangle=\frac{1}{\sqrt{d_{k}}} \sum_{n \in k}|n\rangle
$$

The CTQW is now determined by the new Hamiltonian $\tilde{\boldsymbol{H}}=\gamma \tilde{\boldsymbol{A}}$, where the matrix elements of $\tilde{\boldsymbol{A}}$ are obtained from the new basis states $\left|a_{k}\right\rangle$ and from the matrix $\boldsymbol{A}$ through

$$
\tilde{A}_{j k}=\left\langle a_{j}|\boldsymbol{A}| a_{k}\right\rangle .
$$

Given the properties of $\boldsymbol{A}$ and the construction of the $\left|a_{k}\right\rangle$, Eq. (66), $\tilde{A}$ is a real and symmetrical tridiagonal matrix, which implies a CTQW on a line. The diagonal elements of $\tilde{\boldsymbol{A}}$ are given by

$$
\begin{aligned}
\tilde{A}_{k k} & =\left\langle a_{k}|\boldsymbol{A}| a_{k}\right\rangle \\
& =\frac{1}{d_{k}} \sum_{\substack{n \in k \\
n^{\prime} \in k}}\left\langle n^{\prime}|\boldsymbol{A}| n\right\rangle=f_{n} \equiv f_{k},
\end{aligned}
$$

where $f_{k}$ is the functionality of every node in the $k$ th cluster. For the sub- and super-diagonal elements of $\tilde{\boldsymbol{A}}$ one finds [23]

$$
\begin{aligned}
\tilde{A}_{k, k+1} & =\tilde{A}_{k+1, k}=\left\langle a_{k}|\boldsymbol{A}| a_{k+1}\right\rangle \\
& =\frac{1}{\sqrt{d_{k} d_{k+1}}} \sum_{\substack{n \in k \\
n^{\prime} \in k+1}}\left\langle n^{\prime}|\boldsymbol{A}| n\right\rangle \\
& =-\frac{b_{k}}{\sqrt{d_{k} d_{k+1}}},
\end{aligned}
$$
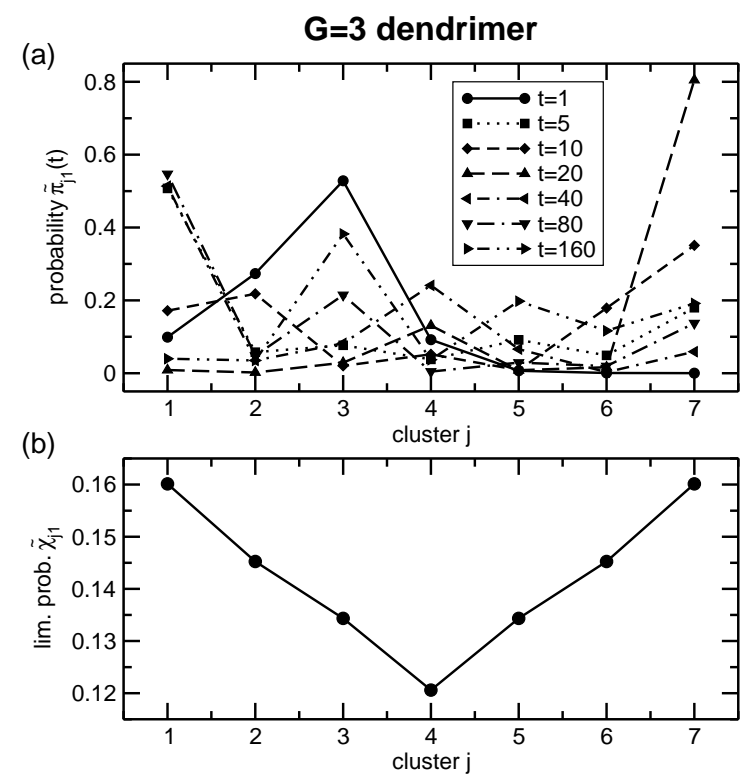

FIG. 17: (a) Transition probability $\tilde{\pi}_{j 1}(t)$ for a CTQW between different clusters $j$ of the $G=3$ graph. The CTQW starts at the first cluster, presented is the situation at times $t=1,5,10,20,40,80$, and 160 . (b) Limiting probability $\tilde{\chi}_{j 1}$ for a CTQW starting at the first cluster. From [23].

where $b_{k}$ is the number of bonds between the clusters $k$ and $k+1$.

Now, except for the ends and the center of the graph, $b_{k}$ equals the maximum of the pair $\left(d_{k}, d_{k+1}\right)$. Between the central node $\left(d_{G+1}=2\right)$ and its neighbors $\left(d_{G}=d_{G+2}=2\right)$ the number of bonds is $b_{G}=b_{G+2}=2$. The number of bonds between the end node and its neighbor is $b_{1}=b_{2 G+1}=2 d_{1}=$ $2^{G}$.

For the graph consisting of 22 original nodes the new matrix $\tilde{\boldsymbol{A}}$ is a tridiagonal $7 \times 7$ matrix, which can be readily diagonalized. The advantage of the procedure is clear: the new matrix $\tilde{A}$ depends on the number of clusters and grows with $(2 G+1)$, whereas the full adjacency matrix, $\boldsymbol{A}$, grows with the total number of nodes in the graph, namely with $\left(3 \cdot 2^{G}-2\right)$.

From Eq. (66) the transition amplitude between the state $\left|a_{k}\right\rangle$ at time 0 and the state $\left|a_{j}\right\rangle$ at time $t$ is given by [23]

$$
\tilde{\alpha}_{j k}(t)=\left\langle a_{j}\left|e^{-i \tilde{\boldsymbol{H}} t}\right| a_{k}\right\rangle=\left\langle a_{j}\left|\tilde{\boldsymbol{Q}} e^{-i \gamma \tilde{\boldsymbol{\Lambda}} t} \tilde{\boldsymbol{Q}}^{-1}\right| a_{k}\right\rangle,
$$

where $\tilde{\Lambda}$ is the eigenvalue matrix and $\tilde{Q}$ the matrix constructed from the orthonormalized eigenvectors of the new matrix $\tilde{A}$.

Now the quantum mechanical transition probabilities are given by $\tilde{\pi}_{j k}(t)=\left|\tilde{\alpha}_{j k}(t)\right|^{2}$. Figure 17(a) shows the transition probabilities for CTQW over clusters. Remarkably, now already during short periods of time, such CTQW move from one end cluster to the other one. The limiting probability distribution, $\tilde{\chi}_{j k}$, which is depicted in Fig. 17(b), also supports this finding. Note that Fig. 17 (b) again reflects the symmetry of the original graph. 


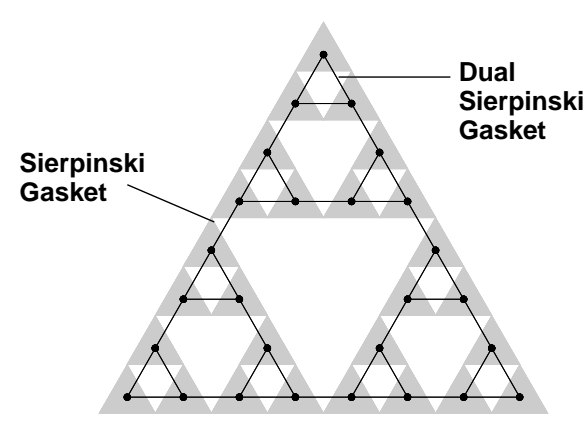

FIG. 18: Dual transformation from Sierpinski Gasket to Dual Sierpinski Gasket of generation $g=3$. From [90].

\section{B. Fractals}

\section{Sierpinski Gaskets}

Regular networks have integer dimensions, while some tree-like stuctures presented in the previous section, such as the dendrimers, can be of "infinite fractal dimension", when the (fractal) dimension is taken to be given by $d_{f}=$ $\lim _{R \rightarrow \infty} \ln N / \ln R, N$ being the number of nodes within a sphere of radius $R$. . In contrast, (deterministic) fractals have finite, in general, non-integer dimensions. One particular example of a deterministic fractal is the dual Sierpinski gasket (DSG) for which the exact spectrum of the eigenvalues of the connectivity matrix is known [90]. A DSG is an exactly-decimable fractal which is directly related, through a dual transformation, to the Sierpinski gasket (SG). The DSG of generation $g$ can be constructed by replacing each small triangle belonging to the SG with a node and by connecting such nodes whenever the relevant triangles share a vertex in the original gasket (see Fig. 18). It is straightforward to verify that the number of nodes at any given generation $g$ is $N=3^{g}$.

The dual transformation does not conserve the coordination number (which decreases from 4 to 3 , while the coordination number of nodes corresponding to the vertices of the gasket remains 2), but it does conserve the fractal dimension $d_{f}$ and the spectral dimension $\tilde{d}$, which are therefore the same as for the original Sierpinski gasket, namely $d_{f}=\ln 3 / \ln 2=$ $1.58496 \ldots$ and $\tilde{d}=2 \ln 3 / \ln 5=1.36521 \ldots$. The eigenvalue spectrum of the DSG connectivity matrix can be determined at any generation through the following iterative procedure (for more details see [91, 92]): At any given generation $g$ the spectrum includes the non-degenerate eigenvalue $\lambda_{N}=0$, the eigenvalue 3 with degeneracy $\left(3^{g-1}+3\right) / 2$ and the eigenvalue 5 with degeneracy $\left(3^{g-1}-1\right) / 2$. Moreover, given the eigenvalue spectrum at generation $g-1$, then to each non-vanishing eigenvalue $\lambda_{g-1}$ correspond two new eigenvalues $\lambda_{g}^{ \pm}$accord-

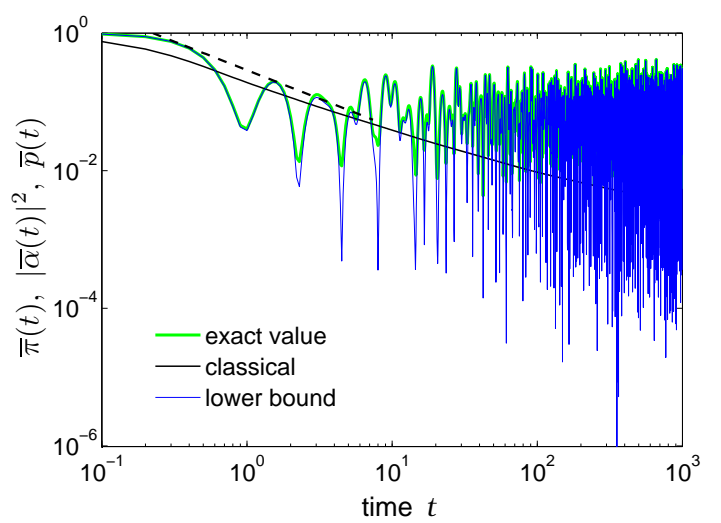

FIG. 19: (Color online) Average return probability $\bar{\pi}(t)$ for the DSG of generation $g=5$ on a log-log scale. The comparison with the classical $\bar{p}(t)$ evidences that the classical random walk spreads more efficiently than its quantum-mechanical counterpart. The dashed line represents the envelope of $\bar{\pi}(t)$. From [90].

ing to

$$
\lambda_{g}^{ \pm}=\frac{5 \pm \sqrt{25-4 \lambda_{g-1}}}{2} ;
$$

both $\lambda_{g}^{+}$and $\lambda_{g}^{-}$inherit the degeneracy of $\lambda_{g-1}$. The eigenvalue spectrum is therefore bounded in $[0,5]$. As explained in [91], at any generation $g$, one can calculate the degeneracy of each distinct eigenvalue: apart from $\lambda_{N}$ whose degeneracy is 1 , there are $2^{r}$ distinct eigenvalues, each with degeneracy $\left(3^{g-r-1}+3\right) / 2$, being $r=0,1, \ldots, g-1$, and $2^{r}$ distinct eigenvalues, each with degeneracy $\left(3^{g-r-1}-1\right) / 2$, being $r=0,1, \ldots, g-2$. As can be easily verified, the degeneracies sum up to $N=3^{g}$.

For the DSG the CTRW average return probability $\bar{p}(t)$ is readily obtained without numerically diagonalizing the connectivity matrix, since it only depends on the eigenvalues which can be calculated iteratively. Figure 19 displays the averaged probabilities $\bar{p}(t), \bar{\pi}(t)$ and $|\bar{\alpha}(t)|^{2}$ as a function of time, obtained for $g=5$. The classical $\bar{p}(t)$ decays monotonically to the equipartition value $1 / N$, while the quantummechanical probabilities eventually oscillate around the value 0.7 , which is larger than $3^{-g}$. Although the amplitude of fluctuations exhibited by the lower bound is larger than that of the exact value, the agreement between the two quantities is very good. In particular, the positions of the extremal points practically coincide and the maxima of $\bar{\pi}(t)$ are well reproduced by the lower bound. This is analogous to the behavior of walks on square lattices, Cayley trees, and stars, as described in the previous sections. Notice, however, that for the square lattices the lower bound turns out to be exact while for Cayley trees and for stars it is only an approximation, which, moreover, turns out to be less accurate than what is found for the DSG.

At short times $\left(t<5 \gamma^{-1}\right)$ it is possible to construct the envelope of $\bar{\pi}(t)$, which depends algebraically on $t$ [90]. The exponent is $\approx-0.82$, to be possibly compared with $\tilde{d} / 2 \approx$ -0.68 which is the exponent expected classically for the infinite DSG. The decay of the average return probability $\bar{\pi}(t)$ 


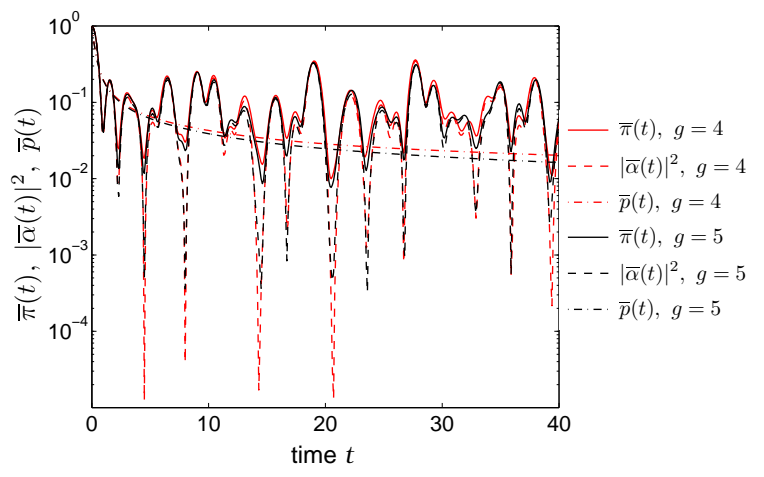

FIG. 20: (Color online) Average return probability $\bar{\pi}(t)$ for the DSG of generation $g=4$ (bright colour) and $g=5$ (dark colour). Its lower bound $|\bar{\alpha}(t)|^{2}$ (dashed line) and the classical $\bar{p}(t)$ (dotted line) are also depicted, as shown by the legend. From [90].

for the ST can be estimated as well: its envelope goes like $t^{-2}$ (classically $\bar{p}(t) \sim t^{-1}$, see above), implying a faster delocalization of the $\mathrm{QW}$ over the graph.

Interestingly, for the DSG, the overall shape of $\bar{\pi}(t)$ does not depend significantly on the size of the gasket (see Fig. 20 and [90]). In fact, the behaviour of $\bar{\pi}(t)$ is mainly controlled by the most highly degenerate eigenvalues. These do not change when increasing the fractal size (i.e. its generation). These values are: 3 with degeneracy $m_{g}(3)=\left(3^{g-1}+3\right) / 2$, 5 with degeneracy $m_{g}(5)=\left(3^{g-1}-1\right) / 2,(5 \pm \sqrt{13}) / 2$ with degeneracy $m_{g-1}(3)$.

The inhomogeneity of the pattern of the long-time averages $\chi_{k, j}$ mirrors the lack of translation invariance of the DSG itself. For instance, being $v$ the label assigned to any vertex of the main triangle, $\chi_{v, v}$ is a global maximum; off-diagonal local maxima correspond to couples of connected nodes belonging to different minor triangles of generation $g-1$. This allows to establish a mapping between the pattern of $\chi_{k, j}$ and the structure of the relevant DSG.

Since the spectrum of the DSG is known, one can calculate the lower bound of the long-time average of the average return probability, $\bar{\chi}_{l b}$, analytically. At generation $g$ the spectrum of the connectivity matrix displays $N$ distinct eigenvalues, where

$$
\tilde{N}=\sum_{r=0}^{g-1} 2^{r}+\sum_{r=0}^{g-2} 2^{r}+1=3 \times 2^{g-1}-1 .
$$

Let us denote the set of distinct eigenvalues by $\left\{\tilde{\lambda}_{i}\right\}_{i=1, \ldots, \tilde{N}}$. Being $m\left(\lambda_{i}\right)$ the degeneracy of the eigenvalue $\lambda_{i}$, one can write

$$
N^{2} \bar{\chi}_{l b}=\sum_{n, m=1}^{\mathcal{N}} \delta_{\lambda_{n}, \lambda_{m}}=\sum_{n=1}^{N} m\left(\lambda_{n}\right)=\sum_{i=1}^{\tilde{N}}\left[m\left(\tilde{\lambda}_{i}\right)\right]^{2} .
$$

Now, one goes over to the space of distinct degeneracies, each corresponding to a number $\rho$ of distinct eigenvalues and gets (a) $g=3$

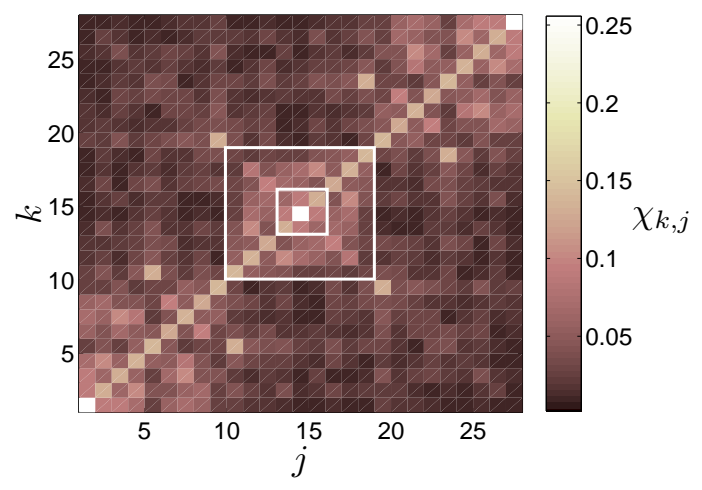

(b) $g=4$

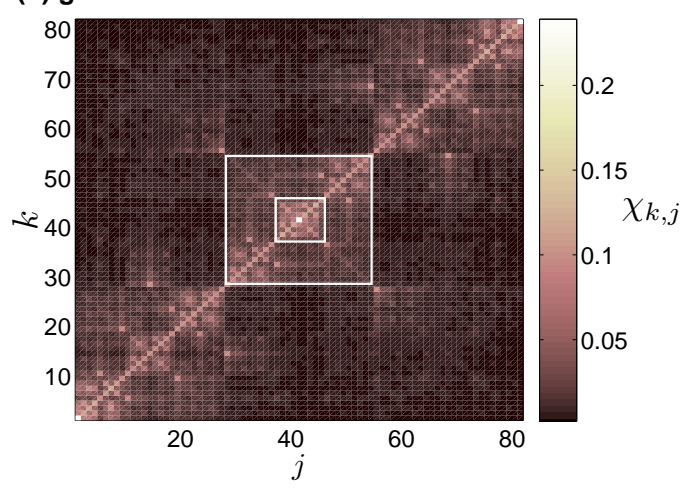

FIG. 21: Limiting probabilities for the DSG of generation (a) $g=3$ and (b) $g=4$, whose volumes are $N=27$ and $N=81$, respectively. The white lines enclose the limiting distributions for gaskets of smaller generations. Notice that the global maxima lay on the main diagonal and correspond to $j=1,14,27$ and to $j=1,41,81$ for $g=3$ and for $g=4$, respectively. From [90].

the final, explicit formula [90]

$$
\begin{aligned}
\bar{\chi} \geq & \bar{\chi}_{l b}=\frac{1}{N^{2}} \sum_{r=0}^{2 g}[m(r)]^{2} \rho(m(r)) \\
= & \frac{1}{N^{2}}\left\{\sum_{r=0}^{g-1}\left[\frac{3^{g-r-1}+3}{2}\right]^{2} \times 2^{r}\right. \\
& \left.+\sum_{r=0}^{g-2}\left[\frac{3^{g-r-1}-1}{2}\right]^{2} \times 2^{r}+1\right\} \\
= & \frac{1}{3^{2 g}}\left[3^{g}\left(1+\frac{3^{g}}{14}\right)+\frac{10}{7} 2^{g}-\frac{3}{2}\right],
\end{aligned}
$$

such that $\bar{\chi}>1 / 3^{g}$. Interestingly, in the limit $g \rightarrow \infty$, the LTA $\bar{\chi}$ is finite [90]:

$$
\bar{\chi} \geq \lim _{g \rightarrow \infty} \bar{\chi}_{l b}=\frac{1}{14},
$$

and $\bar{\chi}_{l b}$ reaches this asymptotic value from above. 


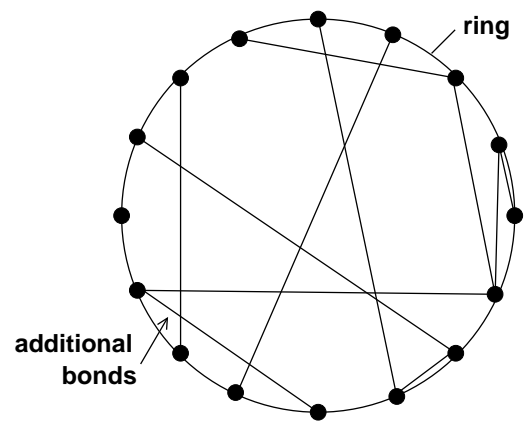

FIG. 22: Sketch of a SWN of size $N=16$ containing $B=11$ additional bonds. From [93].

\section{Statistical networks}

\section{Small-world networks}

The above examples dealt with deterministic networks. However, many real systems have stochastic features; thus the connectivity can be random. To model this, one can disrupt the periodicity of regular patterns by randomly including $B$ additional bonds into the network [93]. In such a way one creates "shortcuts" and a walker can find shorter paths between pairs of sites than on the regular network. So-called small-world-networks (SWN) are created by randomly adding bonds to a regular one dimensional ring, see Fig. 22 Here we do not consider self-connections, i.e., bonds connecting one node with itself.

The general behavior of CTQW on SWN can be analyzed by averaging over distinct realizations $R$

$$
\langle\cdots\rangle_{R} \equiv \frac{1}{R} \sum_{r=1}^{R}[\cdots]_{r},
$$

where the index $r$ specifies the $r$ th realization of the quantity in question. In so doing one obtains statistical results which allow for a comparison with the deterministic situation. In particular, we consider here the realization-averaged transition probabilities $\left\langle\pi_{k j}(t)\right\rangle_{R}$, the averaged probabilities $\langle\bar{\pi}(t)\rangle_{R}$, their lower bound $\langle\bar{\alpha}(t)\rangle_{R}$, and their classical analog $\langle\bar{p}(t)\rangle_{R}$. Furthermore, we also discuss the long time average (LTA) of each of these quantities:

$$
\left\langle\lim _{T \rightarrow \infty} \frac{1}{T} \int_{0}^{T} d t \cdots\right\rangle_{R} .
$$

In the absence of any additional bond, the excitations travel along the ring and interfere in a very regular manner, producing discrete quantum carpets [72]. Typical for these carpets is that they show, depending on $N$, full or partial revivals at specific times [72].

For SWN the situation is quite different. Already a few additional bonds obliterate the quantum carpets; the patterns fade away [93]. By adding more bonds, only the initial node retains a significant value for $\left\langle\pi_{j j}(t)\right\rangle_{R}$ at all times $t$. Furthermore, already for SWN with as little as $B=5$ the pattern of $\left\langle\pi_{j j}(t)\right\rangle_{R}$ becomes quite regular after a short time, see
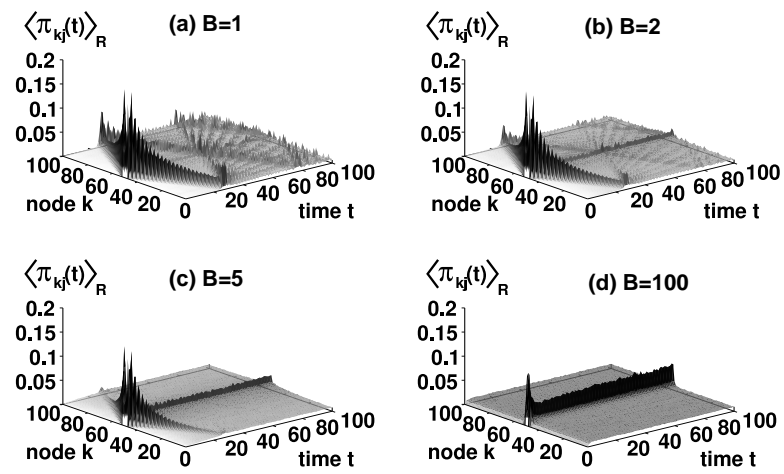

FIG. 23: Time dependence of the averaged transition probabilities $\left\langle\pi_{k j}(t)\right\rangle_{R}$ for SWN of size $N=100$ with (a) $B=1$, (b) $B=2$, (c) $B=5$, and (d) $B=100$. The initial node is $j=50$ and the number of realizations is $R=500$. From [93].

Fig. 23(c). This almost regular shape is reached very quickly when $B$ gets to be comparable to $N$ [Fig. 23(d)]. One notes, however, that particular realizations may still show (depending on their actual additional bonds) strong interference patterns. These features are washed out by the ensemble average, so that only the dependence on the initial node stands out.

Since CTQW on SWN always carry the information of their initial node $j$, the averaged probabilities to return to $j$ are a good measure to quantify the efficiency of the transport on such networks, see [73].

Figure 24 shows in double-logarithmic scales the ensemble averages $\langle\bar{p}(t)\rangle_{R},\langle\bar{\pi}(t)\rangle_{R}$, and $\langle\bar{\alpha}(t)\rangle_{R}$ for SWN with $N=100$ nodes and $B=1,2,5$, and 100 . For classical transport [Fig. 24(a)] the initial decay of $\langle\bar{p}(t)\rangle_{R}$ occurs faster for larger $B$. The decay at intermediate times follows a power-law $\left(t^{-1 / 2}\right)$ for the ring (as is clear from the linear behavior in the scales of the figure) and changes to a stretched exponentialtype when $B$ is large [94]. Thus, a classical excitation will quickly explore the whole SWN, so that it will occupy each site with equal probability of $1 / N$ already after a relatively short time, see the final plateau in Fig. 24 a).

Quantum mechanically, however, the situation is more complex. Fig. 24 (b) shows the ensemble average $\langle\bar{\pi}(t)\rangle_{R}$. For a ring of $N$ nodes and for times shorter than roughly $N / 2\langle\bar{\pi}(t)\rangle_{R}$ displays a quasiperiodic pattern (black curve), the maxima of which decay as $t^{-1}$. At longer times interference sets in and leads to an irregular behavior at times longer than $N / 2$ [73]. Now, for SWN, as long as $B$ is considerably less than $N$, the periodic pattern still remains visible; in Fig. 24(b) one can follow how an increase in $B$ (red, green, and blue curves) is smoothing out the curves, so that both the heights of the first maxima and the depths of the minima decrease. At longer times the SWN patterns are flattened out and $\langle\bar{\pi}(t)\rangle_{R}$ tends towards a limiting value. With increasing $B$ this asymptotic domain is reached more quickly, such that for larger $B$ the crossover from the quasiperiodic behavior at short times to a smoothed out pattern at longer times is shifted to smaller $t$.

Figure 24(c) shows the lower bound of $\bar{\pi}(t)$, namely $\left\langle|\bar{\alpha}(t)|^{2}\right\rangle_{R}$ averaged over the realizations. One notices that 

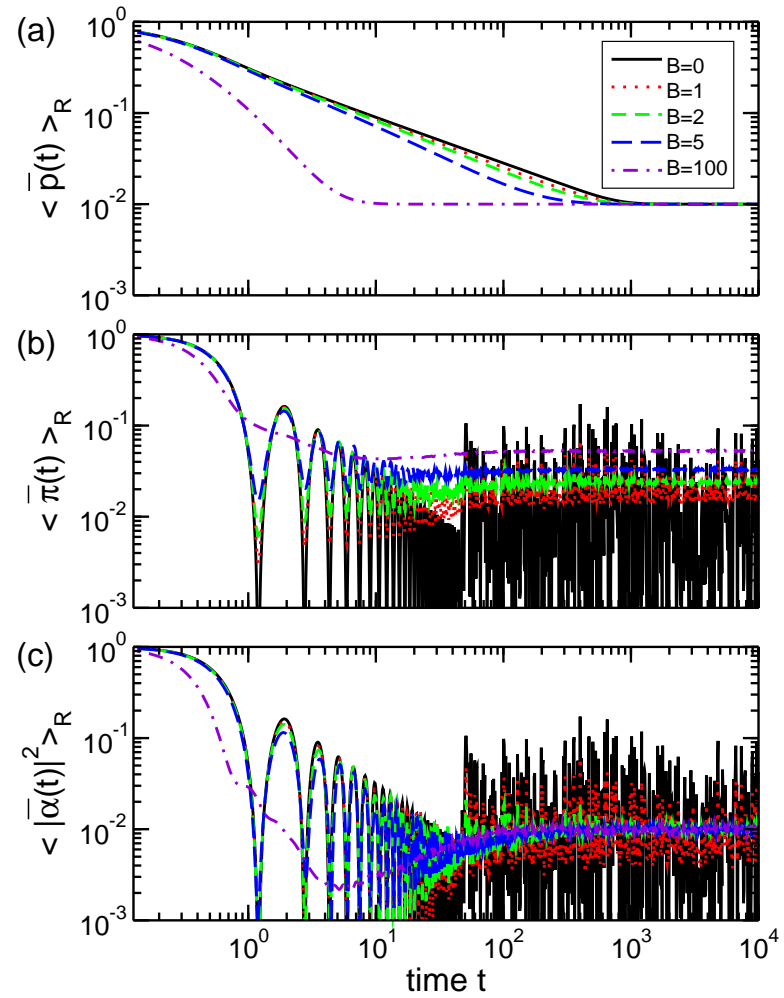

FIG. 24: (Color online) Time dependence of the averaged probabilities (a) $\langle\bar{p}(t)\rangle_{R}$, (b) $\langle\bar{\pi}(t)\rangle_{R}$, and (c) $\left\langle|\bar{\alpha}(t)|^{2}\right\rangle_{R}$ for SWN of size $N=100$ with $B=1,2,5$, and 100 . The number of realizations is $R=500$. From [93]

the overall behavior of Figs. 24 b) and 24(c) is quite similar. However, the limiting values at long times differ. For the LTA of $\langle\bar{\pi}(t)\rangle_{R}$ one has (see also Eq. (17) of Ref. [71])

$$
\begin{aligned}
\langle\bar{\chi}\rangle_{R} \equiv & \left\langle\lim _{T \rightarrow \infty} \frac{1}{T} \int_{0}^{T} d t \bar{\pi}(t)\right\rangle_{R} \\
= & \frac{1}{R N} \sum_{r, j, n, n^{\prime}} \delta\left(E_{n, r}-E_{n^{\prime}, r}\right) \\
& \times\left|\left\langle j \mid \Phi_{n, r}\right\rangle\left\langle j \mid \Phi_{n^{\prime}, r}\right\rangle\right|^{2}
\end{aligned}
$$

where $\delta\left(E_{n, r}-E_{n^{\prime}, r}\right)=1$ for $E_{n, r}=E_{n^{\prime}, r}$ and $\delta\left(E_{n, r}-\right.$ $\left.E_{n^{\prime}, r}\right)=0$ otherwise. For $\left\langle|\bar{\alpha}(t)|^{2}\right\rangle_{R}$ the long-time values for different $B$ collapse to one value. In fact, the LTA of $\left\langle|\bar{\alpha}(t)|^{2}\right\rangle_{R}$ obeys

$$
\begin{aligned}
& \left\langle\lim _{T \rightarrow \infty} \frac{1}{T} \int_{0}^{T} d t|\bar{\alpha}(t)|^{2}\right\rangle_{R} \\
& =\frac{1}{R N^{2}} \sum_{r, n, n^{\prime}} \delta\left(E_{n, r}-E_{n^{\prime}, r}\right)
\end{aligned}
$$

as can be immediately inferred from Eq. (24). Thus this quantity is only a function of the eigenvalues $E_{n, r}$ and does not depend on the eigenstates $\left|\Phi_{n, r}\right\rangle$. In order to quantify the differences between Eqs. (78) and (79) for SWN, one assumes that all the eigenvalues are nondegenerate (this assumption is, of course, not valid for the ring, see below). In Eq. (79) the triple sum adds then to $R N$, so that the rhs equals $1 / N$. On the other hand, Eq. (78) leads to [93]

$$
\langle\bar{\chi}\rangle_{R}=\frac{1}{R N} \sum_{r, j, n}\left|\left\langle j \mid \Phi_{n, r}\right\rangle\right|^{4} .
$$

This expression depends on the eigenstates; in fact the rhs of Eq. (80) is the ensemble average of the average participation ratio of the eigenstates $\left|\Phi_{n, r}\right\rangle$. Equation (80) is well known in the theory of quantum localization, see, e.g., Sec. V. A. in [95]. For the ring the eigenstates are Bloch states,

$$
\left|\Phi_{n}\right\rangle=\frac{1}{\sqrt{N}} \sum_{j=1}^{N} e^{i E_{n} j}|j\rangle,
$$

from which $\left|\left\langle k \mid \Phi_{n}\right\rangle\right|^{4}=1 / N^{2}$ follows for all $\left|\Phi_{n}\right\rangle$.

Now, increasing $B$ results in an increase of $\langle\bar{\chi}\rangle_{R}$, starting from the corresponding value for the $\operatorname{ring}(B=0$, only one realization, and $N$ even)

$$
\left\langle\bar{\chi}_{\text {ring }}\right\rangle_{R} \equiv \bar{\chi}=\frac{1}{N} \sum_{j} \chi_{j j}=\frac{2 N-2}{N^{2}},
$$

where $\chi_{j j}=(2 N-2) / N^{2}$. Equation (79) yields a $1 / N$ dependence for the LTA of $\left\langle|\bar{\alpha}(t)|^{2}\right\rangle_{R}$, which by rescaling with $\left\langle\bar{\chi}_{\text {ring }}\right\rangle_{R} \sim 1 / N$ would result in a constant value for large $N$ [93]. However, rescaling $\langle\bar{\chi}\rangle_{R}$ with $\left\langle\bar{\chi}_{\text {ring }}\right\rangle_{R}$ shows an increase with $N$ of $\langle\bar{\chi}\rangle_{R} /\left\langle\bar{\chi}_{\text {ring }}\right\rangle_{R}$ which is less than linear, thus, $\langle\bar{\chi}\rangle_{R}$ depends on $N$ as $1 / N^{\nu}$, with $\nu \in[1,2]$.

The fact that $\langle\bar{\chi}\rangle_{R}$ for SWN increases with increasing $B$ points towards a change of $\left|\left\langle k \mid \Phi_{n}\right\rangle\right|^{4}$ from the value $1 / N^{2}$. The situation may be visualized as follows: For the ring all eigenstates are Bloch states and hence are completely delocalized. Going over to SWN and increasing the number of additional bonds $B$ leads to localized states at the band edges and to fairly delocalized states well inside the band. The increase of $\langle\bar{\chi}\rangle_{R}$ is thus mainly due to the localized band edge states.

\section{Erdös-Rényi networks}

Somewhat similar to the SWN is the Erdös-Rényi network (ERN). One starts with $N$ disconnected nodes, every pair of nodes is then connected with the probability $p$, where only single connections between two nodes are allowed. One can in turn associate to $p$ an average degree $\bar{k}$ of the nodes, which is related to $p$ by $\bar{k}=p(N-1)$. For large network sizes $N$, the degree distribution $P(k)$ of the ERN is Poissonian peaked at $\bar{k}$.

$\mathrm{Xu}$ and Liu showed in Ref. [96] that in the ensemble average, the average return probabilities display a behavior very similar to the SWN, see Sec. IV C 1] and [93]. Although the probabilities decay, they do so only until they reach a plateau (for $N=100$ ), at a level considerably higher than the equipartition value of $1 / N$. The behavior is only weakly affected by the value of the average degree $\bar{k}$. 


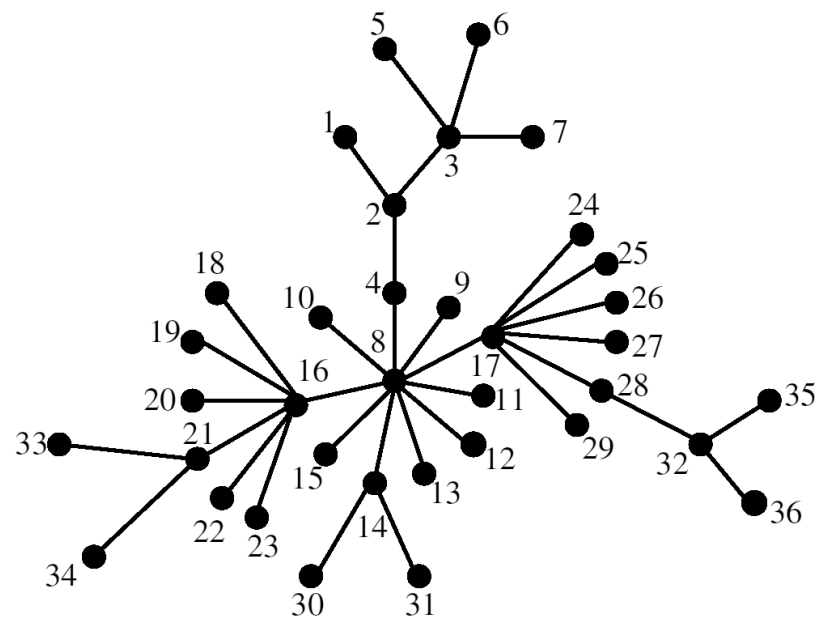

FIG. 25: Example of a scale-free network with $N=36$ nodes and $\phi=5 / 2$.

The height of the plateau is determined by the long-time behavior of the transition probabilities. Due to the ensemble average, all structure of the non-diagonal elements of $\left\langle\Xi_{k, j}\right\rangle$ disappears and only the main diagonal $\left\langle\Xi_{j, j}\right\rangle$ remains.

\section{Scale-free networks}

The distribution $P(k)$ for the number $k$ of bonds emanating from a node does not need to be Poissonian in general. Networks for which the distribution $P(k)$ follows a power-law, i.e., $P(k) \sim k^{-\phi}$, are called scale-free networks (SFN), see Fig. 25. These have been proven useful in various fields of research from biology to social sciences. $\mathrm{Xu}$ and Liu have considered CTQW over such structures [97]. They distinguish between deterministic scale-free networks (DSFN) and random scale-free networks (RSFN): the latter may obey distinct building procedures which can lead to either tree-like structures or structures containing loops.

For the DSFN, $\mathrm{Xu}$ and Liu determined the return probablities $\pi_{j, j}(t)$ and long-time averages $\chi_{k, j}$. They find that there is a strong dependence of $\pi_{j, j}(t)$ on the initial node $j$, which can even result in (almost) complete revivals of the initial condition. The transition probabilities translate directly into the long-time averages $\chi_{k, j}$, where one can identify clusters of nodes having the same $\chi_{k, j}$. However, the patterns obtained are quite distinct from the previously found patterns for the dendrimers [82] or for the Husimi cacti [88]. As mentioned earlier, this is a direct consequence of the fact that the $\chi_{k, j}$ mirror the topology of the network.

For RSFN, $\mathrm{Xu}$ and Liu calculated the ensemble average over many realizations of individual RSFN [97]. In the ensemble average the return probabilities do not oscillate but rather reach a stationary value, which differs for different initial nodes. In all cases, however, this stationary value is roughly one order of magnitude larger than the classical equipartition value $1 / N$ obtained for CTRW. Moreover, the higher the symmetry of the initial node (where on average the central nodes

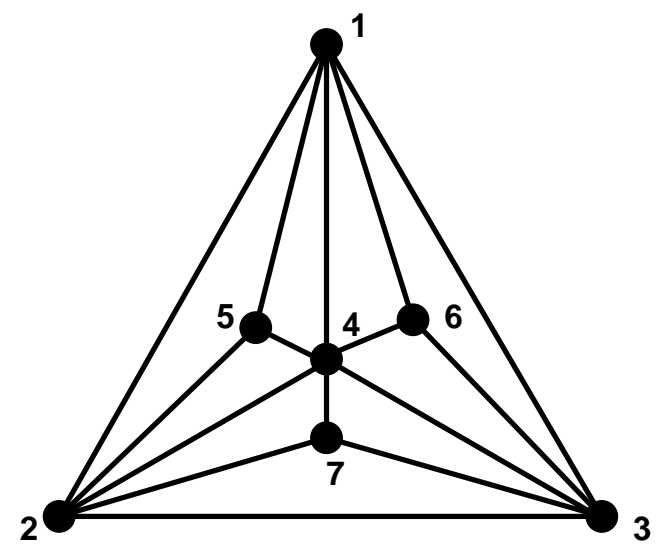

FIG. 26: Example of an apollonian network of generation $G=2$.

have the largest symmetry), the larger is the stationary value. Large average return probablities are also found in the longtime averages $\chi_{k, j}$, where in the ensemble average the values on the diagonal (for $k=j$ ) are much larger than the values for $k \neq j$.

\section{Apollonian networks}

So-called Apollonian networks are models for networks which have small-world as well as scale-free properties. CTQW on such structures have been investigated by $\mathrm{Xu}$ et al. [98]. The network is generated from a triangle $(N=3$ at generation $g=0$ ). In generation $g=1$ a single node is added which is connected with all three nodes of $g=0$, this divides the triangle of $g=0$ into three distinct triangles. In $g=2$ three new noded are added which are placed at the center of each of the three triangles of $g=1$, see Fig. 26. These three nodes divide each triangle of $g=1$ into three new triangles, such that there are now nine new triangles in total. The iteration proceeds in the same manner, such that in generation $G$ the total number of nodes is $N=3+\left(3^{G}-1\right) / 2$.

For $G=1$ the Apollonian network is identical to the complete graph of size $N=4$, therefore the transition probabilities for CTQW are fully periodic. It also turns out that for $G=2(N=7)$, see Fig. 26, and when choosing as the initial node the central, most symmetric node 4 , the transition probabilities are also fully periodic, being namely [97]

$$
\pi_{k, 4}(t)= \begin{cases}{[37+12 \cos (7 t)] / 49} & \text { for } k=4 \\ {[2-2 \cos (7 t)] / 49} & \text { for } k \neq 4\end{cases}
$$

However, also here $\mathrm{Xu}$ et al. observe a strong dependence on the initial condition. If the initial node is not a central node being, say, node 1 , the transition probabilities $\pi_{1,1}(t)$ are still periodic, although there is no perfect revival. Enlarging the networks lets the strong dependence on the initial condition be more pronounced. But, similar to the dendrimers [82], Xu et al. also identify clusters of nodes which, in the long-time average, have the same value for their probabilities $\chi_{k, j}$. 


\section{EXTENSIONS}

\section{A. Systems with long-range interactions}

So far the Hamiltonian for CTQW and the transfer matrix for CTRW have been directly related to the connectivity matrix. However, this matrix is purely topological (indicating whether or not two nodes are connected) and does not take metric (i.e., distance dependent) aspects into account. Some adjustment is thus necessary if one considers distancedependent interactions. As an example, consider the network being embedded in the $d$-dimensional space; then to every node $j$ one associates a vector $j$ whose coordinates are $\left\{x_{j 1}, \ldots, x_{j d}\right\}$. The distance between two nodes $j$ and $k$ is then given by the euclidean norm $R \equiv|\boldsymbol{k}-\boldsymbol{j}|$. Consider now systems in which the interaction between the two nodes $j$ and $k$ decreases with increasing distance. An example for such a system could be a collection of dipoles, interacting via dipole-dipole forces, whose potential decreases, to a good approximation, as $R^{-3}$. Now, in terms of the connectivity matrix, a node $j$ is not only connected to its nearest neighbors but also to other nodes. Thus, due to the decaying interaction potential the transition rates are not the same for all bonds but they depend on the distance.

Take as a first example a one-dimensional network with periodic boundary conditions (i.e., a discrete ring) [99]. Here, when the interactions go as $R^{-\gamma}$, the Hamiltonian has the following structure:

$$
\boldsymbol{H}_{\gamma}=\sum_{n=1}^{N} \sum_{R=1}^{R_{\max }} R^{-\gamma}(2|n\rangle\langle n|-| n-R\rangle\langle n|-| n+R\rangle\langle n|),
$$

where $R_{\max }$ is a cut-off for finite systems. Note, that in the infinite system limit one first takes $N \rightarrow \infty$ before taking also $R_{\max } \rightarrow \infty$. For the cases considered here, namely $\gamma \geq 2$ and $N$ of the order of a few hundred nodes, a resonable cut-off is $R_{\max }=N / 2$, which is also the largest distance between two nodes on the discrete ring. In this way, to each pair of sites a single (minimal) distance and a unique interaction is assigned.

For all $\gamma$, the eigenstates are again the Bloch states $\left|\Psi_{\theta}\right\rangle$ given above. The fact that the eigenstates are not affected by the long-range interactions is due to the translational invariance along the ring. For other systems without such an invariance the eigenstates will also change depending on the type of the interaction. From the eigenstates one obtains the eigenvalues which now do depend on the interaction range [99]:

$$
E_{\gamma}(\theta)=\sum_{R=1}^{R_{\max }} R^{-\gamma}[2-2 \cos (\theta R)]
$$

The DOS $\rho_{\gamma}(E)$ is obtained by inverting Eq. (85) and taking the derivative with respect to $E_{\gamma}$. In the NN-case $(\gamma=\infty)$ one gets the known DOS

$$
\rho_{\infty}(E)=\left(\pi \sqrt{4 E-E^{2}}\right)^{-1}
$$

For $\gamma=2$ one can approximate the sum by letting $R_{\max } \rightarrow$ $\infty$, which yields $E_{2}(\theta)=\pi \theta-\theta^{2} / 2$, and one obtains

$$
\rho_{2}(E)=\left(\pi \sqrt{2} \sqrt{\pi^{2} / 2-E}\right)^{-1} .
$$

In the intermediate range there is an analytic solution for $\gamma=$ 4, namely $E_{4}(\theta)=\theta^{4} / 24-\pi \theta^{3} / 6+\pi^{2} \theta^{2} / 6$ (see Eq. 1.443 .6 of [75]), which yields [100]

$$
\rho_{4}(E)=\left[2 \pi(2 / 3)^{1 / 4} \sqrt{E\left(\pi^{2} / \sqrt{24}\right)-E^{3 / 2}}\right]^{-1} .
$$

One assumes the following general form for the DOS in order to interpolate between $\rho_{2}(E)$ and $\rho_{\infty}(E)$ [99]:

$$
\rho_{\gamma}(E) \sim\left[\sqrt{c_{\gamma} E^{\alpha}-E^{\beta}}\right]^{-1}
$$

with $\alpha \in[0,1]$ and $\beta \in[1,2] ; c_{\gamma}$ is a constant related to the maximal energy, $c_{\gamma} \equiv\left(E_{\gamma, \max }\right)^{\beta-\alpha}$.

Having the DOS at hand, the integrals in Eqs. (30) and (31) can be calculated - at least asymptotically - for large $t$. In the classical case Eq. (30) will be dominated by small values of $E$ when $t$ becomes large. The DOS yields

$$
\bar{p}_{\gamma}(t) \sim \begin{cases}t^{-1 / 2} & \text { for } \alpha=1 \\ t^{\alpha / 2-1} & \text { for } \alpha<1\end{cases}
$$

Quantum mechanically one knows that for the NN-case $\bar{\pi}_{\infty}(t) \sim t^{-1}$, see for instance [73]. Considering now the other limiting case, $\gamma=2$, one has [99]

$$
\bar{\pi}_{2}(t)=\left|\int_{0}^{\pi^{2} / 2} d E \frac{\exp (-i E t)}{\pi \sqrt{2} \sqrt{\pi^{2} / 2-E}}\right|^{2} \sim t^{-1} .
$$

Thus, the behavior for long times is the same for $\bar{\pi}_{2}(t)$ and $\bar{\pi}_{\infty}(t)$, which suggests that for all one-dimensional lattices with extensive $(\gamma \geq 2)$ interactions the long time dynamics of the excitations is similar, no matter how long- or short-range the step lengths are. This is in contrast to the classical case, where only CTRW with $\gamma>3$ belong to the same universality class.

These results are corroborated by analytically evaluating $\bar{\pi}_{\gamma}(t)$ using the stationary phase approximation (SPA) [101]. For large $N, \bar{\alpha}_{\gamma}(t)$ can be written in integral form

$$
\bar{\alpha}_{\gamma}(t)=\frac{1}{2 \pi} \int_{0}^{2 \pi} d \theta \exp \left(i E_{\gamma}(\theta) t\right)
$$

The SPA asserts now that the main contribution to this integral comes from those points where $E_{\gamma}(\theta)$ is stationary $\left[d E_{\gamma}(\theta) / d \theta \equiv E_{\gamma}^{\prime}(\theta)=0\right]$. For $\gamma=2, E_{2}(\theta)$ has only one stationary point in $\theta \in\left[0,2 \pi\left[\right.\right.$, namely $\theta_{0}=\pi$, leading to

$$
\bar{\pi}_{2}(t)=\left|\bar{\alpha}_{2}(t)\right|^{2} \approx \frac{1}{2 \pi t\left|E_{2}^{\prime \prime}(\pi)\right|} \sim t^{-1},
$$

which does not show any oscillations and coincides with the long time limit of Eq. (91). For $\gamma>2, E_{\gamma}(\theta)$ has two stationary points in the interval $\theta \in\left[0,2 \pi\left[\right.\right.$, namely $\theta_{0}=0$ and 

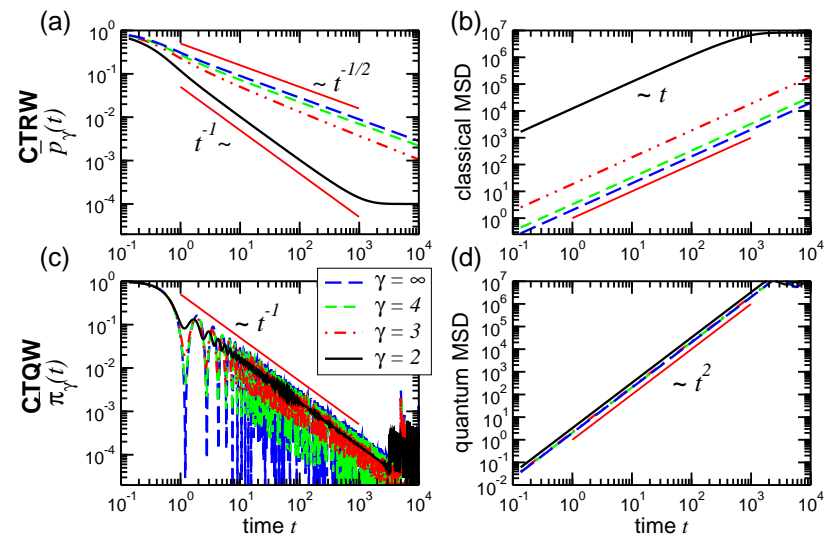

FIG. 27: (Color online) (a) Classical $\bar{p}_{\gamma}(t)$ and (b) quantum mechanical $\bar{\pi}_{\gamma}(t)$ for a discrete ring with $N=10000$ nodes with $\gamma=2,3$, 4 , and $\infty$. From [99].

$\theta_{0}=\pi$. Then $\bar{\alpha}_{\gamma}(t)$ is approximately given by the sum of the contributions of the two stationary points. Consequently [99],

$$
\begin{aligned}
& \bar{\pi}_{\gamma}(t) \approx \frac{1}{2 \pi t}\left(\frac{1}{\left|E_{\gamma}^{\prime \prime}(0)\right|}+\frac{1}{\left|E_{\gamma}^{\prime \prime}(\pi)\right|}\right. \\
& \left.+\frac{2 \cos \left\{t\left[E_{\gamma}(0)-E_{\gamma}(\pi)\right]+\pi / 2\right\}}{\sqrt{\left|E_{\gamma}^{\prime \prime}(0) E_{\gamma}^{\prime \prime}(\pi)\right|}}\right) \sim t^{-1} .
\end{aligned}
$$

The classical and quantum mean square displacements (MSD) are in line with these findings. Now, the MSD for CTRW/CTQW on the discrete ring with initial site $j$ are given by [99]

$$
\left\langle R_{\gamma}^{2}(t)\right\rangle_{\mathrm{cl} ; \mathrm{qm}}=\frac{1}{N} \sum_{k=1}^{N}|k-j|^{2} \mathcal{P}_{k, j}^{(\gamma)}(t),
$$

where $\mathcal{P}_{k, j}^{(\gamma)}(t)=p_{k, j}^{(\gamma)}(t)$ for CTRW and $\mathcal{P}_{k, j}^{(\gamma)}(t)=\pi_{k, j}^{(\gamma)}(t)$ for CTQW. Figure 27 shows numerical calculations of $\bar{p}_{\gamma}(t)$ and $\bar{\pi}_{\gamma}(t)$ for different $\gamma$ and a discrete ring of $N=10000$ nodes. Clearly, $\bar{p}_{\gamma}(t)$ changes when increasing the step width from NN steps to long-range steps, see Fig.27(a). While $\bar{p}_{\gamma}(t)$ for $\gamma>3$ decays as $t^{-1 / 2}$, the power law changes to $t^{-1}$ for $\gamma=2$. In contrast, the decay of the maxima of the quantum return probability $\bar{\pi}_{\gamma}(t)$ follows $t^{-1}$ for all $\gamma$, Fig. 27( $\mathrm{c}$ ). Long-range steps lead only to a damping of the oscillations and to an earlier interference once the excitation has propagated around half of the ring.

As an extension of the ring topology just discussed [99], $\mathrm{Xu}$ considered networks where not only the nearest neighbors are connected but in which each node is connected to its $2 m$ nearest neighbors [102]. This model differs from the one with long-range interactions, where the interaction decreases with the distance $|k-j|$ between the nodes $k$ and $j$.

Now the action of the Hamiltonian on state $|j\rangle$ reads

$$
H|j\rangle=(2 m+1)|j\rangle-\sum_{z=-m}^{m}|j+z\rangle .
$$

It turns out that the Bloch states are also the eigenstates of this Hamiltonian: their eigenvalues read [102]

$$
E_{n}=2 m-2 \sum_{j=1}^{m} \cos \left(j \theta_{n}\right)
$$

Inserting these values into Eqs. (10) and (7) allows to study the dependence of CTQW and of CTRW on $m$. As has been shown by $\mathrm{Xu}$ [102], increasing $m$ results (as intuitively expected) in a faster transport, both for CTQW and CTRW. Morevoer, when considering the long-time average $\chi_{k j}, \mathrm{Xu}$ finds characteristic peaks which depend on $m$. Especially for even $N$, the probability to be at the initial node and the probability to be at the exactly opposite node, i.e., the node $j \pm N / 2$, are not necessary equal [102], as it is the case for only nearestneighbor couplings. Depending on $m$, these two values may differ [102]: an explanation of this effect is still lacking.

\section{B. Systems with disorder and localization}

In real physical systems, under the influence of the surroundings, the couplings between the nodes may differ. In a static picture, one can introduce disorder by adding to the unperturbed Hamiltonian $\boldsymbol{H}_{0}$ a disorder operator $\boldsymbol{\Delta}$, i.e., by setting $\boldsymbol{H}=\boldsymbol{H}_{0}+\boldsymbol{\Delta}$ [103]. The disorder matrix $\boldsymbol{\Delta}=\left(\Delta_{l, j}\right)$ is taken to have non-zero entries only at the positions for which $H_{l, j} \neq 0$. For different strengths of disorder, the elements $\Delta_{l, j}=\Delta_{j, l}$ are chosen randomly (drawn from a normal distribution with the zero mean and unit variance, and then multiplied by a factor of $\Delta$ which takes values from the interval $[0,1 / 2])$. Note that under these assumptions for the (static) disorder the connectivity of the graph is essentially unchanged, i.e., there are no new connections created nor are existing connections destroyed. Therefore, the only non-zero matrix elements of $\boldsymbol{H}$ are those of the initial $\boldsymbol{A}$. The action of the new Hamiltonian $\boldsymbol{H}$ on a state $|j\rangle$ reads then

$$
\begin{aligned}
& \boldsymbol{H}|j\rangle=\left(\boldsymbol{H}_{0}+\boldsymbol{\Delta}\right)|j\rangle \\
& =2|j\rangle-|j-1\rangle-|j+1\rangle \\
& +2 \Delta_{j, j}|j\rangle-\Delta_{j, j-1}|j-1\rangle-\Delta_{j, j+1}|j+1\rangle .
\end{aligned}
$$

In the following two cases of disorder are considered:

(A) Diagonal disorder (DD), where $\Delta_{j, j} \neq 0$ and $\Delta_{l, j}=0$ for $l \neq j$. Here, a random number is assigned to each $\Delta_{j, j}$, a procedure which leads to $N$ random numbers.

(B) Diagonal and off-diagonal disorder (DOD), where a random number is chosen for each $\Delta_{j, j}$ and for each $\Delta_{j, j-1}$. For this, $2 N$ random numbers are needed.

Introducing disorder into the system in this way has consequences for the relation between the CTQW and the CTRW. In CTRW the transition rates, given by the entries of the the transfer matrix $\boldsymbol{T}$, are correlated, i.e., for each site the sum of the non-diagonal rates for transmission from it and the diagonal rate of leaving it are the same. In the cases considered here, a direct identification of the Hamiltonian $\boldsymbol{H}$ with a classical transfer matrix is not possible anymore. However, the 
(a) $\mathrm{N}=100$

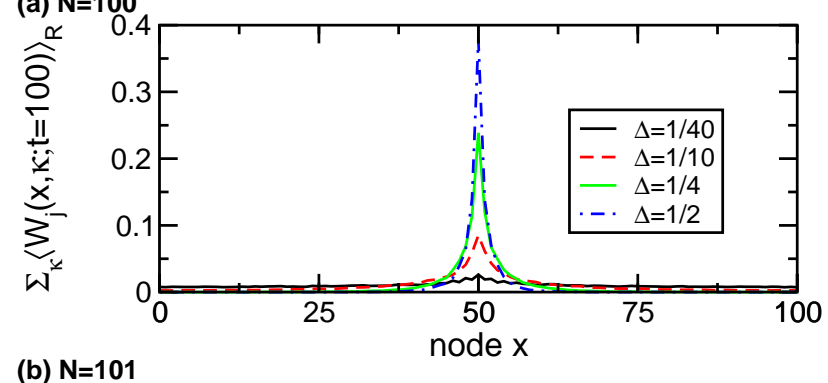

(b) $\mathrm{N}=101$

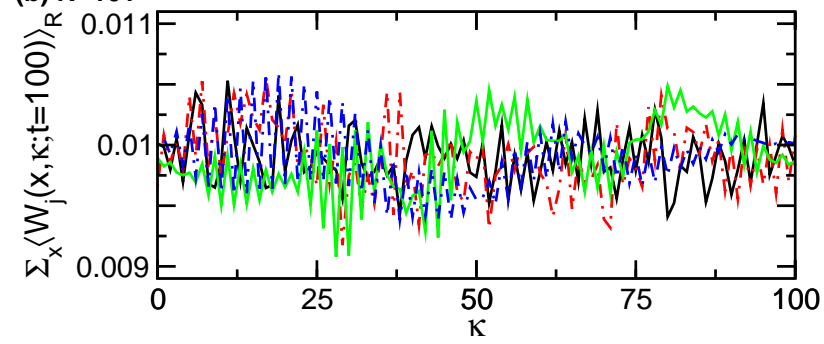

FIG. 28: (Color online) Long-time average $\chi_{k, j}$ for different $\Delta$ and (a) $N=100$ and (b) $N=101$. From [103].

DOD and DD Hamiltonians are widely used in quantum mechanical nearest-neighbor hopping models, to which also the CTQW belong. Furthermore, we still consider transport processes on graphs which have the connectivity matrix $\boldsymbol{A}$, but the direct connection between $\boldsymbol{H}$ and $\boldsymbol{T}$ is lost.

Now, consider again rings of $N$ nodes, where at time $t=$ 0 the excitation is assumed to be localized at node $j$. The above system is similar to the Anderson model [11], which has been found to show (strong) localization around the initial condition. The same happens here. In the ensemble average as well as for single realizations, an excitation starting at $j$ remains localized in the vicinity of $j$ [103]. This effect is best seen in the long-time average. Figure 28 shows $\chi_{k, j}$ for two different ring sizes, $N=100$ and $N=101$, and varying $\Delta$. Clearly, the larger is $\Delta$ the more is the long time average localized around the initial node $j=50$.

Quantum walks in random linear environments were also studied by Yin et al. [104]. They calculated numerically the quantum carpet structures for DD. It turns out that in the course of time the excitation stays localized around its initial node; also depending on the strength of the disorder, some structure of the original quantum carpets may still remain visible.

Yin et al. also studied dynamic diagonal disorder, where the diagonal elements of $\boldsymbol{H}$ are rapidly varying with time and the update of $(H)_{j j}$ is done at times comparable or much smaller than the time step of the numerically determined dynamical changes [104]. For dynamic disorder, the interference patterns making up the quantum carpets are washed out. However, distinct from the case of static disorder, no localization can be seen. Moreover, after the temporal range in which interference is lost, the dynamics becomes classical. This crossover behavior is also observed in the mean square displacement, which changes in a certain time range - depending on the strength of disorder - from the ballistic behavior $\sim t^{2}$ to the diffusive behavior $\sim t[104]$.

\section{SYSTEMS WITH ABSORPTION}

In general, an excitation does not stay forever in the system in which it was created; the excitation either decays (radiatively or by exciton recombination) or, e.g., in the case of biological light-harvesting systems, it gets absorbed at the reaction center, where it is transformed into chemical energy. In such cases, the total probability to find the excitation within the network is not conserved. Such loss processes can be modelled phenomenologically by changing the transfer matrix or the Hamiltonian [50, 105- 107]. To fix the ideas, we consider networks in which the excitation can only vanish at certain nodes. These nodes will be called trap-nodes or traps. In the absence of traps, let the transfer matrix and the Hamiltonian of the corresponding network be $\boldsymbol{T}_{0}$ and $\boldsymbol{H}_{0}$, respectively. Take now $M$ out to the $N$ total nodes to be traps and denote them by $m$, so that $m \in \mathcal{M}$, with $\mathcal{M} \subset\{1, \ldots, N\}$. The trapping process is now modelled by introducing a trapping matrix $\Gamma$ which is given by a sum over all trap nodes; $\boldsymbol{\Gamma}$ has only diagonal elements, i.e.,

$$
\boldsymbol{\Gamma} \equiv \sum_{m} \Gamma_{m}|m\rangle\langle m|
$$

(in the following one assumes that $\Gamma_{m}=\Gamma>0$ for all $m$ ).

CTRW with decreasing exciton probabilities due to trapping are well described through the following transfer matrix:

$$
T \equiv T_{0}-\Gamma .
$$

The total Hamiltonian $\boldsymbol{H}$ corresponding to trapping is then:

$$
\boldsymbol{H} \equiv \boldsymbol{H}_{0}-i \boldsymbol{\Gamma} .
$$

Note that the connection between CTRW and CTQW is now less direct than before. For CTRW the term corresponding to trapping has only real elements and the total transfer matrix stays real. For CTQW, however, the trapping term has purely imaginary elements. As a result, $\boldsymbol{H}$ is non-hermitian and has $N$ complex eigenvalues, $E_{l}=\epsilon_{l}-i \gamma_{l}(l=1, \ldots, N)$. In general, $\boldsymbol{H}$ has $N$ left and $N$ right eigenstates $\left|\Phi_{l}\right\rangle$ and $\left\langle\tilde{\Phi}_{l}\right|$, respectively. It turns out that in most cases the eigenstates of $\boldsymbol{H}$ form a complete and biorthonormal set, see, e.g., Ref. [108],

$$
\sum_{l=1}^{N}\left|\Phi_{l}\right\rangle\left\langle\tilde{\Phi}_{l}\right|=\mathbf{1} \quad \text { and } \quad\left\langle\tilde{\Phi}_{l} \mid \Phi_{l^{\prime}}\right\rangle=\delta_{l l^{\prime}}
$$

Both, eigenvalues and eigenstates, will be different for CTRW and $\mathrm{CTQW}$, because the incorporation of the trapping process is different.

If the trapping strength $\Gamma$ is small compared to the couplings between neighboring nodes, perturbation theory allows to relate the real part of the eigenvalues to the eigenvalues of the unperturbed Hamiltonian $\boldsymbol{H}_{0}$. Let $\left|\Psi_{l}^{(0)}\right\rangle$ be the $l$ th eigenstate and $E_{l}^{(0)} \in \mathbb{R}$ be the $l$ th eigenvalue of the unperturbed 
system with Hamiltonian $\boldsymbol{H}_{0}$. Up to first-order, the eigenvalues of the perturbed system are given by [105]

$$
E_{l}=E_{l}^{(0)}+E_{l}^{(1)}=E_{l}^{(0)}-i \Gamma \sum_{m \in \mathcal{M}}\left|\left\langle m \mid \Psi_{l}^{(0)}\right\rangle\right|^{2} .
$$

Therefore, the correction term determines the imaginary parts $\gamma_{l}$, while the unperturbed eigenvalues are the real parts $\epsilon_{l}=$ $E_{l}^{(0)}$. Moreover, the imaginary parts are solely determined by the contribution of the eigenstates of the network without traps at the trap nodes $m$.

\section{A. Average survival probability}

In an ideal experiment one would excite exactly one node, say $j \notin \mathcal{M}$, and read out the outcome $\pi_{k j}(t)$, i.e., the probability to be at node $k \notin \mathcal{M}$ at time $t$. However, it is easier to keep track of the total outcome at all nodes $k \notin \mathcal{M}$, namely of $\sum_{k \notin \mathcal{M}} \pi_{k j}(t)$. Since the states $|k\rangle$ form a complete, orthonormal basis set one has $\sum_{k \notin \mathcal{M}}|k\rangle\left\langle k\left|=\mathbf{1}-\sum_{m \in \mathcal{M}}\right| m\right\rangle\langle m|$, which leads to [50]:

$$
\begin{aligned}
& \sum_{k \notin \mathcal{M}} \pi_{k j}(t)=\sum_{k \notin \mathcal{M}}\left|\alpha_{k j}(t)\right|^{2} \\
& =\sum_{l=1}^{N} e^{-2 \gamma_{l} t}\left\langle j \mid \Phi_{l}\right\rangle\left\langle\tilde{\Phi}_{l} \mid j\right\rangle-\sum_{l, l^{\prime}=1}^{N} e^{-i\left(E_{l}-E_{l^{\prime}}^{*}\right) t} \\
& \times \sum_{m \in \mathcal{M}}\left\langle j \mid \Phi_{l^{\prime}}\right\rangle\left\langle\tilde{\Phi}_{l^{\prime}} \mid m\right\rangle\left\langle m \mid \Phi_{l}\right\rangle\left\langle\tilde{\Phi}_{l} \mid j\right\rangle .
\end{aligned}
$$

By averaging over all $j \notin \mathcal{M}$, the mean survival probability is given by [50]

$$
\begin{aligned}
& \Pi_{M}(t) \equiv \frac{1}{N-M} \sum_{j \notin \mathcal{M}} \sum_{k \notin \mathcal{M}} \pi_{k j}(t) \\
& =\frac{1}{N-M}\left\{\sum_{l=1}^{N} e^{-2 \gamma_{l} t}\left[1-2 \sum_{m \in \mathcal{M}}\left\langle\tilde{\Phi}_{l} \mid m\right\rangle\left\langle m \mid \Phi_{l}\right\rangle\right]\right. \\
& \left.+\sum_{l, l^{\prime}=1}^{N} e^{-i\left(E_{l}-E_{l^{\prime}}^{*}\right) t}\left[\sum_{m \in \mathcal{M}}\left\langle\tilde{\Phi}_{l^{\prime}} \mid m\right\rangle\left\langle m \mid \Phi_{l}\right\rangle\right]^{2}\right\}
\end{aligned}
$$

For long $t$ and small $M / N$, Eq. (106) simplifies considerably: At long times the oscillating term on the right hand side drops out and for small $M / N$ one has $2 \sum_{m \in \mathcal{M}}\left\langle\tilde{\Phi}_{l} \mid m\right\rangle\left\langle m \mid \Phi_{l}\right\rangle \ll 1$. Thus, $\Pi_{M}(t)$ is mainly a sum of exponentially decaying terms [50]:

$$
\Pi_{M}(t) \approx \frac{1}{N-M} \sum_{l=1}^{N} \exp \left[-2 \gamma_{l} t\right]
$$

Asymptotically, Eq. 107) is dominated by the $\gamma_{l}$ values closest to zero. If the smallest one, $\gamma_{\min }$, is well separated from the other values, one is led for $t \gg 1 / \gamma_{\min }$ to the exponential decay found in earlier works, $\Pi_{M}(t)=\exp \left(-2 \gamma_{\min } t\right)$ [109].
Such long times are not of much experimental relevance (see also below), since most measurements highlight shorter times, at which many $\gamma_{l}$ contribute. In the corresponding energy range the $\gamma_{l}$ often scale, so that in a large $l$ range one finds $\gamma_{l} \sim a l^{\mu}$. The prefactor $a$ depends only on $\Gamma$ and $N$ [109]. For densely distributed $\gamma_{l}$ and at intermediate times one has, from Eq. [107, [50]

$$
\begin{aligned}
\Pi_{M}(t) & \approx \int d x e^{-2 a t x^{\mu}} \\
& =\int d y \frac{e^{-y^{\mu}}}{(2 a t)^{-1 / \mu}} \sim t^{-1 / \mu} .
\end{aligned}
$$

Analogously, the mean survival probability for CTRW is given by

$$
P_{M}(t) \equiv \frac{1}{N-M} \sum_{j \notin \mathcal{M}} \sum_{k \notin \mathcal{M}} p_{k j}(t) .
$$

If the smallest eigenvalue, $\lambda_{1}$, is well separated from the rest, $P_{M}(t)$ turns very quickly into a simple exponential decay. Then, for not too small times, it can be shown that [105]

$$
P_{M}(t) \approx \frac{1}{N-M} e^{-\lambda_{1} t}\left|\sum_{k \notin \mathcal{M}}\left\langle k \mid q_{1}\right\rangle\right|^{2} .
$$

\section{B. Regular networks}

\section{Ring with traps}

Some of the nodes of a ring of $N$ nodes are now taken to be traps. Depending on the particular choice of trap arrangements, the average survival probability $\Pi_{M}(t)$ shows different features [106].

As already discussed, the eigenstates of the ring without traps are Bloch states. Furthermore, if the trapping strength is small compared to the interaction strength between the nodes, the imaginary parts of the Hamiltonian of a network with traps are given by the contributions of the eigenstates at the trap positions, see Eq. (103). However, since all eigenvalues except $E_{1}=0$ (and for even $N$ also except $E_{N / 2}=4$ ) are two-fold degenerate, some care is in order when applying perturbation theory.

Take now $N$ to be even. Then, for $l=1$ and for $l=N / 2$ one has

$$
E_{l}^{(1)}=-i \Gamma \sum_{m \in \mathcal{M}}\left|\left\langle m \mid \Phi_{l}^{(0)}\right\rangle\right|^{2}
$$

Furthermore,

$$
E_{1}=4-i \Gamma \frac{M}{N} \quad \text { and } \quad E_{N / 2}=-i \Gamma \frac{M}{N} .
$$

For $l$ different from 1 and from $N / 2$ one sets

$$
V_{i, j} \equiv\left\langle\Phi_{i}^{(0)}|-i \boldsymbol{\Gamma}| \Phi_{j}^{(0)}\right\rangle
$$


and applies the expression valid for two-fold degenerate solutions of $\boldsymbol{H}_{0}$ [106]:

$$
\begin{aligned}
& E_{l}^{(1)}=\frac{1}{2}\left(V_{l, l}+V_{N-l, N-l}\right) \\
& \pm \frac{1}{2}\left[\left(V_{l, l}-V_{N-l, N-l}\right)^{2}+4\left|V_{l, N-l}\right|^{2}\right]^{1 / 2}
\end{aligned}
$$

where one takes the positive sign for $l \in[1, N / 2-1]$ and the negative sign for $l \in[N / 2+1, N-1]$. Now one has

$$
V_{l, l} \equiv V_{N-l, N-l}=-i \Gamma \frac{M}{N},
$$

independently of the trap arrangement and

$$
\begin{aligned}
& V_{l, N-l}=-i \frac{\Gamma}{N} \sum_{j=1}^{M} \exp \left\{2 i \pi m_{j}[l-(N-l)] / N\right\} \\
& =-i \frac{\Gamma}{N} \sum_{j=1}^{M} \exp \left(4 i \pi l m_{j} / N\right) .
\end{aligned}
$$

Inserting the last results into Eq. (114) yields [106]

$$
E_{l}^{(1)}=\frac{-i \Gamma}{N}\left(M \pm\left|\sum_{j=1}^{M} e^{2 i \pi 2 l m_{j} / N}\right|\right) .
$$

Notice that for special trap arrangements the $E_{l}^{(1)}$ can be calculated exactly: The most striking results are obtained when the exponential in the sum in Eq. (117) equals one of the values from the set $\{1, i,-1,-i\}$. Then the absolute value of the sum reduces to $\left|\sum_{j=1}^{M} \exp \left(i 4 \pi l m_{j} / N\right)\right|=M$.

Now, for a single trap, which without loss of generality is placed at position $m_{j}=j=1$, one has $E_{l}^{(1)}=-i \Gamma / N[1 \pm$ $\exp (i 4 \pi l / N)]$. Therefore, one has $E_{l}^{(1)}=0$ for $l=N$ and for $l=N / 2$. As a consequence, $\Pi_{M}(t)$ will not decay to zero but to a constant value given by $1 /(N-1)$.

Another example is a periodic distribution of traps with $m_{j}=j N / M$, while $N / M \in \mathbb{N}$, see Fig. 29(a). Then it is straightforward to show that if $2 l / M \in \mathbb{N}$ the sum in Eq. (117) equals one of the values from the set $\{1, i,-1,-i\}$. The total number of such values is given by [106]

$$
|\Upsilon|= \begin{cases}\lfloor(N-2) / M\rfloor & \text { for even } M, \\ \lfloor(N-2) / 2 M\rfloor & \text { for odd } M,\end{cases}
$$

where $\lfloor x\rfloor$ denotes the largest integer less than or equal to $x$. In particular, for both $M=1$ and $M=2,|\Upsilon|=N / 2-1$. Hence, for large structures with $M \ll N, \Pi_{M}(t)$ decays asymptotically to $1 / M$ (even case) and to $1 /(2 M)$ (odd case). Figure 30 shows results obtained for a ring of size $N=300$ with a periodic arrangement of $M=10(|\Upsilon|=29)$ and $M=75(|\Upsilon|=1)$ traps. Consequently, the survival probability $\Pi_{M}(t)$ decays to the constant values $1 / 10$ and $1 / 225$, respectively. From a physical point of view, the finite limit for the survival probability stems from the existence of stationary states to which the nodes in $\mathcal{M}$ do not contribute, so that (a) periodic arrangement of traps

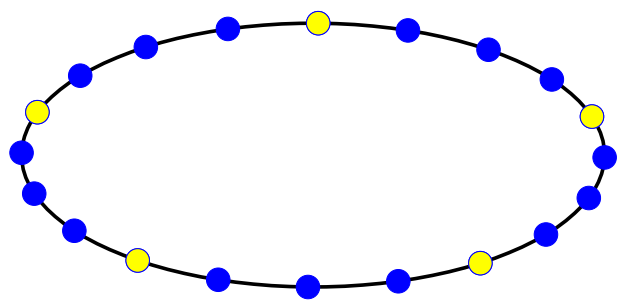

(b) sequential arrangement of traps

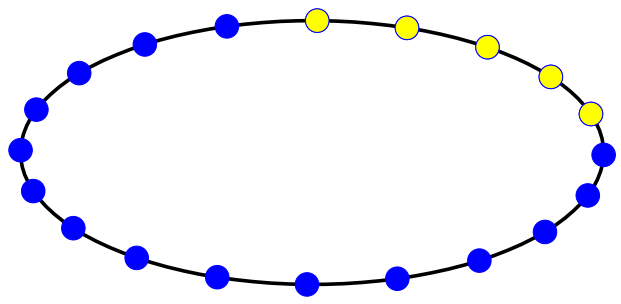

FIG. 29: Periodic (a) and sequential (b) arrangements of traps on a ring.

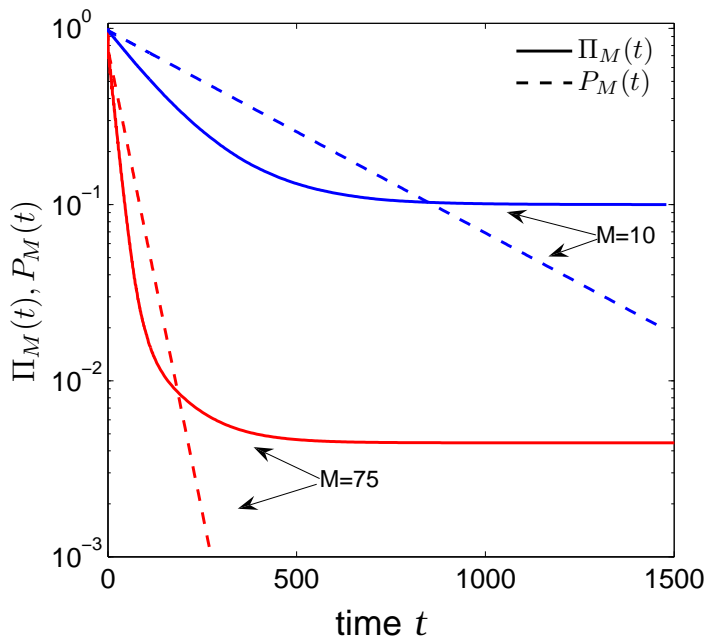

FIG. 30: Survival probabilities $\Pi_{M}(t)$ (continuous line) and $P_{M}(t)$ (dotted lines) on a ring of size $N=300$ and $\Gamma=0.01$ in the presence of $M=10$ and of $M=75$ traps arranged periodically, i.e. $m_{j}=j N / M$. Note the semilogarithmic scales. From [106].

they never "see" the traps. This genuine quantum-mechanical effect has no counterpart in the classical world where, for finite structures, the survival probability always decays to zero in the presence of traps. In particular, as shown in Fig. 30 , $P_{M}(t)$ decays exponentially, as expected.

For a sequential arrangement of traps, such that $m_{j}=j$ 


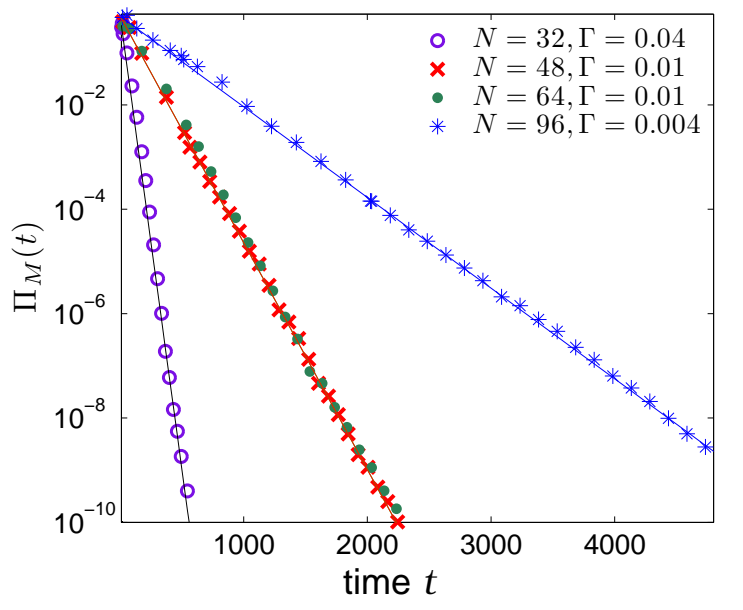

FIG. 31: Survival probability $\Pi_{M}(t)$ on rings of size $N=32,48,64$ and 96 with a sequential arrangement of $M=N / 2$ traps for $\Gamma=$ $0.04,0.01,0.004$, as indicated.The straight lines represent Eq. 121. From [106].

and $j=1, \ldots ., M$, see Fig. 29 b), Eq. 116 can be written as

$$
\begin{aligned}
V_{l, N-l}=-i \Gamma / N \sum_{j=1}^{M} \exp (4 i \pi l j / N) \\
=\frac{-i \Gamma}{N} \frac{\exp (4 i \pi l M / N)-1}{\exp (4 \pi i l / N)-1} \exp (4 \pi i l / N) \\
=\frac{-i \Gamma}{N} \frac{\sin (2 \pi M l / N)}{\sin (2 \pi l / N)} \exp [2 i \pi l(M+1) / N],
\end{aligned}
$$

which yields

$$
E_{l}^{(1)}=\frac{-i \Gamma}{N}\left(M \pm \frac{\sin (2 \pi M l / N)}{\sin (2 \pi l / N)}\right) .
$$

Notice that since $l \neq N / 2$ and $l \neq N$ then $2 l / N \notin \mathbb{N}$, while for $2 l M / N \in \mathbb{N}$ then $E_{l}^{(1)}=E_{N-l}^{(1)}=-i \Gamma M / N$. In particular, when $M=N / 2, \gamma_{l}=M / N$ for each value of $l \in[1, N]$. As a result, and by neglecting oscillations, one has [106]

$$
\Pi_{M}(t) \approx \frac{M}{N-M} e^{-2 \Gamma t M / N} \sim e^{-\Gamma t},
$$

which is independent of $N$. As shown in Fig. 31, the exponential behaviour predicted by Eq. 121) holds also for intermediate times.

\section{Line with traps}

An example of a network with traps which allows to study different time scales is a finite line of nodes with traps at each end. The Hamiltonian is thus [50]

$$
\begin{aligned}
\boldsymbol{H}= & \sum_{n=2}^{N-1}(2|n\rangle\langle n|-| n-1\rangle\langle n|-| n+1\rangle\langle n|) \\
& +|1\rangle\langle 1|-| 2\rangle\langle 1|+| N\rangle\langle N|-| N-1\rangle\langle N| \\
& +i \Gamma(|1\rangle\langle 1|+| N\rangle\langle N|) .
\end{aligned}
$$

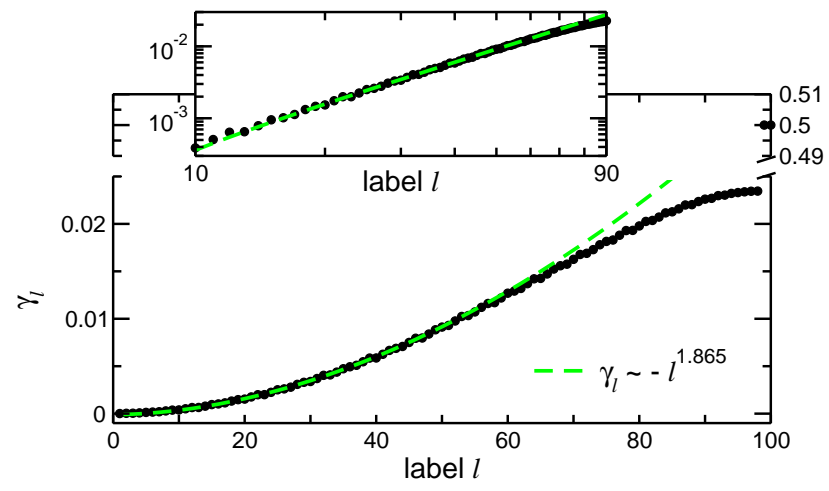

FIG. 32: (Color online) Imaginary parts $\gamma_{l}$ (dots) in ascending order for $N=100$ and $\Gamma=1$. Note the shortened $y$ axis. The inset shows $\gamma_{l}$ in log-log scale for $l=10, \ldots, 90$. From [50].

Without loss of generality, an eigenstate of the finite chain without traps can be written as $(l=1, \ldots, N)$ [105]

$$
\left|\Psi_{l}^{(0)}\right\rangle= \begin{cases}\sqrt{\frac{1}{N}} \sum_{j=1}^{N}|j\rangle & \text { for } l=N \\ \sqrt{\frac{2}{N}} \sum_{j=1}^{N} \cos \left[(2 j-1) \theta_{l} / 2\right]|j\rangle & \text { else, }\end{cases}
$$

where for convenience one takes $\theta_{l} \equiv \pi(N-l) / N \in[0, \pi[$; the corresponding eigenvalues are $E_{l}^{(0)}=2-2 \cos \theta_{l}$ (note that the smallest eigenvalue is $E_{N}^{(0)}=0$ ). Thus, first order perturbation theory yields from Eqs. (103) and (123) as imaginary parts $\gamma_{N}=2 \Gamma / N$ and $\gamma_{l}=(4 \Gamma / N) \cos ^{2}\left(\theta_{l} / 2\right)$ for $l=1, \ldots, N-1$. Indeed, for $l \ll N$ this means that $\gamma_{l} \sim l^{2}$, so that the average survival probability scales in the corresponding time interval as $\Pi_{M}(t) \sim t^{-1 / 2}$, see Eq. (108).

If the trapping stength increases, one cannot employ perturbation theory anymore. Nevertheless, one can always calculate the eigenvalues of $\boldsymbol{H}$ numerically. As it will turn out, the scaling $\gamma_{l} \sim l^{\mu}$ still holds in this case and extends even over a wider range of $l$-values. Figure 32 shows the spectrum of $\gamma_{l}$ for $N=100$ and $\Gamma=1$; the double logarithmic plot (see inset) demonstrates that scaling holds for $10 \leq l \leq 60$, where the exponent $\mu$ is about $\mu=1.865$.

Figure 33 compares, for a linear system with $N=100$ and $\Gamma=1$, the classical $P_{M}(t)$ to the quantum mechanical survival probability $\Pi_{M}(t)[50]$. Evidently, $P_{M}(t)$ and $\Pi_{M}(t)$ differ strongly: the $P_{M}(t)$ decay established for CTRW is practically exponential. $\Pi_{M}(t)$, on the other hand, shows two regimes: a power-law decay at intermediate times (panel (a)) and an exponential decay (panel (b)) at very long times.

Turning now to the parameter dependences of $\Pi_{M}(t)$, Fig. 34]shows the dependence of $\Pi_{M}(t)$ on $N$ [50]. Note that the scaling regime, where $\Pi_{M}(t) \sim t^{-1 / \mu}$ holds, gets larger with increasing $N$. The cross-over to this scaling region from the domain of short times occurs around $t \approx N / 2$. For larger $N$ and in the intermediate time domain, $\Pi_{M}(t)$ scales nicely with $N$. In this case, the power-law approximation [Eq. (108)] holds and by rescaling $l$ to $l / N$ one has from Eq. (107) that 


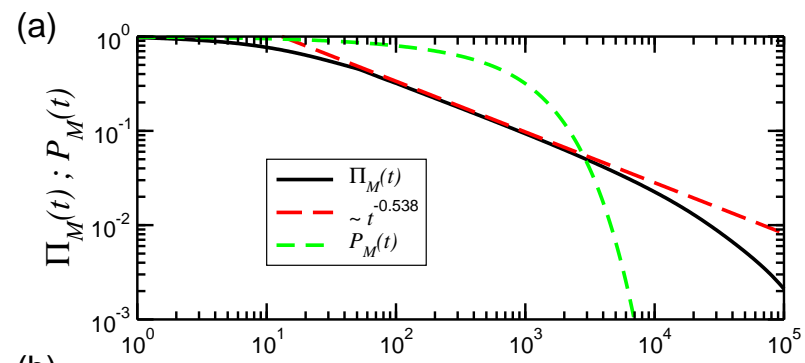

(b)

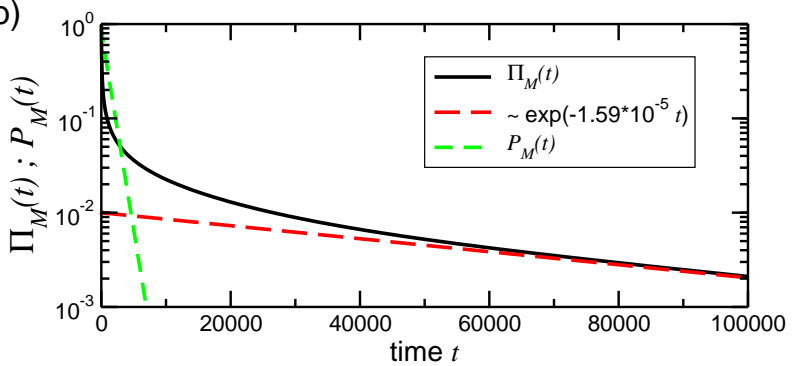

FIG. 33: (Color online) Temporal decay of $\Pi_{M}(t)$ (solid black lines) and $P_{M}(t)$ (short dashed green lines) for $N=100$ and $\Gamma=1$ in (a) double logarithmic scales and in (b) logarithmic scales. Indicated are the fits to $\Pi_{M}(t)$ (long dashed lines) in the intermediate (upper red) and the long (lower blue) time regime. From [50].

[50]

$$
\begin{aligned}
\Pi_{M}(t) & \sim \sum_{l} e^{-2 N^{-3} l^{\mu} t} \\
& =\sum_{l} \exp \left[-2(l / N)^{\mu} N^{-(3-\mu)} t\right]
\end{aligned}
$$

where it was assumed that $a \sim N^{-3}$ for a linear system [109]. Thus, when rescaling $l$ to $l / N$, the time has to be rescaled by the factor $N^{-(3-\mu)}$. Indeed, all curves for which a powerlaw behavior is visible fall on a master curve; see the inset in Fig. 34

\section{Line with traps and long-range interactions}

As mentioned above, the interaction range does not need to be restricted to nearest neighbor interactions (NNI). When the interactions between two nodes go as $R^{-\nu}$, the Hamiltonian of the networks without traps has the following structure [105]:

$$
\begin{aligned}
& \boldsymbol{H}_{0}(\nu)=\sum_{n=1}^{N}\left[\sum_{R=1}^{n-1} R^{-\nu}(|n\rangle\langle n|-| n-R\rangle\langle n|)\right. \\
& \left.+\sum_{R=1}^{N-n} R^{-\nu}(|n\rangle\langle n|-| n+R\rangle\langle n|)\right]
\end{aligned}
$$

Note that in the case of a line, the states $|k\rangle$ with $k \notin$ $\{1, \ldots, N\}$ are implicitly excluded from the summation.

For large exponents $\nu$ the LRI can be regarded as a small perturbation to the NNI, i.e., having $\boldsymbol{H}_{0}(\nu)=\boldsymbol{H}_{0}+\boldsymbol{H}_{\nu}$,
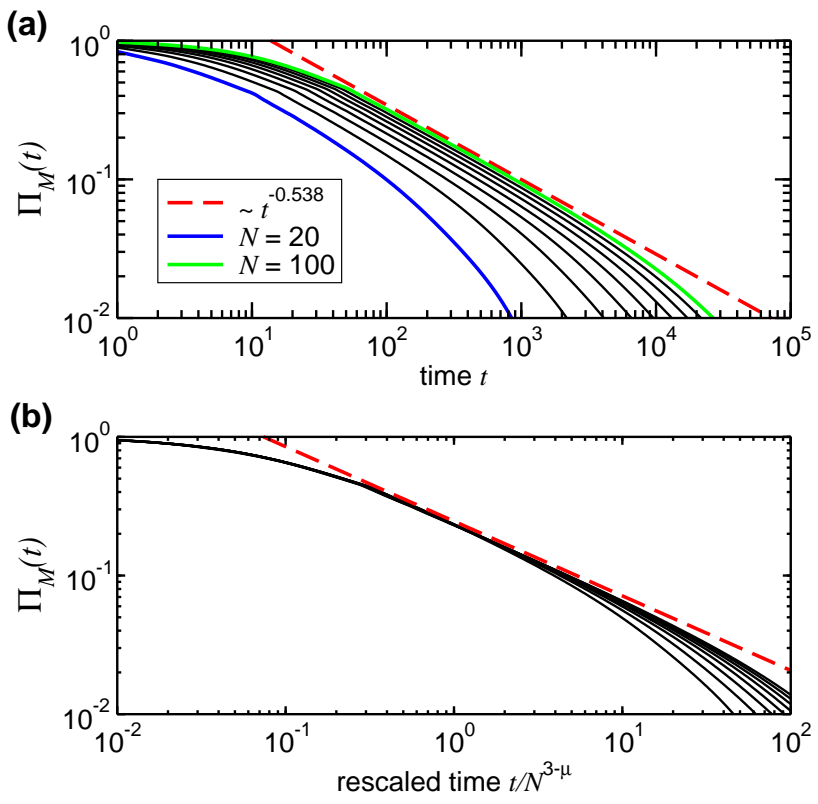

FIG. 34: (Color online) Panel (a) shows the $N$-dependence of $\Pi_{M}(t)$ for $\Gamma=1 ; N$ increases in steps of 10 from 20 (blue line) to 100 (green line). Panel (b) shows $\Pi_{M}(t)$ versus the rescaled time $t / N^{3-\mu}$. From [50].

where $\boldsymbol{H}_{\nu}$ contains only the correction terms to the NNI case $\boldsymbol{H}_{0}$. This allows one to calculate from the unperturbed states $\left|\Psi_{l}^{(0)}\right\rangle$ the perturbed eigenstates $\left|\Psi_{l}\right\rangle$ up to first order. Taking the states $\left|\Psi_{l}\right\rangle$ to be the eigenstates of the LRI system without traps, one readily obtains the imaginary parts $\gamma_{l}$ for small trapping strength from Eq. (103) as $\gamma_{l}=2 \Gamma\left|\left\langle 1 \mid \Psi_{l}\right\rangle\right|^{2}$, where [105]

$$
\left\langle 1 \mid \Psi_{l}\right\rangle=\left\langle 1 \mid \Psi_{l}^{(0)}\right\rangle+\sum_{r \neq l} \frac{\left\langle\Psi_{r}^{(0)}\left|\boldsymbol{H}_{\nu}\right| \Psi_{l}^{(0)}\right\rangle}{E_{l}^{(0)}-E_{r}^{(0)}}\left\langle 1 \mid \Psi_{r}^{(0)}\right\rangle .
$$

For large $\nu$ the coupling to the next-next-nearest neighbor is by a factor of $(3 / 2)^{\nu}$ smaller, for $\nu=10$ this is about one and a half orders of magnitude. Taking, for fixed $\nu$, only nearest and next-nearest neighbor couplings into account allows one to obtain simple analytic expressions. Thus, Eq. (126) yields

$$
\begin{aligned}
\left\langle 1 \mid \Psi_{l}\right\rangle= & \sqrt{\frac{2}{N}} \cos \left(\frac{\theta_{l}}{2}\right) \\
& +2^{-\nu} \sqrt{\frac{2}{N}} \sin \left(2 \theta_{l}\right) \sin \left(\frac{\theta_{l}}{2}\right),
\end{aligned}
$$

$\left(\theta_{l} \equiv \pi(N-l) / N \in[0, \pi[)\right.$ which results in [105]

$$
\gamma_{l} \approx \gamma_{l}^{(0)}+2^{-\nu} \gamma_{l}^{(1)}+\mathcal{O}\left(2^{-2 \nu}\right),
$$

where $\gamma_{l}^{(0)}$ is the NNI expression, discussed in Sec. VIB 2 and $\gamma_{l}^{(1)}=(8 \Gamma / N) \cos \left(\theta_{l} / 2\right) \sin \left(2 \theta_{l}\right) \sin \left(\theta_{l} / 2\right)$ is the correction due to the LRI. The smallest $\gamma_{l}$-values are those for which $l \ll N$, which leads to a decrease of the imaginary 

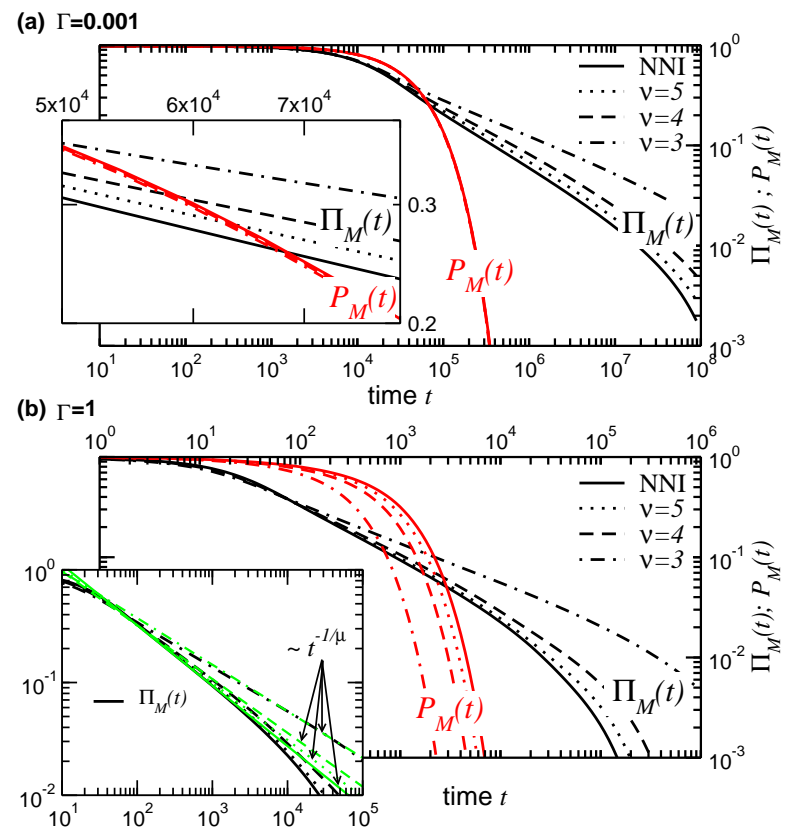

FIG. 35: (Color online) $\nu$-dependence of the quantum mechanical $\Pi_{M}(t)$ and the classical $P_{M}(t)$ decay behaviors for a chain of $N=$ 100 sites; here (a) $\Gamma=0.001$ and (b) $\Gamma=1$. The inset in (a) shows a close-up picture of the region where $\Pi_{M}(t)$ and $P_{M}(t)$ cross. The inset in (b) shows power-law fits to $\Pi_{M}(t)$ in the intermediate time regime with exponents $1 / \mu$, where the $\mu$ are taken from Fig. 3(b) of Ref. [105]. From [105].

parts $\gamma_{l}$ because $\gamma_{l}^{(1)}<0$ for $l \ll N$. Here, one can approximate the imaginary parts by a power-law, i.e., $\gamma_{l} \sim l^{\mu}$. A rough estimate of the scaling exponent $\mu$, assuming $\nu \gg 1$, can be readily given [105]:

$$
\mu \approx \frac{\ln \gamma_{l+1}-\ln \gamma_{l}}{\ln (l+1)-\ln l} \approx \mu^{(0)}+2^{-\nu} \mu^{(1)}
$$

Since $\mu^{(1)}$ is strictly positive for small $l$, the inclusion of LRI leads to a decrease of $\gamma_{l}$ when compared to the NNI case. In turn, this results in a slower decay of $\Pi_{M}(t)$.

Figure 35 displays for comparison the quantum mechanical $\Pi_{M}(t)$ and the classical $P_{M}(t)$ behaviors for different $\nu$ and $\Gamma ; \Pi_{M}(t)$ and $P_{M}(t)$ were obtained by numerically diagonalizing the corresponding Hamiltonian $\boldsymbol{H}(\nu)$ and transfer matrix $\boldsymbol{T}(\nu)$, respectively [105]. Clearly, for both $\Gamma$-values the LRI lead to a slower decay of $\Pi_{M}(t)$, i.e., to a slower trapping of the excitation, which is somewhat counterintuitive since the opposite effect is observable for classical systems, where the decay of $P_{M}(t)$ becomes faster for decreasing $\nu$, see below. By increasing the trapping strength $\Gamma$, the difference between the quantum and the classical behaviors become even more pronounced, compare Figs. 35 (a) and 35(b). Generally, for $\Pi_{M}(t)$ a change in $\Gamma$ leads mainly to in a rescaled time axis, since the imaginary parts $\gamma_{l}$ turn out to be of the same order of magnitude when rescaled by $\Gamma$.

$\mathrm{Xu}$ has studied a ring where the interaction strength does not decay with distance, as in [105], but ranges from a given node to the $m$ nearest neighbors [110]. By choosing for

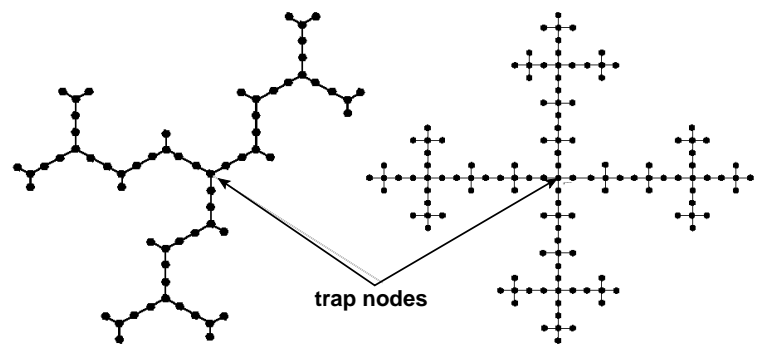

FIG. 36: Examples of two regular hyperbranched fractals of generation $g=3$ with functionalities $f=3$ (left) and $f=4$ (right).

$N=100$ five nodes randomly as trap nodes, $\mathrm{Xu}$ has shown numerically that both the CTQW and the CTRW survival probabilities decay with increasing $m$ [110], thus showing an opposite effect to that mentioned above. However, an explanation was not given.

\section{Fractals}

\section{Hyperbranched fractals}

Unlike the regular network discussed in the previous sections, hyperbranched fractals allow one to study the survival probability in the presence of highly degenerate eigenvalues. Volta investigated so-called regular hyperbranched fractals, see Fig. 36, for which the eigenvalue spectra of the connectivity matrices can be calculated recursively [111]. As shown previously [112], the eigenvalues of the $(g+1)$ st generation can be obtained from the eigenvalues of the $g$ th generation by solving

$$
P\left(\lambda^{g+1}\right)=\lambda^{g},
$$

where $P(\lambda)=\lambda(\lambda-3)(\lambda-f-1), f$ being the functionality of the fractal. The three roots of the polynomial are given by the Cardano solutions [112]

$$
\lambda_{j}=\frac{f+4}{7}+\frac{2}{3}|f(f-1)+7|^{1 / 2} \cos [(\phi+2 \pi j) / 3],
$$

with $j=1,2,3$. Thus, each eigenvalue of the $g$ th generation gives rise to three new eigenvalues, not all of which are different from the previous ones. Therefore, there appear highly degenerate eigenvalues, e.g., the eigenvalue $\lambda=1$ has in the $g$ th generation the degeneracy $\Delta_{g}=(f-2)(f+1)^{g-1}+1$.

Placing now a trap node at the center of the fractal, Volta is able to calculate the (complex) spectrum of the Hamiltonian including the trap [111]. It turns out that only the nondegenerate eigenvalues depend on the trapping strength $\Gamma$. Thus, the degenerate eigenvalues can be again calculated based on the Cardano solution. In particular, these eigenvalues are real and, therefore, do not contribute to the decay of the survival probability. At generation $g$ there are $\left(3^{g}-1\right) / 2+(f+1)^{g}-3^{g}$ 
real degenerate eigenvalues, such that Eq. (107) yields

$$
\begin{aligned}
\Pi_{M}(t) \approx & \frac{1}{N-1}\left[\left(3^{g}-1\right) / 2+(f+1)^{g}-3^{g}\right. \\
& \left.+\sum_{\gamma_{l} \neq 0} \exp \left(-2 \gamma_{l} t\right)\right] .
\end{aligned}
$$

Clearly, in the limit of $t \rightarrow \infty$ this leads to a constant value. As Volta also shows (extending the results for the line), increasing the trapping strength above the value of $\Gamma=V=1$ does not lead to a faster $\Pi_{M}(t)$ decay.

\section{Random networks}

\section{Disordered system with one trap}

Introducing long-range interactions also allows one to study topologically disordered systems. To illustrate this, take a random configuration of $(N-1)$ identical nodes and one trap node [107], see Fig. 37. All $N$ nodes are placed at random in a 3 -dimensional box with Cartesian coordinates $\left\{x^{(i)}\right\}$, with $i=1,2,3$. Then the distance between two nodes $j$ and $k$ is given by

$$
R_{j, k}=\left[\sum_{i=1}^{3}\left(x_{j}^{(i)}-x_{k}^{(i)}\right)^{2}\right]^{1 / 2},
$$

where the coordinates $x_{j}^{(i)}$ and $x_{k}^{(i)}$ are homogeneously distributed random numbers in the interval $[0, N]$. To relate this to the energy transfer dynamics within Rydberg gases one considers interactions decaying as $R_{j, k}^{-3}$. In the absence of traps the corresponding Hamiltonian $\boldsymbol{H}_{0}$ has the following matrix elements

$$
\left\langle k\left|\boldsymbol{H}_{0}\right| j\right\rangle= \begin{cases}-R_{j, k}^{-3} & \text { for } k \neq j \\ \sum_{k \neq j} R_{j, k}^{-3} & \text { for } k=j .\end{cases}
$$

Now, choose one of the $N$ nodes to be a trap, i.e., for this node the full Hamiltonian $\boldsymbol{H}$ has an additional purely imaginary matrix element $-i \Gamma$. Since the configuration of nodes is random, one can (without any loss of generality) assume in the following that the node labeled 1 is the trap.

For disordered systems, one calculates averages over $\mathcal{R}$ different realizations following again, as for the SWN,

$$
\langle\cdot\rangle_{\mathcal{R}} \equiv \frac{1}{\mathcal{R}} \sum_{r=1}^{\mathcal{R}}[\cdot]_{r},
$$

where $[\cdot]_{r}$ denotes the realization $r$. In the calculations one also assumes the trapping strength $[\Gamma]_{r}$ to be realization dependent, because it is required that it be proportional to the diagonal element $\left[\left\langle 1\left|\boldsymbol{H}_{0}\right| 1\right\rangle\right]_{r}$ in that particular realization, namely $[\Gamma]_{r} \equiv \Gamma\left[\left\langle 1\left|\boldsymbol{H}_{0}\right| 1\right\rangle\right]_{r}$. As it turns out, the dependence of the decay on the value of $\Gamma$ is quite weak - different $\Gamma$ mainly rescale the time axis. In Ref. [107] only two extreme

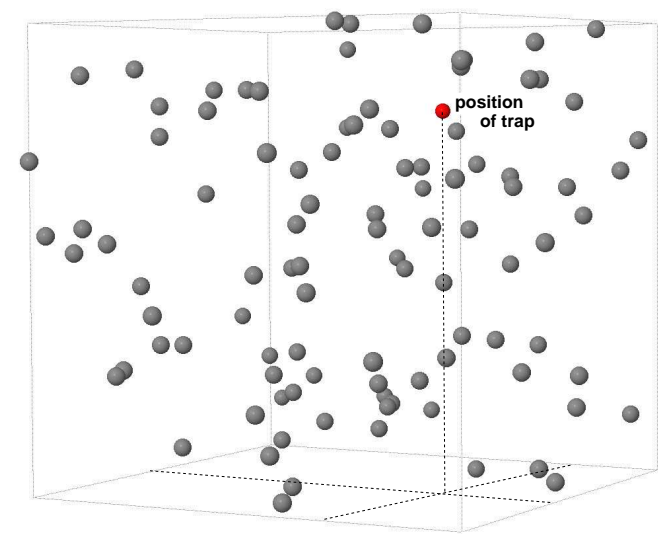

FIG. 37: Random configuration of 99 nodes and a single trap. From [107].

cases were considered: (a) $\Gamma=10^{-6}$, for which a perturbation theoretical treatment can be justified, and (b) $\Gamma=1$, such that the average trapping strength is of the same order as the diagonal elements of $\boldsymbol{H}_{0}$ at the node of the trap.

While each realization $[\Pi(t)]_{r}$ leads to a specific spectrum of the $\left[\gamma_{l}\right]_{r}$, the relation between $\langle\Pi(t)\rangle_{\mathcal{R}}$ and the average $\left\langle\gamma_{l}\right\rangle_{\mathcal{R}}$ is not that straightforward. However, for all $t$, the function $\exp \left(-2 \gamma_{l} t\right)$ is convex, therefore, Jensen's inequality applies, see paragraph 12.41 of [75], such that one obtains for a given $l$ [107]

$$
\left\langle\exp \left(-2 \gamma_{l} t\right)\right\rangle_{\mathcal{R}} \geq \exp \left(-2 t\left\langle\gamma_{l}\right\rangle_{R}\right)
$$

From this one gets a lower bound for $\langle\Pi(t)\rangle_{\mathcal{R}}$ :

$$
\langle\Pi(t)\rangle_{\mathcal{R}} \geq \frac{1}{N} \sum_{l=1}^{N} \exp \left(-2 t\left\langle\gamma_{l}\right\rangle_{\mathcal{R}}\right) .
$$

In Fig. $38\langle\Pi(t)\rangle_{\mathcal{R}}$ is displayed for the two values $\Gamma=$ $10^{-6}$ and $\Gamma=1$, for different $N$. In all cases the intermediate time decay can be fitted by a power-law [107]

$$
\langle\Pi(t)\rangle_{\mathcal{R}} \sim t^{-\eta(N)},
$$

where, different from the regular linear case [50, 105], the exponent $\eta$ is now $N$-dependent. It turns out that approximating the exponent by

$$
\eta(N)=\eta_{0} N^{\mu}
$$

while keeping $\Gamma$ fixed, reproduces the curves well. Note, however, that $\eta_{0}$ and also $\mu$ can still depend on $\Gamma$.

Now, one can estimate $\mu$ based on the results of $\eta$ for $N_{1}=100$ and $N_{2}=1000$. Based on $\eta_{0}=\eta\left(N_{1}\right) / N_{1}^{\mu}=$ $\eta\left(N_{2}\right) / N_{2}^{\mu}$ one has [107]

$$
\mu=\frac{\ln \eta\left(N_{2}\right)-\ln \eta\left(N_{1}\right)}{\ln N_{2}-\ln N_{1}} .
$$

From the numerical values given in Fig. 38 one obtains approximately $\mu \approx-0.166$ and $\eta_{0}=0.0349$ for $\Gamma=10^{-6}$ and $\mu \approx-0.204$ and $\eta_{0}=0.0313$ for $\Gamma=1$. 

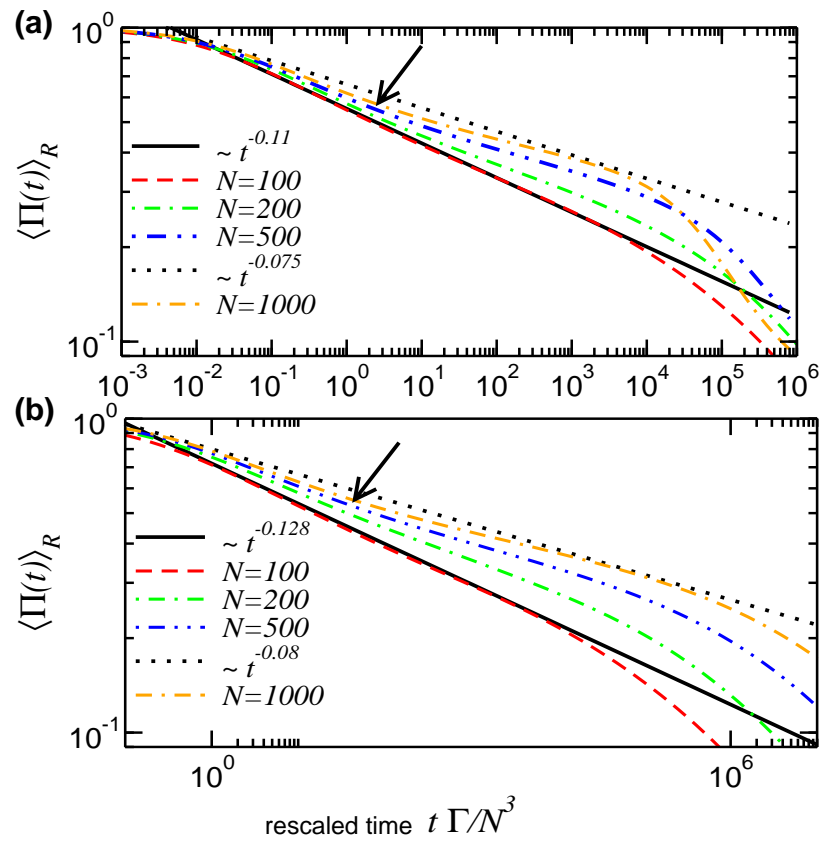

FIG. 38: (Color online) Ensemble averages $\langle\Pi(t)\rangle_{R}$ for different $N$ : (a) for $\Gamma=10^{-6}$ and (b) for $\Gamma=1$. The scalings in the intermediate time regions for $N=100$ and $N=1000$ are shown as solid black lines along with the appropriate scaling law. The arrows are guides to the eye pointing at the bend of $\langle\Pi(t)\rangle_{R}$ for $N=1000$. From [107].

From Fig. 38 as well as from Eq. (139) one sees that the exponent $\eta(N)$ decreases with increasing $N$. Certainly, if $N$ becomes very large it becomes quite improbable (in the ensemble average) for an exciton to encounter the single trap. Therefore, the decay of $\langle\Pi(t)\rangle_{R}$ can only be observed at very long times.

\section{RELATIONS BETWEEN CTRW/CTQW AND OTHER APPROACHES}

\section{A. Phase space approaches}

In order to obtain a unifying framework for describing both quantum mechanical and classical transport one introduces a quantum analog for the classical dynamics, see, e.g. [113]. One particular approach is attributed to Wigner and uses the so-called Wigner-function (WF) in the (quantum mechanical) $2 d$ phase space. If the phase space is spanned by the continuous variables $X$ and $K$, the WF is given by [114, 115]

$$
W(X, K ; t)=\frac{1}{\pi} \int d Y e^{i K Y}\langle X-Y / 2|\hat{\rho}(t)| X+Y / 2\rangle,
$$

where $\hat{\rho}(t)$ is the density operator and thus for a pure state, $\hat{\rho}(t)=|\psi(t)\rangle\langle\psi(t)|$. Here, $\psi(X ; t)=\langle X \mid \psi(t)\rangle$ is the wave function of the particle. The WF is a quasi-probability (in the sense that it can become negative). Integrating $W(X, K ; t)$ along lines in phase space gives marginal distributions, e.g., when integrating along the $K$-axis one has, [114, 115],

$$
\int d K W(X, K ; t)=|\psi(X ; t)|^{2}
$$

In the case of a discrete system, given, for instance, by $N$ discrete positions on a network (enumerated as $0,1, \ldots, N-$ 1 ) the functions $\psi(x)$ are only defined for integer values of $x=0,1, \ldots, N-1$, and the form of Eq.1141 has to be changed from an integral to a sum. There have been several attempts in doing so, see, for instance, [116, 117]. However, the definition of the discrete WFs might depend on whether the length $N$ of the system is even or odd [118, 119].

For a one-dimensional system of length $N$ with periodic boundary conditions (exemplified by a ring) one has $\psi(x) \equiv$ $\psi(x \pm r N)$ for all $r \in \mathbb{N}$. It follows that each and every one of the products $\psi^{*}\left(x-y^{\prime} ; t\right) \psi\left(x+y^{\prime} ; t\right)$ is identical to (at least) one of the $N$ forms $\psi^{*}(x-y ; t) \psi(x+y ; t)$, where $y=0,1, \ldots, N-1$.

Now the WF has the form of a Fourier transform; a unique transformation of these $N$ products requires $N$ different $k$ values. These $k$-values may evidently be chosen as $k=$ $2 \pi \kappa / N$, again having $\kappa=0,1, \ldots, N-1$. One is thus led to propose for integer $x$ and $y$ the following discrete WF [120]

$$
W(x, k ; t)=\frac{1}{N} \sum_{y=0}^{N-1} e^{i k y} \psi^{*}(x-y ; t) \psi(x+y ; t) .
$$

\section{WF for a ring}

Since the eigenstates of the ring are Bloch states, the WF for a CTQW on a ring of $N$ nodes reads [120]

$$
\begin{aligned}
W_{j}(x, \kappa ; t)= & \frac{1}{N^{2}} \sum_{n=0}^{N-1} \exp [-i 2 \pi(2 n+\kappa)(x-j) / N] \\
& \times \exp \{-i 2 t[\cos (2 \pi(\kappa+n) / N) \\
& -\cos (2 \pi n / N)]\}
\end{aligned}
$$

where it has been used that $\theta=2 \pi n / N$ and $k=2 \pi \kappa / N$. Furthermore, one may note from Eq.143) that

$$
\begin{aligned}
& \sum_{\kappa} W_{j}(x, \kappa ; t)=|\psi(x ; t)|^{2} \\
& =\frac{1}{N} \sum_{\kappa} \sum_{y} e^{-i 2 \pi \kappa y / N} \psi^{*}(x-y ; t) \psi(x+y ; t)
\end{aligned}
$$

In general, the WFs have a very complex structure. Figure 39 shows a contour plot of the WF of a CTQW on a cycle of length $N=101$ at different times. Note that at $t=0$ the WF is localized on the strip at the initial point $j$. At $t=1$, the WF is still mostly localized about $j$. As time increases other sites get populated. On short time scales, the WF develops a very regular structure in phase space, with "wavefronts" originating from the initial point $j$. Additionally, one also notes 


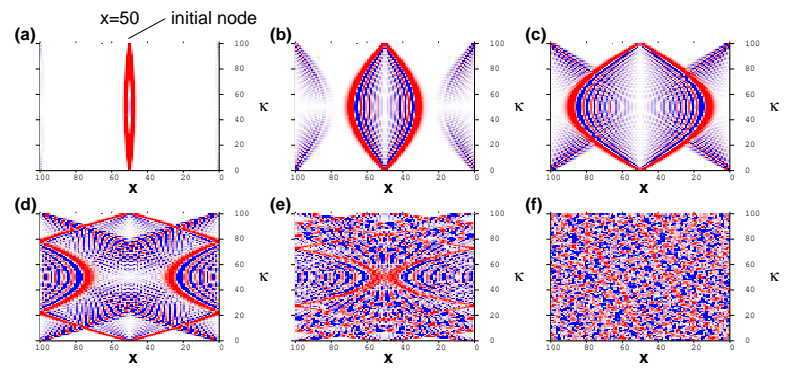

FIG. 39: (Color online) WFs of a CTQW on a cycle of length $N=$ 101 at times $t=1,10,20,40[(\mathrm{a})$-(d)] as well as $t=100,500$ [(e),(f)]. The initial node is at $j=50$. Red regions denote positive values of $W_{j}(x, \kappa ; t)$, blue regions negative values and white regions values close to 0 . From [120].

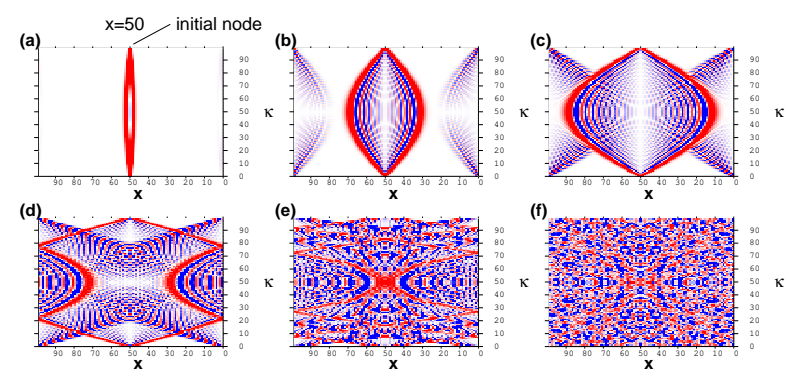

FIG. 40: (Color online) Same as Fig 39, for $N=100$ and $j=50$. From [120].

"wavefronts" starting from the region opposite to the initial point, which are much weaker in amplitude. As time progresses, these two types of waves start interfering with each other.

Figure 40 shows the WF of a CTQW for $N=100$. At short times, the structure of the WF is quite similar to the situation for $N=101$. Nonetheless, there are differences at larger times, visible by comparing Figs 39 (e) and 39(f) to Figs 40(e) and 40 (f).

However, although at long times the interference effects are quite intricate, typical patterns are still visible. At $t=500$, one finds less regularities in phase space for $N=101$ than for $N=100$, reflecting the higher symmetry of CTQW for even $N$. One notes, moreover, that the phase space patterns give a much richer picture of the underlying dynamics than the transition probabilities $\left|\psi_{j}(x ; t)\right|^{2}$ alone.

Furthermore, one should remark that in an infinite system the WFs have a much simpler structure, because there one does not face the problem of distinct wave fronts running into opposite directions and interfering with each other because of the closure of the ring.

In general, the marginal distribution $\sum_{\kappa} W_{j}(x, \kappa ; t)$ is ob- tained from Eq. 144] as [120]

$$
\begin{aligned}
& \sum_{\kappa} W_{j}(x, \kappa ; t)=\frac{1}{N} \sum_{n=0}^{N-1} \exp [i 2 \pi n(x-j) / N] \\
& \times \exp [-i 2 t \cos (2 \pi n / N)] \\
& \times \frac{1}{N} \sum_{\kappa=0}^{N-1} \exp [i 2 \pi(\kappa+n)(x-j) / N] \\
& \times \exp [i 2 t \cos (2 \pi(\kappa+n) / N)] .
\end{aligned}
$$

Since the system is periodic, the arguments $(\kappa+n)$ in the exponentials can be written as $(\kappa+n) \equiv N-\nu$, where $\nu=0,1, \ldots, N-1$. This yields

$$
\begin{aligned}
& \sum_{\kappa} W_{j}(x, \kappa ; t) \\
& =\left|\frac{1}{N} \sum_{n=0}^{N-1} e^{i 2 \pi n(x-j) / N} e^{-i 2 t \cos (2 \pi n / N)}\right|^{2},
\end{aligned}
$$

which is exactly what also follows, see Eq.(145), from calculating $|\psi(x ; t)|^{2}$ directly from the Bloch ansatz, see Eq. (17) and [72].

For the marginal distribution $\sum_{x} W_{j}(x, \kappa ; t)$ one also gets from Eq.(144)

$$
\begin{aligned}
& \sum_{x} W_{j}(x, \kappa ; t)=\frac{1}{N^{2}} \sum_{n=0}^{N-1} N \delta_{2 n,-\kappa} e^{i 2 \pi(2 n+\kappa) j / N} \\
& \times \exp \{-i 2 t[\cos (2 \pi(\kappa+n) / N)-\cos (2 \pi n / N)]\} \\
& = \begin{cases}1 / N & \text { for } N \text { odd and all } \kappa \\
2 / N & \text { for } N \text { even and } \kappa \text { even } \\
0 & \text { for } N \text { even and } \kappa \text { odd }\end{cases}
\end{aligned}
$$

all of these expressions are independent of $t$. Eq. (148) can be confirmed directly by taking the Fourier transform of $|\psi(x ; t)|^{2}$. The whole phase space volume is normalized to unity, as can be seen by summing Eq.(148) over all $\kappa$, with $\kappa=0,1, \ldots, N-1$.

In the long-time average and for odd $N\left(\right.$ superscript $\left.^{\circ}\right)$ most points in the quantum mechanical phase space have a weight of $1 / N^{2}$, namely one has has [103]

$$
\bar{W}_{j}^{o}(x, \kappa)= \begin{cases}1 / N^{2} & \text { for } \kappa \neq 0 \text { and any } x \\ 1 / N & \text { for } \kappa=0 \text { and } x=j \\ 0 & \text { else. }\end{cases}
$$

For even $N$ (superscript ${ }^{e}$ ), the limiting WF reads

$$
\bar{W}_{j}^{e}(x, \kappa)= \begin{cases}2 / N^{2} & \kappa \neq 0, \kappa \text { even and any } x \\ 1 / N & \kappa=0 \text { and } x=j, j+N / 2 \\ 0 & \text { else. }\end{cases}
$$

The long time averages of the WFs for even $N$ are somewhat peculiar, since values different from zero appear only for even 
$\kappa$, whereas the WFs themselves have values different from zero at arbitrary times for all $\kappa$. These stripes in the long-time average are due to the periodicity of the ring. For even $N$ one finds constructive interference patterns in the transition probabilities, since the number of steps in both directions is the same, see also Ref. [72]. For a finite line with even $N$, there are no stripes in the long time average.

\section{Rings with energetic disorder}

By introducing (static) disorder into the system, as in Sec. VB, the Bloch property is lost [103]. In order to have a global picture of the effect of the disorder on the dynamics, one considers ensemble averages of the WFs. For this one calculates the WF for different realizations of $\boldsymbol{H}$ and averages over all realizations, i.e., for $R$ realizations:

$$
\left\langle W_{j}(x, \kappa ; t)\right\rangle_{R} \equiv \frac{1}{R} \sum_{r=1}^{R}\left[W_{j}(x, \kappa ; t)\right]_{r},
$$

where $\left[W_{j}(x, \kappa ; t)\right]_{r}$ is the WF of the $r$ th realization of $\boldsymbol{H}$.

It turns out that the particular type of disorder, i.e., diagonal or diagonal and off-diagonal, does not change the picture significantly. For diagonal and for off-diagonal disorder Figure 41 shows snapshots of $\left\langle W_{j}(x, \kappa ; t)\right\rangle_{R}$ at different times for $N=101$ and for different values of $\Delta$.

The first column shows $\left\langle W_{j}(x, \kappa ; t)\right\rangle_{R}$ for $\Delta=1 / 40$ at times $t=1,10,20,40,100$, and 500 [Fig. 41]1a)-(1f)]. For this quite weak disorder, the patterns in phase space are similar to the unperturbed case, where "waves" in phase space emanate from the initial site $x=j=50$ and start to interfere after having reached the opposite site of the ring (see Fig. 3 of [120]). However, at longer times differences become visible, Fig 41(1f). The pattern for the unperturbed case is quite irregular but with alternating positive and negative regions of the WF of approximately the same magnitude. For $x$ close to the initial site $j=50$, the disorder causes a decrease of $\left\langle W_{j}(x, \kappa ; t)\right\rangle_{R}$ for $\kappa$-values in the middle of the interval $[0, N-1]$ when compared to what is found for values of $\kappa$ close to 0 or to $N-1$.

Increasing the disorder parameter $\Delta$, the patterns change profoundly. The wave structure gets suppressed and for all $\kappa$ a localized region forms about the initial site $j=50$, already for small disorder $(\Delta=1 / 10)$ and short times $(t=20)$, see Fig.412c).

For even larger values of $\Delta$, for all $\kappa$ the formation of a localized region about $j=50$ becomes even more pronounced. Already for $\Delta=1 / 4$ this localized region forms for times as short as $t=10$, see Fig. 41 (3b). At $\Delta=1 / 2,\left\langle W_{j}(x, \kappa ; t)\right\rangle_{R}$ stays localized at all times [Fig. 41(4a)-(4f)]. Also here, the values of the WF at about $\kappa \approx N / 2$ are rather low, whereas the values of the WF for $\kappa$ close to the interval borders 0 and $N-1$ remain rather large for $x \approx j$, as indicated by the thin black region (see also the arrows), e.g., in Fig. 41 (4f). One further notes that at high disorder the localized averaged WF is always positive, i.e., all fluctuations, present for small disorder, have vanished. One recalls that the WF is normalized to
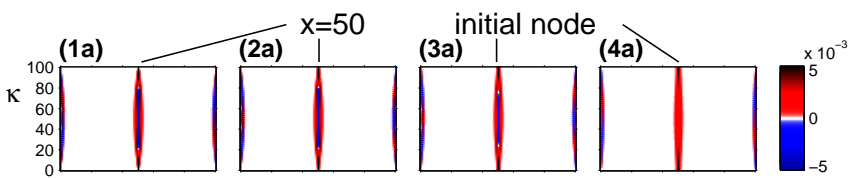

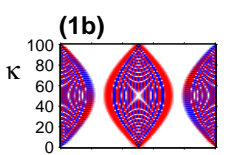

(2b)
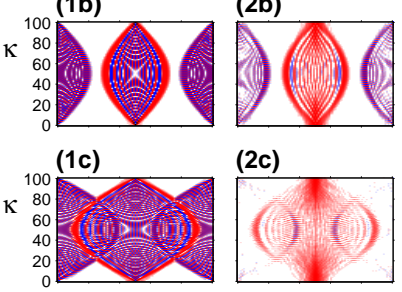

(2c)
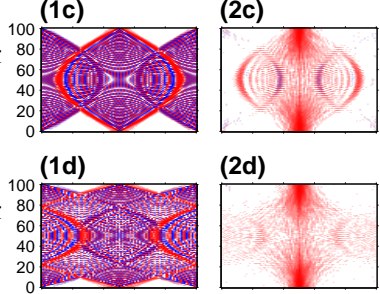

(2d)
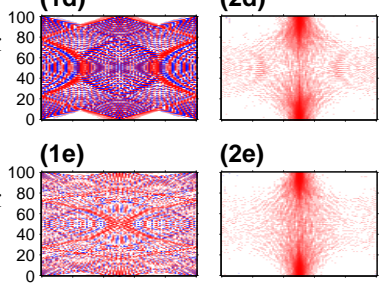

(2e)
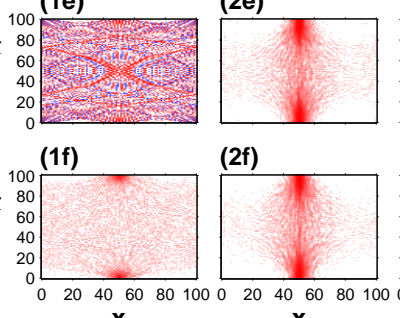

(2f)

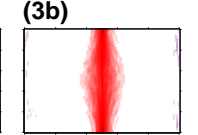

(3c)

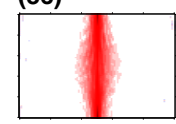

(3d)

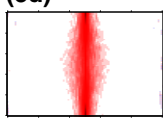

(3e)

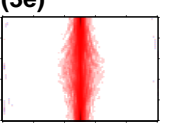

(3f)
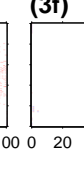

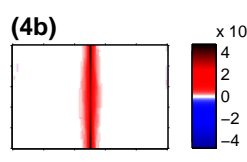

(4c)

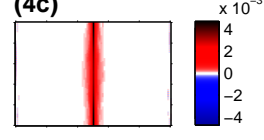

(4d)

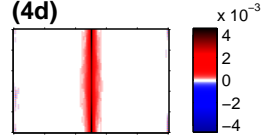

(4e)

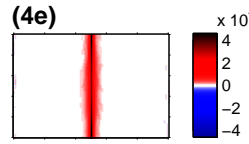

(4f)

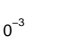

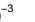

FIG. 41: (Color online) Ensemble average of WFs of the quantum dynamics on a ring of length $N=101$ with diagonal and offdiagonal disorder for $\Delta=1 / 40,1 / 10,1 / 4$, and $1 / 2$ [columns (1)(4)], each at times $t=1,10,20,40,100$, and 500 [rows (a)-(f)]. The initial node is always $j=50$ and the average is over $R=1000$ realizations. Red regions denote positive values of the averaged WFs, blue regions negative values and white regions values close to 0 . The colormaps are always chosen to be the same for each row but might differ in different rows. The maximal values of $\left\langle W_{j}(x, \kappa ; t)\right\rangle_{R}$ are denoted by small black regions; these are highlighted and exemplified by the arrows in panel (4f). From [103].

unity when integrated over the whole phase space. Having an even number of nodes in the graph does not alter the picture significantly.

The ensemble average of the long-time averaged WF follows as [103]

$$
\begin{aligned}
& \left\langle\bar{W}_{j}(x, \kappa)\right\rangle_{R} \equiv\left\langle\lim _{T \rightarrow \infty} \frac{1}{T} \int_{0}^{T} d t W_{j}(x, \kappa ; t)\right\rangle_{R} \\
& =\lim _{T \rightarrow \infty} \frac{1}{T} \int_{0}^{T} d t\left\langle W_{j}(x, \kappa ; t)\right\rangle_{R} .
\end{aligned}
$$

Now, the disorder changes also the limiting WF quite drastically. Starting from high disorder of $\Delta=1 / 2$, one expects from Figs. 41 4a)-(4f) that the long time average of the averaged WF will look basically the same. Figure 42 shows the limiting averaged WF for $N=101$ and DOD according to Eq. (152). Indeed, for large $\Delta$, the limiting averaged WF is comparable to the corresponding averaged WF, compare Figs. 42(4). Close to the initial node $x=j=50$ and 

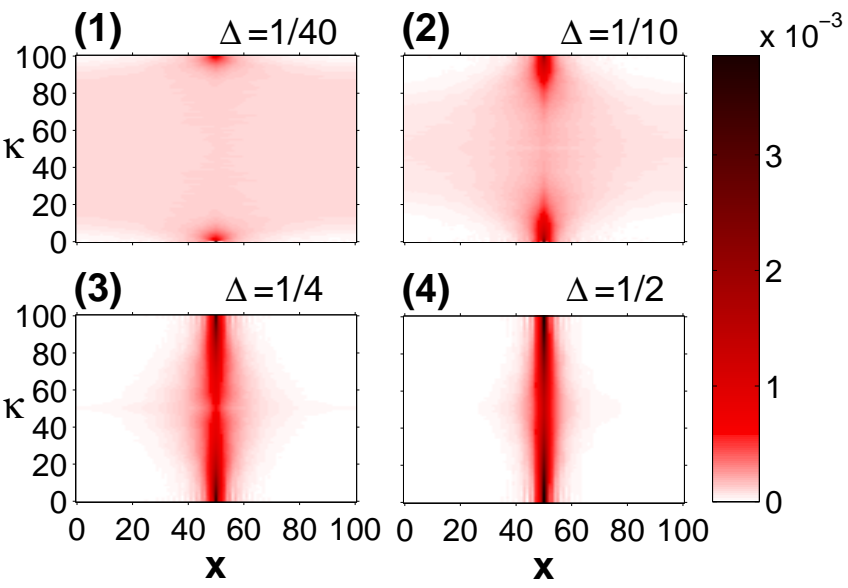

FIG. 42: (Color online) DOD: Limiting averaged WF, $\left\langle\bar{W}_{j}(x, \kappa)\right\rangle_{R}$, for $N=101$ and $\Delta=1 / 40,1 / 10,1 / 4$, and $1 / 2$ [panels (1) to (4), respectively], according to Eq. 152]. From [103].
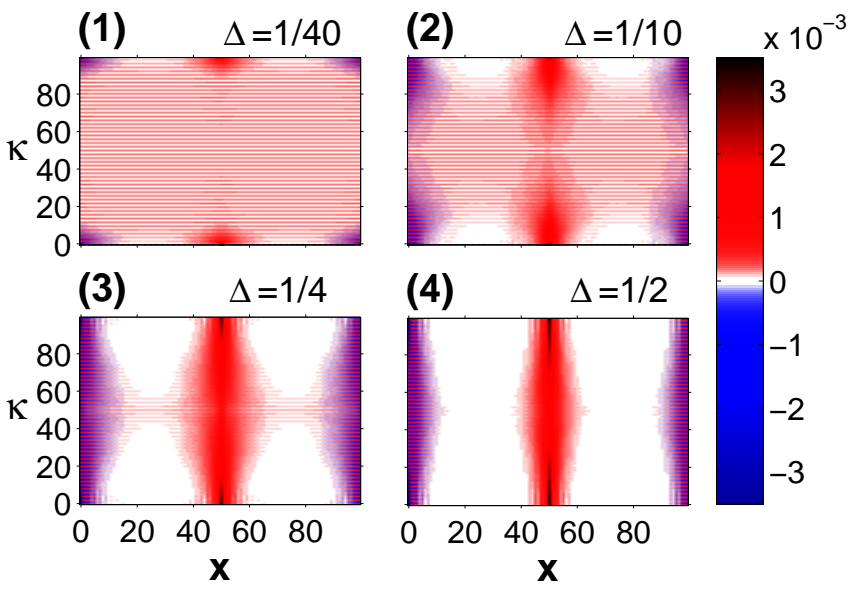

FIG. 43: (Color online) Same as Fig. 42 but for $N=100$. From [103].

there along the $\kappa$-direction, $\left\langle\bar{W}_{j}(x, \kappa)\right\rangle_{R}$ has large (positive) values for $\kappa$ about 0 and $N-1$ which decrease by going toward $\kappa=N / 2$. Here, the decrease depends on the particular type of disorder involved.

Also for small $\Delta$ there are significant differences to the case without disorder. From Fig. 42(1) one sees that the disorder "smears out" the localized value $\bar{W}_{j}(j, 0)=1 / N$ in the absence of disorder, see Eq. (149). Specifically, the $(x=j, \kappa=0)$-value decreases, while the neighboring ones increase. For $\Delta=1 / 10$, the onset of localization about $x=j=50$ can already be seen, e.g., Fig.42(2), and becomes more and more pronounced as $\Delta$ increases, Fig. 42 3 ). Furthermore, all other values of $\left\langle\bar{W}_{j}(x, \kappa)\right\rangle_{R}$ for $x \neq j$ decrease with increasing disorder, as can be seen by the decreasing size of the light pink region, which corresponds to values close to $1 / N^{2}$. In fact, the values of the limiting averaged WF for $x$ distant from $x=j=50$ drop to zero, shown as white regions in Fig. 42 .

For even $N$ one finds that without disorder the limiting WF shows a peculiar "striped" distribution, caused by the PBCs, see Eq. (150). By switching on the disorder, these peculiarities vanish. Figure 43 shows the limiting averaged WF for DOD and $N=100$. Although for small $\Delta$ there are some remainders of stripes left, these disappear completely for higher values of $\Delta$, compare Figs. 43(1)-(4). Note further that the second peak of $\bar{W}_{j}^{e}(x, \kappa)$ at $x=j+N / 2$, see Eq. 150), transforms for increasing disorder to an oscillatory line in the $\kappa$-direction at $x=j+N / 2$.

\section{Long-range interaction cycles}

$\mathrm{Xu}$ and Liu have considered the WF, defined in Eq. (143) and [120], for rings, where the $m$ next nearest neighbors of a given node are connected to this node by bonds [121], see also Sec. VA. The eigenstates of such a translationally invariant system are still Bloch states. However, the eigenvalues read [121]

$$
E_{n}=2 m-2 \sum_{j=1}^{m} \cos 2 n j \pi / N .
$$

As in Eq. 144), the WF follows as

$$
\begin{aligned}
W_{j}(x, \kappa: t)= & \frac{1}{N^{2}} \sum_{n=0}^{N-1} e^{-i t\left(E_{n}-E_{N+\kappa-n}\right)} \\
& \times e^{i 2 \pi(2 n-\kappa)(x-j) / N}
\end{aligned}
$$

Thus, inserting the results for the eigenvalues one obtains the WF for this type of long-range interacting cycles.

Now, while for $m=1$ one recovers the results of the previous section, increasing $m$ leads to more complex structures of the WF. The fronts emanating from the initial value $x=j$ have, in the case of $m=1$, only a single maximum value at about $\kappa=N / 2$, see Figs. 39 and 40. For $m>1$ there appear $m$ front maxima at about $\kappa=(2 l-1) N / 2 m$ with $l=1, \ldots, m[121]$. With increasing number $m$ the structure of the WFs become more complex, but the resulting patterns can still be attributed to the interfering fronts emanating from $x=j[121]$.

\section{Disordered networks}

$\mathrm{Xu}$ and Liu have also studied the WF for disordered systems, namely for the Watts-Strogatz (WS) small-world model [121]. The WS model starts from an ordered ring with only nearest neighbor interactions. Now, every bond is rewired with probability $p$, implying that for $p=0$ one retains the ordered ring and for $p=1$ one has a completely disordered network of $N$ nodes with $N$ bonds. The WF for such systems shows essentially the same features as for rings with energetic disorder, see above. In the ensemble average there is stronger and stronger localization with increasing $p$. Moreover, here also the distinction between even and odd $N$ remains, namely even $N$ lead to a stripe-patterned WF caused by constructive interference while odd $N$ do not show this behavior [121]. 


\section{B. Quantum master equations}

CTQW and CTRW are the two extreme cases of possible transport processes, i.e., a purely coherent transport for CTQW and a purely incoherent transport for CTRW. Now, if a quantum mechanical system cannot be regarded as being isolated from its environment, this environment will influence the dynamics of the system. Depending on the strength of the coupling (where also the temperature is assumed to be controlled by the environment), the dynamics may change from quantal to classical. Since the total system (i.e., the system $S$ and the environment/reservoir $R$ ) resides in a Hilbert space of huge dimension (this due to the many degrees of freedom (DOF) of the environment), one often considers the system only, where the environmental DOF have been traced out [69].

Let $\boldsymbol{W}(t)$ be the density operator of the total system, then $\boldsymbol{\rho}(t) \equiv \operatorname{tr}_{R}\{\boldsymbol{W}(t)\}$ is called the reduced density operator. Then the dynamics of $\boldsymbol{W}(t)$ is still governed by Schrödinger's equation, which can be recast into the Liouville-von Neumann ( $\mathrm{LvN}$ ) equation

$$
\dot{\boldsymbol{W}}(t)=-i\left[\boldsymbol{H}_{\mathrm{tot}}, \boldsymbol{W}(t)\right],
$$

where $\boldsymbol{H}_{\text {tot }}$ is the Hamiltonian of the total system. $\boldsymbol{H}_{\text {tot }}$ usually comprises three parts: the Hamiltonians $\boldsymbol{H}_{S}$ for the system, $\boldsymbol{H}_{R}$ for the environemnt, and $\boldsymbol{H}_{S R}$ for the coupling between system and environment:

$$
\boldsymbol{H}_{\text {tot }}=\boldsymbol{H}_{S}+\boldsymbol{H}_{R}+\boldsymbol{H}_{S R}
$$

Inserting this into the LvN equation for $\boldsymbol{W}(t)$ and integrating out the environmental DOF one arrives at an equation for the reduced density operator (for details see [69]):

$$
\dot{\boldsymbol{\rho}}(t)=-i\left[\boldsymbol{H}_{S}, \boldsymbol{\rho}(t)\right]+\mathcal{D}[\boldsymbol{\rho}(t)] .
$$

Here, $\mathcal{D}[\boldsymbol{\rho}(t)]$ is called the dissipator. Note that in order to arrive at Eq. 157) several assumptions, such as bilinear couplings between system and environment or the Markov approximation, have been used [69].

Under certain conditons, such as a weak coupling between the system and the environment, the dissipator can also be expressed by operators acting on the Hilbert space of the system $S$. In particular, Eq. 157) can be brought into the so-called Lindblad form [69]

$$
\begin{aligned}
\dot{\boldsymbol{\rho}}(t)= & -i\left[\boldsymbol{H}_{S}, \boldsymbol{\rho}(t)\right]-\sum_{j=1}^{N^{2}}\left(2 \boldsymbol{L}_{j} \boldsymbol{\rho}(t) \boldsymbol{L}_{j}^{\dagger}\right. \\
& \left.-\boldsymbol{L}_{j}^{\dagger} \boldsymbol{L}_{j} \boldsymbol{\rho}(t)-\boldsymbol{\rho}(t) \boldsymbol{L}_{j}^{\dagger} \boldsymbol{L}_{j}\right),
\end{aligned}
$$

where the operators $\boldsymbol{L}_{j}$, the Lindblad operators, mimick the influence of the environemnt on the dynamics. Equation (158) is also called Lindblad quantum master equation (LQME).

Obviously, if the coupling to the environment vanishes, one arrives again at the $\mathrm{LvN}$ equation for the density operator $\boldsymbol{\rho}(t)$ of the system $S$. Since the resulting LvN equation is equivalent to the Schrödinger equation of a closed system with Hamiltonian $\boldsymbol{H}$, it is also equivalent to the CTQW formulation of the previous sections. Now, when increasing the coupling to the environment, Eq. (158) allows to study the onset of the quantum to classical cross-over.

\section{Decoherence on rings}

Take now a ring of $N$ nodes and $N$ Lindblad operators each of which projects onto a single node, i.e., $\boldsymbol{L}_{j}=\sqrt{\lambda_{j}}|j\rangle\langle j|$ for $j=1, \ldots, N$. The parameters $\lambda_{j}$ specify the strength of the coupling to the environment. If $\lambda_{j}=\lambda$ for all $j$, Eq. (158) simplifies:

$$
\begin{aligned}
\dot{\boldsymbol{\rho}}(t)= & -i\left[\boldsymbol{H}_{S}, \boldsymbol{\rho}(t)\right] \\
& -2 \lambda \sum_{j}\left(\boldsymbol{\rho}(t)-\rho_{j j}(t)\right)|j\rangle\langle j|,
\end{aligned}
$$

where $\rho_{k j}(t) \equiv\langle k|\boldsymbol{\rho}(t)| j\rangle$. The rate $\lambda$ can be estimated from the spectral density $J(\omega)$ describing the environment within the Caldeira-Leggett model [122, 123] at a given temperature $T$. Taking $J(\omega)=2 \pi \alpha \omega \exp \left(-\omega / \omega_{c}\right)$ and using the Markov approximation one arrives at $\lambda=\pi \alpha k_{B} T$ [69]. One has to bear in mind that Eq. (159) is an approximation with a limited range of validity: For a very large coupling strength $\lambda$, Eq. (170) leads to the quantum Zeno limit rather than to a classical master/rate equation. In matrix form Eq. (159) is equivalent to the so-called Gurvitz model, see for instance [124], considered in 125-127]

$$
\begin{aligned}
\dot{\rho}_{k j}(t)= & -i\left\langle k\left|\left[\boldsymbol{H}_{S}, \boldsymbol{\rho}(t)\right]\right| j\right\rangle \\
& -2 \lambda\left(1-\delta_{k j}\right) \rho_{k j}(t) .
\end{aligned}
$$

Solenov and Fedichkin showed analytically that the probability distribution to be at node $k$, where for a ring one assumes without loss of generality that the initial node is $j=0$, follows from the diagonal elements of the reduced density matrix [126, 127]

$$
\begin{aligned}
& \rho_{k, k}(t)=\frac{1}{N}+\sum_{n, m=0}^{N-1} \frac{1-\delta_{n+m, 0}-\delta_{n+m, N}}{N^{2}} \\
& \times\left[\delta_{n, m} e^{-2 \lambda[(N-1) / N] t}+\left(1-\delta_{n, m}\right) e^{-2 \lambda[(N-2) / N] t}\right] \\
& \times \exp \left[i t \sin \frac{\pi(n+m)}{N} \cos \frac{\pi(n-m)}{N}\right. \\
& \left.+\frac{2 \pi i}{N}(n+m) k\right] .
\end{aligned}
$$

Clearly, Eq. (161) reduces to the results of Sec. IV A in the limit of $\lambda \rightarrow 0$. For $\lambda \neq 0$, due to the exponentially decaying factors, the probability distribution will eventually decay to the classical equilibrium values, in this case the equipartition value $1 / N$.

The so-called mixing time $t_{m}$ is used to compare the spreading of CTQW to that of CTRW. It is implicitly defined by looking at the deviation from the classical uniform distribution $1 / N$ [125]. One gets thus

$$
\sum_{k=0}^{N-1}\left|\rho_{k, k}\left(t_{m}\right)-\frac{1}{N}\right| \leq \varepsilon,
$$

where $\varepsilon$ is a small dimensionless constant representing the degree of mixing. By using Eq. (161), Solenov and Fedichkin 
showed that the mixing time is bound from above by

$$
t_{\mathrm{mix}} \equiv \min t_{m}<\frac{N}{2 \lambda(N-2)} \ln \left(\frac{N+1}{\varepsilon}\right) .
$$

The same authors have generalized this approach to socalled hypercycles. A hypercycle of dimension $d$ and size $N$ is build by considering $N$ copies of the $d-1$ dimensional hypercycle and connecting the corresponding nodes, see [125] for details. Thereby, the total number of nodes is $N^{d}$. The corrsponding upper bound for the mixing time $t_{m}$ now follows as

$$
t_{\text {mix }}^{(d)} \leq \frac{d}{2 \lambda} \frac{N}{N-1} \ln \left[\frac{d(N+1)\left(1+\varepsilon N^{d}\right)}{\varepsilon}\right] .
$$

Salimi and Radgohar have considered the long-range case of the single cycle, where a given node interacts with its $2 m$ nearest neighbors ( $m$ to each side) [128], see also Sec. VA. The probability distribution to be at node $k$ is then obtained for even $m$ as

$$
\begin{aligned}
& \rho_{k, k}(t)=\frac{1}{N}+\sum_{n, l=0}^{N-1} \frac{1-\delta_{n+l, 0}-\delta_{n+l, N}}{N^{2}} \\
& \times \exp [-2 \lambda[(N-1) / N] t] \\
& \times \exp \left[i t \sin \frac{\pi(n+l)}{N} \cos \frac{\pi(n-l)}{N}+\frac{2 \pi i}{N}(n+l) k\right] \\
& \times \exp \left[i^{m+1} t \sin \frac{\pi m(n+l)}{N} \sin \frac{\pi m(n-l)}{N}\right] .
\end{aligned}
$$

and for odd $m$ as

$$
\begin{aligned}
& \rho_{k, k}(t)=\frac{1}{N}+\sum_{n, l=0}^{N-1} \frac{1-\delta_{n+l, 0}-\delta_{n+l, N}}{N^{2}} \\
& \times\left[\delta_{n, l} e^{-2 \lambda[(N-1) / N] t}+\left(1-\delta_{n, l}\right) e^{-2 \lambda[(N-2) / N] t}\right] \\
& \times \exp \left[i t \sin \frac{\pi(n+l)}{N} \cos \frac{\pi(n-l)}{N}+\frac{2 \pi i}{N}(n+l) k\right] \\
& \times \exp \left[i^{m+1} t \sin \frac{\pi m(n+l)}{N} \sin \frac{\pi m(n-l)}{N}\right] .
\end{aligned}
$$

While the upper bounds of the mixing time turn out to be independent of $m$, they do differ for even and for odd $m$, one has namely

$$
t_{\text {mix }} \leq \frac{N}{N-1} \ln \frac{N}{\varepsilon} \quad \text { for even } m
$$

and

$$
t_{\text {mix }} \leq \frac{N}{N-2} \ln \frac{N}{\varepsilon} \quad \text { for odd } m .
$$

Therefore, mixing turns out to be faster for even $m$ than for odd $m$.

\section{Dimer with traps}

Within the phenonmenological approach presented above, introducing traps into the system leads to non-Hermitian Hamiltonians. The LvN equation changes, therefore, to

$$
\dot{\boldsymbol{W}}(t)=-i\left[\boldsymbol{H}_{0}, \boldsymbol{W}(t)\right]-\{\boldsymbol{\Gamma}, \boldsymbol{W}(t)\},
$$

where $\{\cdot, \cdot\}$ is the anti-commutator and $\boldsymbol{H}_{0}$ the Hamiltonian without traps. Writing the Lindblad operators in the form $\sqrt{\lambda} \boldsymbol{L}_{j}$, the LQME reads [129]

$$
\begin{aligned}
\dot{\boldsymbol{\rho}}(t)= & -i\left[\boldsymbol{H}_{0}, \boldsymbol{\rho}(t)\right]-\{\boldsymbol{\Gamma}, \boldsymbol{\rho}(t)\} \\
& -2 \lambda \sum_{j}\left(\boldsymbol{\rho}(t)-\rho_{j j}(t)\right) \boldsymbol{L}_{j} .
\end{aligned}
$$

Now, consider a dimer which is coupled to an external bath. This situation allows to solve Eq. (170) analytically and to compare the LQME results to the numerically exact Path Integral Monte Carlo (PIMC) calculations. For details on PIMC techniques see, e.g., [130, 131] and references therein. The Hamiltonian of the dimer without any coupling to the surroundings can be expressed through the Pauli matrices $\sigma_{z}$ and $\sigma_{x}$,

$$
\boldsymbol{H}=E \mathbf{1}-V \boldsymbol{\sigma}_{x}-i \frac{\Gamma}{2}\left(\mathbf{1}-\boldsymbol{\sigma}_{z}\right),
$$

where $E$ is the on-site energy, which is choosen to be the same for both nodes, and $V$ is the coupling between the two nodes. It is easily verified that the eigenvalues are [129]

$$
E_{ \pm}=E \pm V e^{ \pm i \phi}=E \pm \sqrt{V^{2}-\Gamma^{2} / 4}-i \Gamma / 2
$$

where $\phi=\arcsin (\Gamma / 2 V)$. For $\Gamma \rightarrow 0(\phi \rightarrow 0)$ this yields the correct eigenvalues $E \pm V$ of $\boldsymbol{H}_{0}$. Note that for $\Gamma \leq$ $2 V$ the negative imaginary part of $E_{ \pm}$is identical for both eigenvalues, i.e., $\gamma_{+}=\gamma_{-}=\Gamma / 2$. The bi-orthonormalized eigenstates of $\boldsymbol{H}$ are of the form [129]

$$
\left|\Phi_{ \pm}\right\rangle \equiv \frac{1}{\sqrt{2 \cos \phi}}\left(\begin{array}{c}
e^{ \pm i \phi / 2} \\
\pm e^{\mp i \phi / 2}
\end{array}\right)
$$

and

$$
\left|\tilde{\Phi}_{ \pm}\right\rangle \equiv \frac{1}{\sqrt{2 \cos \phi}}\left(\begin{array}{c}
e^{\mp i \phi / 2} \\
\pm e^{ \pm i \phi / 2}
\end{array}\right)
$$

where the phases $\phi$ depend on $\Gamma$, such that in the limit $\Gamma \rightarrow 0$ one recovers the eigenstates of $\boldsymbol{H}_{0}$.

When the coupling to the environment vanishes $(\lambda \rightarrow 0)$, one obtains the survival probability directly from the eigenstates and eigenvalues of $\boldsymbol{H}$. For $\Gamma \leq 2 V$ one has [129]

$$
\Pi(t)=e^{-\Gamma t} \frac{\cos ^{2}(\phi+t V \cos \phi)}{\cos ^{2} \phi} \quad(\text { for } \lambda=0) .
$$

Note that for values $\Gamma>2 \mathrm{~V}$ the dimer is overdamped. 
When considering the dimer without traps $(\Gamma=0)$ but coupled to the environment, Eq. (170) simplifies and, from the solution for $\rho$, one obtains the transition probabilities [129]

$$
\begin{aligned}
\pi_{1,1}^{(0)}(t)= & \frac{1}{2}+\frac{e^{-\lambda t}}{2}\left[\frac{\lambda \sin \left(t \sqrt{4 V^{2}-\lambda^{2}}\right)}{\sqrt{4 V^{2}-\lambda^{2}}}\right. \\
& \left.+\cos \left(t \sqrt{4 V^{2}-\lambda^{2}}\right)\right] \quad(\text { for } \Gamma=0) .
\end{aligned}
$$

Moreover, $\pi_{2,1}^{(0)}(t)=1-\pi_{1,1}^{(0)}(t)$. From Eq. (176) one recovers for $\lambda \rightarrow 0$ the simple oscillatory behavior of the transition probabilities [namely, $\lim _{\lambda \rightarrow 0} \pi_{1,1}^{(0)}(t)=\cos ^{2}(V t)$ ]. When the coupling to the surroundings does not vanish, for $\lambda>0$, the transition probabilities still show oscillations, which are superimposed on an exponential decay which tends for long times to the classical equipartition value of $1 / 2$.

In order to combine the results of Eq. (175) - taking values $\Gamma<2 V-$ and Eq. (176), one expands in both equations all terms except the exponentials to first order in $\Gamma$ and $\lambda$, respectively. Note that for Eq. (175) one obtains a product of $\exp (-\Gamma t)$ and $\cos ^{2} V t$, which is the simple oscillatory behavior of the dimer in the absence of the trap. Now, the coupling to the environment affects all transitions but still conserves probabilities. Therefore, one replaces the term $\cos ^{2} V t$ by the expansion of Eq. (176), such that one obtains [129]

$$
\begin{aligned}
\Pi(t) & \approx e^{-\Gamma t} \pi_{1,1}^{(0)}(t) \\
& \approx e^{-\Gamma t}\left[\frac{1}{2}+\frac{e^{-\lambda t}}{2}\left(\cos 2 V t+\frac{\lambda}{2 V} \sin 2 V t\right)\right] .
\end{aligned}
$$

Figure 44 compares the survival probabilities of a dissipative dimer obtained from the approximative LQME approach to the numerically exact PIMC calculations for a bath with ohmic spectral density with exponential cutoff, $J(\omega)=$ $2 \pi \alpha \omega e^{-\omega / \omega_{c}}$. The initial condition is $\pi_{1,1}(0)=1$, i.e., at $t=0$ the system is localized in the non-trap node 1 of the dimer. Here, the on-site energies $E$ and the coupling elements $V$ have been taken to be equal, $E=V=1$, one sets $\omega_{c}=5 \mathrm{~V}$ and the temperature is fixed to $k_{B} T=V$.

For small trapping strength $(\Gamma=0.1)$ and vanishing coupling to the environment $(\alpha=0)$, Fig. 44(a), the PIMC calculations coincide with the result of Eq. (175). A moderate increase of the coupling $(\alpha=1 / 10)$, Fig. 44(b), still leads for Eqs. (170) (solid lines) and (177) (dashed lines) to results which are in excellent agreement with the findings of the PIMC calculations (symbols). When increasing the coupling further to $\alpha=1 / 4$, Fig. 44(c), however, the approximate solution, Eq. (177), begins to deviate from the LQME and the PIMC calculations, which are still in very good agreement [129].

As the numerical effort of real-time PIMC simulations grows exponentially with time, they can cover only short-tointermediate time scales. However, the agreement between the LQME and the PIMC calculations in the weak coupling
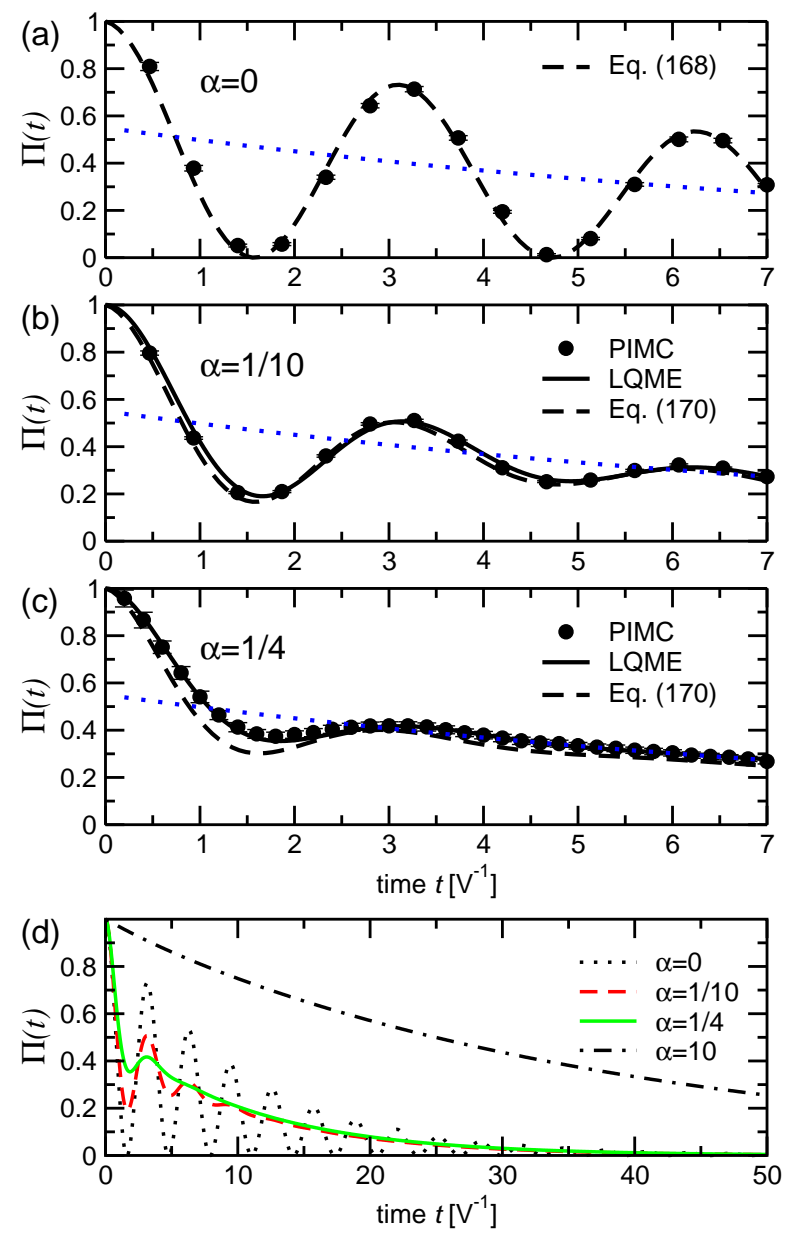

FIG. 44: (Color online) PIMC results (circles) for a dimer with $\Gamma=0.1$ and different system-bath couplings $\alpha=\lambda / \pi$ : (a) $\alpha=0$, (b) $\alpha=1 / 10$, and (c) $\alpha=1 / 4$. The solid lines represent the numerical solution of the LQME equation, the dashed lines show the corresponding analytical results obtained from Eq. (175) for $\alpha=\lambda=0$ and from Eq. 177) for $\alpha=1 / 10$ and $\alpha=1 / 4$. The dotted blue line shows the long time limit $\Pi(t) \sim \exp (-\Gamma t)$. Panel (d) shows the corresponding long-time behavior of the numerical LQME solution for the three different values of $\alpha$ and additionally the behavior for large couplings $\alpha=10$. From [129].

regime permits to compensate for this shortcoming; it allows namely to use for longer times the LQME procedure, see Fig. 44(d).

\section{OUTLOOK}

The application of CTQW to transport for large classes of (physical, chemical, and biological) phenomena involving different types of networks has turned out to be very successful in recent years. However, only little is known about the detailed relations between topology, interaction ranges, dimensions and the transport efficiencies. While CTRW fall into different universality classes depending on the (fractal) dimensions and the interaction ranges, there are only few indica- 
tions on the analogous emergence of "quantum universality classes". Take as an example the dynamics on a chain with long-range interactions decaying as $|k-j|^{-\gamma}$, see Sec. VA, in this case the mean square displacement of CTQW increases as $t^{2}$ also for $\gamma=2$. This is not the case for CTRW, where only systems with $\gamma>3$ lead to the same power-law behavior of the mean square displacement.

Therefore, a thorough investigation of the influence of different topological aspects on the dynamics is clearly necessary. Moreover, once a systematic classification of CTQW gets established, one needs to examine the transition from the quantum to the classical picture, in order to understand the changes in the universality patterns. For this, the environment of each quantum system has to be properly taken into account. In a phenomenological ansatz one can incorporate this either by using the quantum master equation approach, sketched in Sec.VIIB, or by using so-called generalized master equations (GME) [8]. Here, the Markovian master equation (as used in CTRW) is extended by a memory-kernel (MK) to account for non-Markovian effects. In the limit of a MK of delta-type one recovers the CTRW, while in the limit of a constant MK one arrives for CTQW at a wave equation similar to the Schrödinger equation. Both approaches allow to study the influence of the environment on the dynamics. Clearly, the validity of both methods has to be checked. One option to do so is to compare the results - for not too large systems - to those of numerically exact PIMC simulations.

Such a comparison has several advantages. First, it sets the range of validity of the phenomenological approaches. Second, once this range is established, it allows to extend the short-time PIMC results to (in principle) arbitrary long times. Thus, joining different methods together may lead to a deeper understanding of the complex transport processes found in nature.

\section{Acknowledgements}

Support from the Deutsche Forschungsgemeinschaft (DFG), the Fonds der Chemischen Industrie and the Ministry of Science, Research and the Arts of Baden-Württemberg (AZ: 24-7532.23-11-11/1) is gratefully acknowledged. We thank Elena Agliari, Veronika Bierbaum, Lothar Mühlbacher, Volker Pernice, Tobias Schmid, and Antonio Volta for many fruitful discussions.
[1] J.-P. Bouchaud, A. Georges, Phys. Rep. 195 (1990) 127.

[2] R. Albert, A.-L. Barabási, Rev. Mod. Phys. 74 (2002) 47.

[3] S. N. Dorogovtsev, J. F. F. Mendes, Adv. Phys. 51 (2002) 1079.

[4] J. Kempe, Contemporary Physics 44 (2003) 307.

[5] N. van Kampen, Stochastic Processes in Physics and Chemistry, North-Holland, Amsterdam, 1990.

[6] G. H. Weiss, Aspects and Applications of the Random Walk, North-Holland, Amsterdam, 1994.

[7] J. M. Ziman, Principles of the Theory of Solids, Cambridge University Press, Cambridge, England, 1972.

[8] V. M. Kenkre, P. Reineker, Exciton Dynamics in Molecular Crystals and Aggregates, Springer, Berlin, 1982.

[9] N. W. Ashcroft, N. D. Mermin, Solid State Physics, Saunders College Publishing, Philadelphia, 1976.

[10] C. Kittel, Introduction to Solid State Physics, Wiley, New York, 1986.

[11] P. W. Anderson, Phys. Rev. 109 (1958) 1492.

[12] D. A. McQuarrie, Quantum Chemistry, Oxford University Press, Oxford, 1983.

[13] M. Doi, S. F. Edwards, The Theory of Polymer Dynamics, Oxford University Press, Oxford, 1998.

[14] H. Scher, M. Lax, Phys. Rev. B 7 (1973) 4491.

[15] H. Scher, E. W. Montroll, Phys. Rev. B 12 (1975) 2455.

[16] S. Alexander, R. Orbach, J. Phys. (Paris) Lett. 43 (1982) L625.

[17] J. Klafter, A. Blumen, J. Chem. Phys. 80 (1984) 875.

[18] U. Even, K. Rademann, J. Jortner, N. Manor, R. Reisfeld, Phys. Rev. Lett. 52 (1984) 2164.

[19] J. Klafter, A. Blumen, M. F. Shlesinger, Phys. Rev. A 35 (1987) 3081.

[20] G. Zumofen, A. Blumen, J. Klafter, M. Shlesinger, J. Stat. Phys. 54 (1989) 1519.

[21] E. Farhi, S. Gutmann, Phys. Rev. A 58 (1998) 915.

[22] Y. Aharonov, L. Davidovich, N. Zagury, Phys. Rev. A 48
(1993) 1687.

[23] O. Mülken, A. Blumen, Phys. Rev. E 71 (2005) 016101.

[24] S. Bose, Phys. Rev. Lett. 91 (2003) 207901.

[25] F. W. Strauch, Phys. Rev. A 74 (2006) 030301(R).

[26] M. A. Nielsen, I. L. Chuang, Quantum Computation and Quantum Information, Cambridge University Press, Cambridge, England, 2000.

[27] P. W. Shor, in: 35th IEEE Symposium on Foundations of Computer Science, IEEE, Los Alamos, 1994, p. 124.

[28] L. K. Grover, Phys. Rev. Lett. 79 (2) (1997) 325.

[29] A. M. Childs, J. Goldstone, Phys. Rev. A 70 (2004) 022314.

[30] E. Agliari, A. Blumen, O. Mülken, Phys. Rev. A 82 (2010) 012305.

[31] R. P. Feynman, Int. J. Theor. Phys. 21 (1982) 467.

[32] D. A. Meyer, J. Stat. Phys. 85 (1996) 551.

[33] M. Christandl, N. Datta, A. Ekert, A. J. Landahl, Phys. Rev. Lett. 92 (2004) 187902.

[34] T. Kottos, U. Smilansky, Phys. Rev. Lett. 79 (1997) 4794.

[35] H. Schanz, U. Smilansky, Phys. Rev. Lett. 84 (2000) 1427.

[36] T. Kottos, U. Smilansky, Phys. Rev. Lett. 85 (2000) 968.

[37] T. Kottos, H. Schanz, Phys. Rev. Lett. 90 (2003) 234101.

[38] U. Smilansky, J. Phys. A 40 (2007) F621.

[39] J. Sakurai, Modern Quantum Mechanics, 2nd Edition, Addison-Wesley, Redwood City, CA, 1994.

[40] W. Kinzel, Phys. B1. 51 (1995) 1190.

[41] F. Grossmann, J.-M. Rost, W. P. Schleich, J. Phys. A 30 (1997) L277.

[42] M. V. Berry, J. Phys. A 29 (1996) 6617.

[43] I. Bloch, Nature Physics 1 (2005) 23.

[44] B. C. Sanders, S. D. Bartlett, B. Fregenna, P. L. Knight, Phys. Rev. A 67 (2003) 042305.

[45] R. Côté, A. Russel, E. E. Eyler, P. L. Gould, New J. Phys. 8 (2006) 156.

[46] W. Dür, R. Raussendorf, V. M. Kendon, H.-J. Briegel, Phys. 
Rev. A 66 (2002) 052319.

[47] P. L. Knight, E. Roldán, J. E. Sipe, Opt. Comm. 227 (2003) 147.

[48] P. Zhang, X.-F. Ren, X.-B. Zou, B.-H. Liu, Y.-F. Huang, G.-C. Guo, Phys. Rev. A 75 (2007) 052310.

[49] H. B. Perets, Y. Lahini, F. Pozzi, M. Sorel, R. Morandotti, Y. Silberberg, Phys. Rev. Lett. 100 (2008) 170506.

[50] O. Mülken, A. Blumen, T. Amthor, C. Giese, M. ReetzLamour, M. Weidemüller, Phys. Rev. Lett. 99 (2007) 090601.

[51] D. Burgarth, Quantum state transfer with spin chains, Ph.D. thesis, University College London (2006).

[52] S. Bose, Contemp. Phys. 48 (2007) 13.

[53] W. R. Anderson, J. R. Veale, T. F. Gallagher, Phys. Rev. Lett. 80 (1998) 249.

[54] I. Mourachko, C. Comparat, F. de Tomasi, A. Fioretti, P. Nosbaum, V. Akulin, P. Pillet, Phys. Rev. Lett. 80 (1998) 253.

[55] S. Westermann, T. Amthor, A. L. de Oliveira, J. Deiglmayr, M. Reetz-Lamour, M. Weidemüller, Eur. Phys. J. D 40 (2006) 37.

[56] G. S. Engel, T. R. Calhoun, R. L. Read, T.-K. Ahn, T. Manal, Y.-C. Cheng, R. E. Blankenship, G. R. Fleming, Nature 446 (2007) 782.

[57] E. Collini, C. Y. Wong, K. E. Wilk, P. M. G. Curmi, P. Brumer, G. D. Scholes, Nature 463 (2010) 644.

[58] D.-J. Heijs, V. A. Malyshev, J. Knoester, J. Chem. Phys. 121 (2004) 4884.

[59] S. M. Vlaming, D. J. Heijs, J. Knoester, J. Lumin. 111 (2005) 349.

[60] A. Blumen, A. Volta, A. Jurjiu, T. Koslowski, J. Lumin. 111 (2005) 327.

[61] M. R. Shortreed, S. F. Swallen, Z.-Y. Shi, W. Tan, Z. Xu, C. Devadoss, J. S. Moore, R. Kopelman, J. Phys. Chem. B 101 (1997) 6318.

[62] O. Varnavski, I. D. W. Samuel, L.-O. Palsson, R. Beavington, P. L. Burn, T. G. III, J. Chem. Phys. 116 (2002) 8893.

[63] O. P. Varnavski, J. C. Ostrowski, L. Sukhomlinova, R. J. Twieg, G. C. Bazan, T. G. III, J. Am. Chem. Soc. 124 (2002) 1736.

[64] G. R. Fleming, G. D. Scholes, Nature 431 (2004) 256.

[65] Y. C. Cheng, R. J. Silbey, Phys. Rev. Lett. 96 (2006) 028103.

[66] M. Mohseni, P. Rebentrost, S. Lloyd, A. Aspuru-Guzik, The Journal of Chemical Physics 129 (2008) 174106.

[67] F. Caruso, A. W. Chin, A. Datta, S. F. Huelga, M. B. Plenio, The Journal of Chemical Physics 131 (2009) 105106.

[68] A. Olaya-Castro, C. F. Lee, F. F. Olsen, N. F. Johnson, Phys. Rev. B 78 (2008) 085115.

[69] H.-P. Breuer, F. Petruccione, The Theory of Open Quantum Systems, Oxford University Press, Oxford, England, 2006.

[70] D. Aharonov, A. Ambainis, J. Kempe, U. Vazirani, in: Proceedings of ACM Symposium on Theory of Computation (STOC'01), ACM Press, New York, 2001, p. 50.

[71] O. Mülken, A. Volta, A. Blumen, Phys. Rev. A 72 (2005) 042334.

[72] O. Mülken, A. Blumen, Phys. Rev. E 71 (2005) 036128.

[73] O. Mülken, A. Blumen, Phys. Rev. E 73 (2006) 066117.

[74] O. Mülken, Preprint (2007) arXiv:0710.3453

[75] I. S. Gradshteyn, I. M. Ryzhik, Table of Integrals, Series, and Products, Academic Press, 1980.

[76] S. Alexander, J. Bernasconi, W. R. Schneider, R. Orbach, Rev. Mod. Phys. 53 (1981) 175.

[77] S. Salimi, Quantum Inf. Process. 9 (2009) 75.

[78] K. Ito (Ed.), Encyclopedic Dictionary of Mathematics, MITPress, Cambridge, MA, 1987.

[79] A. Volta, O. Mülken, A. Blumen, J. Phys. A 39 (2006) 14997.
[80] X. P. Xu, J. Phys. A 42 (2009) 115205.

[81] S. Salimi, Ann. Phys. 324 (2009) 1185.

[82] O. Mülken, V. Bierbaum, A. Blumen, J. Chem. Phys. 124 (2006) 124905

[83] C. Cai, Z. Y. Chen, Macromolecules 30 (1997) 5104.

[84] R. Iwanow, D. A. May-Arrioja, D. N. Christodoulides, G. I. Stegeman, Y. Min, W. Sohler, Phys. Rev. Lett. 95 (2005) 053902.

[85] A. Bar-Haim, J. Klafter, R. Kopelman, J. Am. Chem. Soc. 119 (1997) 6197.

[86] D. Rana, G. Gangopadhyay, J. Chem. Phys. 118 (2003) 434.

[87] E. Y. Poliakov, V. Chernyak, S. Tretiak, S. Mukamel, J. Chem. Phys. 110 (1999) 8161.

[88] A. Blumen, V. Bierbaum, O. Mülken, Physica A 371 (2006) 10.

[89] A. M. Childs, E. Farhi, S. Gutmann, Quantum Information Processing 1 (2002) 35.

[90] E. Agliari, A. Blumen, O. Mülken, J. Phys. A 41 (2008) 445301.

[91] M. G. Cosenza, R. Kapral, Phys. Rev. A 46 (1992) 1850.

[92] A. Blumen, A. Jurjiu, J. Chem. Phys. 116 (2002) 2636.

[93] O. Mülken, V. Pernice, A. Blumen, Phys. Rev. E 76 (2007) 051125.

[94] S. Jespersen, I. M. Sokolov, A. Blumen, Phys. Rev. E 62 (2000) 4405.

[95] E. J. Heller, Phys. Rev. A 35 (1987) 1360.

[96] X. P. Xu, F. Liu, Phys. Lett. A 372 (2008) 6727.

[97] X. P. Xu, F. Liu, New J. Phys. 10 (2008) 123012.

[98] X. P. Xu, W. Li, F. Liu, Phys. Rev. E 78 (2008) 052103.

[99] O. Mülken, V. Pernice, A. Blumen, Phys. Rev. E 77 (2008) 021117.

[100] M. Abramowitz, I. A. Stegun (Eds.), Handbook of Mathematical Functions, Dover, New York, 1972.

[101] C. M. Bender, S. A. Orszag, Advanced Mathematical Methods for Scientists and Engineers, McGraw-Hill, Inc., 1978.

[102] X. P. Xu, Phys. Rev. E 77 (2008) 061127.

[103] O. Mülken, V. Bierbaum, A. Blumen, Phys. Rev. E 75 (2007) 031121.

[104] Y. Yin, D. E. Katsanos, S. N. Evangelou, Phys. Rev. A 77 (2008) 022302.

[105] O. Mülken, V. Pernice, A. Blumen, Phys. Rev. E 78 (2008) 021115.

[106] E. Agliari, O. Mülken, A. Blumen, Int. J. Bif. Chaos 20 (2010) 271.

[107] O. Mülken, A. Blumen, Physica E 42 (2010) 576.

[108] M. M. Sternheim, J. F. Walker, Phys. Rev. C 6 (1972) 114.

[109] P. E. Parris, Phys. Rev. B 40 (1989) 4928.

[110] X. P. Xu, Phys. Rev. E 79 (2009) On11117.

[111] A. Volta, J. Phys. A 42 (2009) 225003.

[112] A. Blumen, C. von Ferber, A. Jurjiu, T. Koslowski, Macromolecules 37 (2004) 638.

[113] W. P. Schleich, Quantum Optics in Phase Space, Wiley-VCH, Berlin, 2001.

[114] E. P. Wigner, Phys. Rev. 40 (1932) 749.

[115] M. Hillery, R. F. O'Connell, M. O. Scully, E. P. Wigner, Phys. Rep. 106 (1984) 121.

[116] W. K. Wootters, Ann. Phys. 176 (1987) 1.

[117] O. Cohendet, P. Combe, M. Sirugeu, M. Sirugue-Collin, J. Phys. A 21 (1988) 2875.

[118] U. Leonhardt, Phys. Rev. Lett. 74 (1995) 4101.

[119] U. Leonhardt, Phys. Rev. A 53 (1996) 2998.

[120] O. Mülken, A. Blumen, Phys. Rev. A 73 (2006) 012105.

[121] X. P. Xu, F. Liu, Phys. Rev. A 77 (2008) 062318.

[122] A. O. Caldeira, A. J. Legget, Ann. Phys. 149 (1983) 374. 
[123] A. O. Caldeira, A. J. Legget, Ann. Phys. 153 (1984) 445(E).

[124] S. A. Gurvitz, L. Fedichkin, D. Mozyrsky, G. P. Berman, Phys. Rev. Lett. 91 (2003) 066801.

[125] D. Solenov, L. Fedichkin, Phys. Rev. A 73 (2006) 012308.

[126] D. Solenov, L. Fedichkin, Phys. Rev. A 73 (2006) 012313.

[127] L. Fedichkin, D. Solenov, C. Tamon, Q. Inf. Processing 6 (2006) 263.

[128] S. Salimi, R. Radgohar, J. Phys. A 42 (2009) 475302.
[129] O. Mülken, L. Mühlbacher, T. Schmid, A. Blumen, Phys. Rev. E 81 (2010) 041114.

[130] L. Mühlbacher, J. Ankerhold, C. Escher, J. Chem. Phys. 121 (2004) 12696.

[131] L. Mühlbacher, J. Ankerhold, J. Chem. Phys. 122 (2005) 184715. 